\title{
APERTURE EFFECTS ON THE OXYGEN ABUNDANCE DETERMINATIONS FROM CALIFA DATA
}

\author{
J. Iglesias-Páramo ${ }^{1,2}$, J. M. Vílchez ${ }^{1}$, F. F. Rosales-Ortega ${ }^{3}$, S. F. Sánchez ${ }^{4}$, S. Duarte Puertas ${ }^{1}$, V. Petropoulou ${ }^{5}$, \\ A. Gil de Paz ${ }^{6}$, L. Galbany ${ }^{7,8}$, M. Mollá ${ }^{9}$, C. Catalán-Torrecilla ${ }^{6}$, A. Castillo Morales ${ }^{6}$, D. Mast ${ }^{10,11}$, B. Husemann ${ }^{12}$, \\ R. García-Benito ${ }^{1}$, M. A. Mendoza ${ }^{1}$, C. Kehrig $^{1}$, E. Pérez-Montero ${ }^{1}$, P. Papaderos ${ }^{13}$, J. M. Gomes ${ }^{13}$, C. J. Walcher ${ }^{14}$, \\ R. M. González Delgado ${ }^{1}$, R. A. Marino ${ }^{6,15}$, Á. R. López-SÁnchez ${ }^{16,17}$, B. Ziegler ${ }^{18}$, H. Flores $^{19}$, And J. Alves $^{18}$ \\ ${ }^{1}$ Instituto de Astrofísica de Andalucía-CSIC, Glorieta de la Astronomía s.n., E-18008 Granada, Spain; jiglesia@iaa.es \\ ${ }^{2}$ Estación Experimental de Zonas Áridas-CSIC, Ctra. de Sacramento s.n., La Cañada, Almería, Spain \\ ${ }^{3}$ Instituto Nacional de Astrofísica, Óptica y Electrónica, Luis E. Erro 1, 72840 Tonantzintla, Puebla, Mexico \\ ${ }^{4}$ Instituto de Astronomía, Universidad Nacional Autónoma de Méjico, A.P. 70-264, 04510 México, D.F., Mexico \\ 5 INAF-OA Brera, via Brera 28, I-20121 Milano, Italy \\ ${ }^{6}$ Departamento de Astrofísica y CC. de la Atmósfera, Universidad Complutense de Madrid, E-28040, Madrid, Spain \\ ${ }^{7}$ Millennium Institute of Astrophysics, Chile \\ ${ }^{8}$ Departamento de Astronomía, Universidad de Chile, Camino El Observatorio 1515, Las Condes, Santiago, Chile \\ ${ }^{9}$ Departamento de Investigación Básica, CIEMAT, Avda. Complutense 40, E-28040 Madrid, Spain \\ ${ }^{10}$ Observatorio Astronómico, Laprida 854, X5000BGR, Córdoba, Argentina \\ ${ }^{11}$ Consejo de Investigaciones Científicas y Técnicas de la República Argentina, Avda. Rivadavia 1917, C1033AAJ, CABA, Argentina \\ ${ }^{12}$ European Southern Observatory (ESO), Karl-Schwarzschild-Str.2, D-85748 Garching b. München, Germany \\ ${ }^{13}$ Instituto de Astrofísica e Ciências do Espaço, Universidade do Porto, CAUP, Rua das Estrelas, P-4150-762 Porto, Portugal \\ ${ }^{14}$ Leibniz-Institude für Astrophysik Potsdam (AIP), An der Sternwarte 16, D-14482 Potsdam, Germany \\ ${ }_{16}^{15}$ Department of Physics, Institute for Astronomy, ETH Zürich, CH-8093 Zürich, Switzerland \\ ${ }^{16}$ Australian Astronomical Observatory, P.O. Box 915, North Ryde, NSW 1670, Australia \\ ${ }^{17}$ Department of Physics and Astronomy, Macquarie University, NSW 2109, Australia \\ ${ }^{18}$ University of Vienna, Türkenschanzstrasse 17, A-1180 Vienna, Austria \\ ${ }^{19}$ GEPI, Observatoire de Paris, CNRS, 5 Place Jules Janssen, Meudon F-92195, France \\ Received 2015 December 17; revised 2016 May 2; accepted 2016 May 2; published 2016 July 22
}

\begin{abstract}
This paper aims to provide aperture corrections for emission lines in a sample of spiral galaxies from the Calar Alto Legacy Integral Field Area Survey (CALIFA) database. In particular, we explore the behavior of the $\log ([\mathrm{O}$ III $] \lambda 5007 /$ $\mathrm{H} \beta) /([\mathrm{N} \mathrm{II}] \lambda 6583 / \mathrm{H} \alpha)(\mathrm{O} 3 \mathrm{~N} 2)$ and $\log [\mathrm{N} \mathrm{II}] \lambda 6583 / \mathrm{H} \alpha(\mathrm{N} 2)$ flux ratios since they are closely connected to different empirical calibrations of the oxygen abundances in star-forming galaxies. We compute the median growth curves of $\mathrm{H} \alpha, \mathrm{H} \alpha / \mathrm{H} \beta, \mathrm{O} 3 \mathrm{~N} 2$, and $\mathrm{N} 2$ up to $2.5 R_{50}$ and 1.5 disk $R_{\text {eff }}$. These distances cover most of the optical spatial extent of the CALIFA galaxies. The growth curves simulate the effect of observing galaxies through apertures of varying radii. We split these growth curves by morphological types and stellar masses to check if there is any dependence on these properties. The median growth curve of the $\mathrm{H} \alpha$ flux shows a monotonous increase with radius with no strong dependence on galaxy inclination, morphological type, and stellar mass. The median growth curve of the $\mathrm{H} \alpha / \mathrm{H} \beta$ ratio monotonically decreases from the center toward larger radii, showing for small apertures a maximum value of $\approx 10 \%$ larger than the integrated one. It does not show any dependence on inclination, morphological type, and stellar mass. The median growth curve of $\mathrm{N} 2$ shows a similar behavior, decreasing from the center toward larger radii. No strong dependence is seen on the inclination, morphological type, and stellar mass. Finally, the median growth curve of O3N2 increases monotonically with radius, and it does not show dependence on the inclination. However, at small radii it shows systematically higher values for galaxies of earlier morphological types and for high stellar mass galaxies. Applying our aperture corrections to a sample of galaxies from the SDSS survey at $0.02 \leqslant z \leqslant 0.3$ shows that the average difference between fiber-based and aperture-corrected oxygen abundances, for different galaxy stellar mass and redshift ranges, reaches typically to $\approx 11 \%$, depending on the abundance calibration used. This average difference is found to be systematically biased, though still within the typical uncertainties of oxygen abundances derived from empirical calibrations. Caution must be exercised when using observations of galaxies for small radii (e.g., below 0.5 $R_{\text {eff }}$ ) given the high dispersion shown around the median growth curves. Thus, the application of these median aperture corrections to derive abundances for individual galaxies is not recommended when their fluxes come from radii much smaller than either $R_{50}$ or $R_{\text {eff }}$.
\end{abstract}

Key words: galaxies: abundances - galaxies: general - galaxies: ISM

Supporting material: machine-readable tables

\section{INTRODUCTION}

Bidimensional spectroscopy is becoming increasingly important as a powerful observational technique capable of providing new results on the properties of galaxies. Many instruments (commonly known as Integral Field Spectrographs (IFS)) have been developed in the last few years to produce bidimensional spectroscopy, most of them based on arrays of fibers that collect light from the sky area of interest and drive it through a dispersor. The main limitation of these fiber-fed spectrograps is their limited field of view, which makes impossible the blind observation of large areas of the sky to observe large amounts of galaxies as is the case of the large-scale surveys like SDSS (York et al. 2000), 2dFGRS (Colless et al. 2001), VVDS (Le Fèvre et al. 2005), z-COSMOS (Lilly et al. 2007), and DEEP/DEEP2 (Davis et al. 2003). 

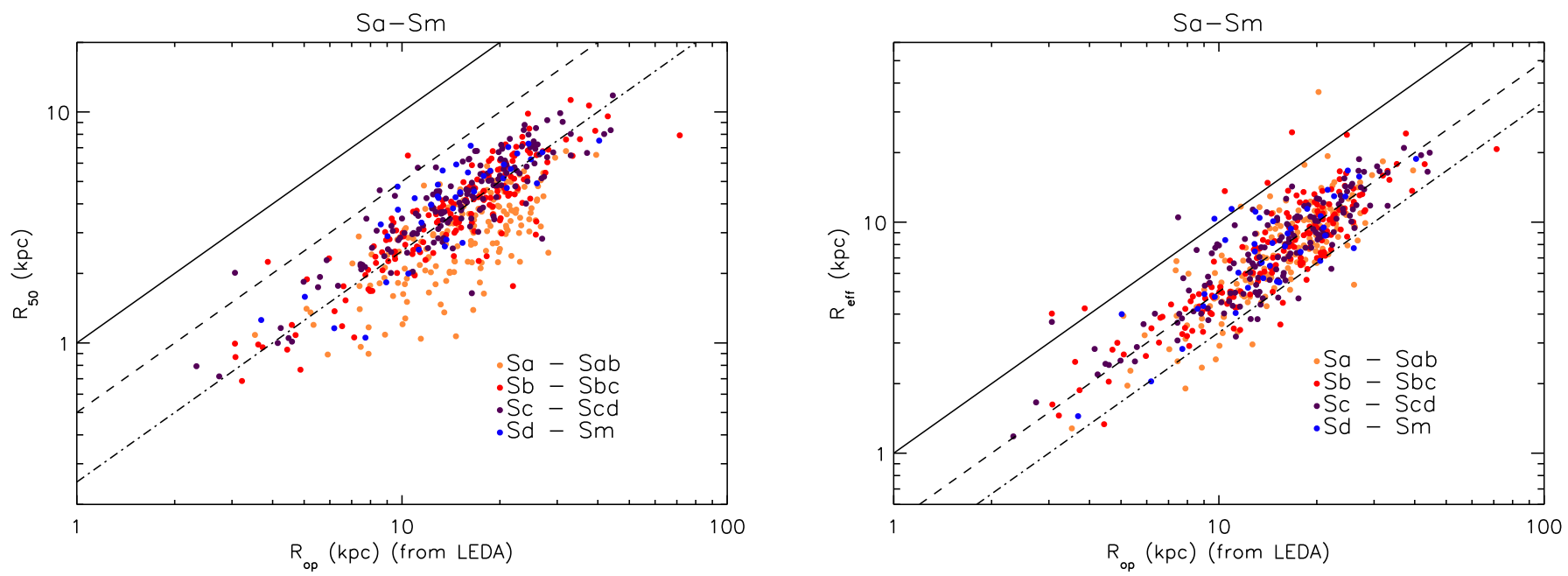

Figure 1. Left: $R_{\mathrm{op}}$ vs. $R_{50}$ for the spiral galaxies of the CALIFA sample. Solid, dashed, and dot-dashed lines correspond to $R_{\mathrm{op}}=R_{50}, R_{\mathrm{op}}=2 \times R_{50}$ and $R_{\mathrm{op}}=4 \times R_{50}$, respectively. Right: $R_{\mathrm{op}}$ vs. $R_{\mathrm{eff}}$ for the spiral galaxies of the CALIFA sample. Solid, dashed, and dot-dashed lines correspond to $R_{\mathrm{op}}=R_{\mathrm{eff}}$, $R_{\mathrm{op}}=2 \times R_{\mathrm{eff}}$, and $R_{\mathrm{op}}=3 \times R_{\mathrm{eff}}$ respectively.
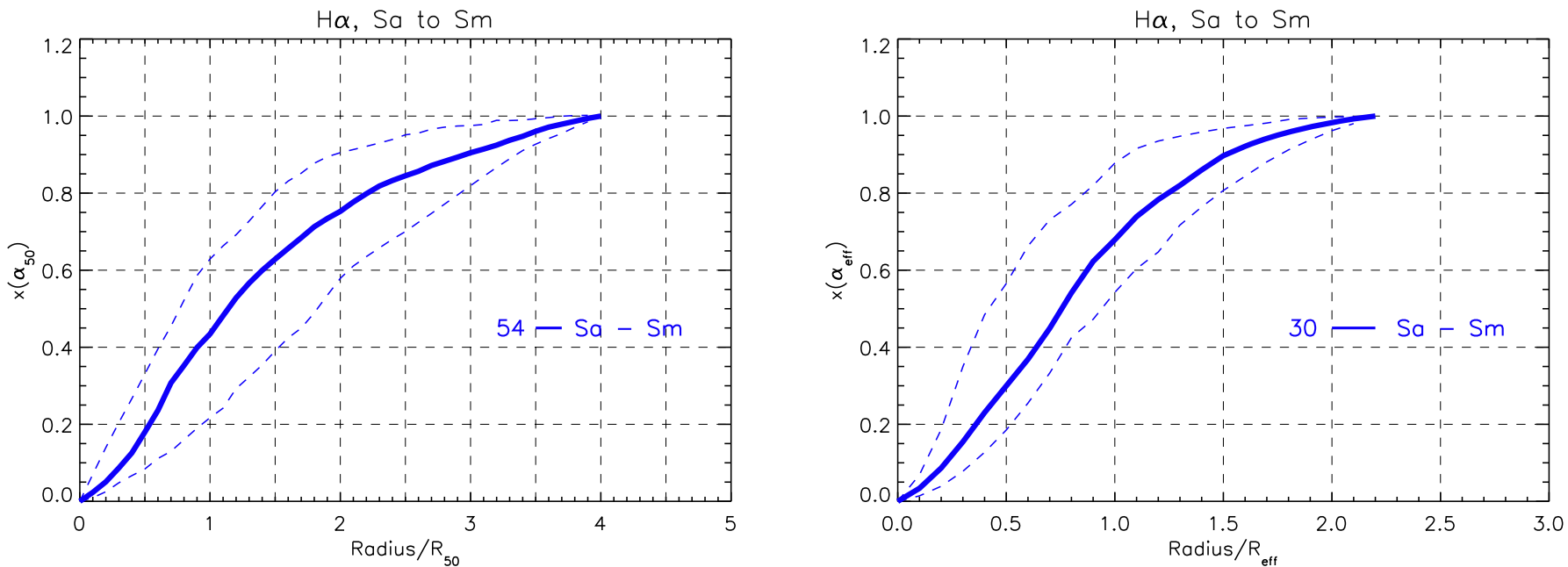

Figure 2. Left: $x\left(\alpha_{50}\right)$ for CALIFA spiral galaxies with $R_{50}<9^{\prime \prime}$ normalized to $4 R_{50}$. Solid lines correspond to median values. Dashed lines contain $68.2 \%$ of the values. Right: $x$ ( $\left.\alpha_{\text {eff }}\right)$ for CALIFA spiral galaxies with $R_{\text {eff }}<16$ ". 4 normalized to $2.2 R_{\text {eff }}$.

The best option for IFS surveys is thus to focus on selected individual objects, but this is very time consuming and prevents the study of large samples of galaxies. So far, there are only a few surveys employing IFS, among which we highlight SAURON (Bacon et al. 2001), ATLAS-3D (Cappellari et al. 2011), PINGS (Rosales-Ortega et al. 2010), VENGA (Blanc et al. 2010), VIXENS (Heiderman et al. 2011), and Calar Alto Legacy Integral Field Area Survey (CALIFA) (Sánchez et al. 2012).

The CALIFA Survey (Sánchez et al. 2012) is observing a statistically well-defined sample of 600 galaxies in the local universe with the Potsdam Multi Aperture Spectrograph in the PPAK mode (Roth et al. 2005) at the $3.5 \mathrm{~m}$ telescope at Calar Alto Observatory. The survey benefits from the wide field of view of PPAK (about $1 \mathrm{arcmin}^{2}$ ) compared to similar instruments at other telescopes. Thus, CALIFA galaxies are mapped over most of their optical spatial extent, and so far it has allowed upto-date complete bidimensional studies of galaxy properties like star formation histories (Cid Fernandes et al. 2013, González
Table 1

Description of the Sizes of the Two Subsamples after Each of the Filters Imposed

\begin{tabular}{llll}
\hline \hline \multicolumn{1}{c}{$S_{50}$} & & \multicolumn{1}{c}{$S_{\text {eff }}$} \\
\hline Initial sample & 402 & Initial sample & $394^{\mathrm{a}}$ \\
Excluding Es and S0s & 301 & Excluding Es and S0s & 296 \\
Excluding AGNs & 212 & Excluding AGNs & 208 \\
$R_{50} \leqslant 14 " .4$ & 171 & $R_{\text {eff }} \leqslant 24^{\prime \prime}$ & 138 \\
$f(\mathrm{H} \alpha) \geqslant 0, \sigma(\mathrm{H} \alpha) / f(\mathrm{H} \alpha)$ & 165 & $f(\mathrm{H} \alpha) \geqslant 0, \sigma(\mathrm{H} \alpha) / f(\mathrm{H} \alpha)$ & 133 \\
$\leqslant 0.333$ & & $\leqslant 0.333$ & \\
\hline
\end{tabular}

Note.

${ }^{\text {a }}$ The initial number of galaxies in $S_{\text {eff }}$ is different than that in $S_{50}$ because $R_{\text {eff }}$ could not be determined for eight galaxies, and thus they are excluded from the analysis.

Delgado et al. 2014), the properties of the ionized gas in earlytype galaxies (Kehrig et al. 2012; Papaderos et al. 2013; Singh 
et al. 2013; Gomes et al. 2015a), the properties of large samples of H II regions (Sánchez et al. 2013, 2014), or the effects of spatial resolution at different redshifts (Mast et al. 2014) among others.

In addition to this, the CALIFA database allows the study of the biases introduced when galaxies are observed through small and size-limited apertures, which usually are single-fiber spectrographs. Several studies already have noticed the existence of such aperture effects in the properties of early- and late-type galaxies

Table 2

Basic Properties of the Samples $S_{50}$ and $S_{\text {eff }}$ : Median Values of the Stellar Masses, $R_{50}$ and $R_{\text {eff }}$; Number of Galaxies Split into Two Bins of Morphological Types

\begin{tabular}{lcc}
\hline \hline & $S_{50}$ & $S_{\text {eff }}$ \\
\hline$\left\langle\log M^{*} / M_{\odot}\right\rangle$ & 10.25 & 10.25 \\
$\left\langle R_{50}\right\rangle\left(^{\prime \prime}\right)$ & 11.04 & $\ldots$ \\
$\left\langle R_{\text {eff }}\right\rangle\left(^{\prime \prime}\right)$ & $\ldots$ & 20.31 \\
$\mathrm{Sa}+\mathrm{Sab}+\mathrm{Sb}+\mathrm{Sbc}$ & 107 & 85 \\
$\mathrm{Sc}+\mathrm{Scd}+\mathrm{Sd}+\mathrm{Sdm}+\mathrm{Sm}$ & 85 & 48 \\
\hline
\end{tabular}

Table 3

Comparison of the H $\alpha$ Growth Curve and Average Dispersion of IP13 with the Ones Obtained in this Work for all the Spiral Galaxies Disregarding the Stellar Mass, Morphological Types, and Inclination

\begin{tabular}{lcccc}
\hline \hline$r / R_{50}$ & $x\left(\alpha_{50}\right)$ & \multicolumn{1}{c}{$\sigma\left(x\left(\alpha_{50}\right)\right)$} & $x\left(\alpha_{50}\right)$ & $\begin{array}{c}\sigma\left(x\left(\alpha_{50}\right)\right) \\
\text { (This Work) }\end{array}$ \\
\hline 0.3 & 0.091 & 0.074 & 0.085 & 0.060 \\
0.5 & 0.194 & 0.119 & 0.182 & 0.102 \\
0.7 & 0.301 & 0.156 & 0.283 & 0.126 \\
0.9 & 0.419 & 0.158 & 0.397 & 0.132 \\
1.1 & 0.530 & 0.146 & 0.506 & 0.134 \\
1.3 & 0.629 & 0.130 & 0.620 & 0.121 \\
1.5 & 0.718 & 0.117 & 0.715 & 0.121 \\
1.7 & 0.798 & 0.101 & 0.801 & 0.104 \\
1.9 & 0.854 & 0.079 & 0.865 & 0.078 \\
2.1 & 0.917 & 0.070 & 0.920 & 0.050 \\
2.3 & 0.961 & 0.039 & 0.965 & 0.027 \\
\hline
\end{tabular}

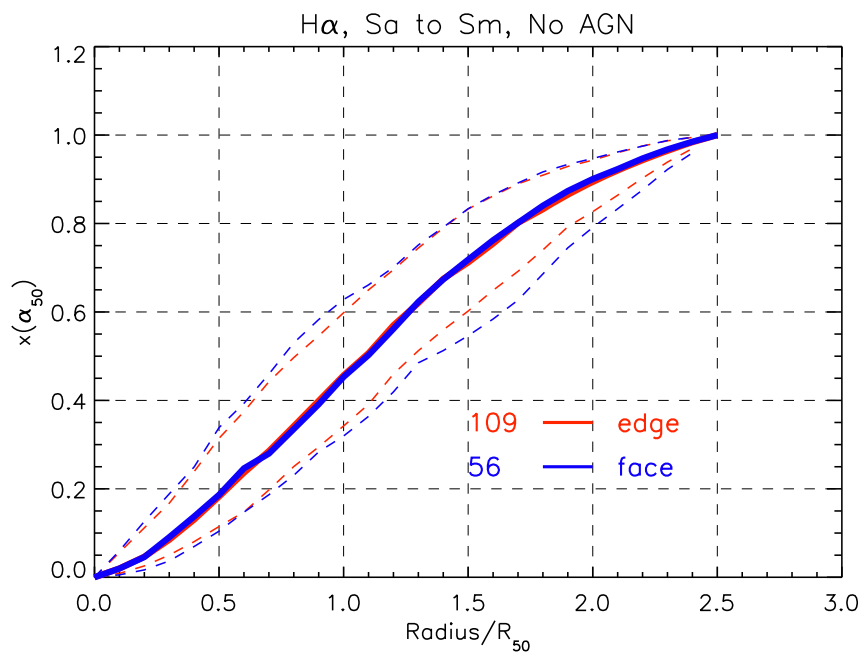

(e.g., Kehrig et al. 2013; Gomes et al. 2015b). Aperture effects on galaxy properties have been previously addressed by the following different approaches (e.g., Hopkins et al. 2003; Brinchmann et al. 2004; Ellis et al. 2005; Kewley et al. 2005; Salim et al. 2007; Gerssen et al. 2012; Zahid et al. 2013). On top of that, a recent study by Richards et al. (2015) based on part of the SAMI Galaxy Survey (Allen et al. 2015; Bryant et al. 2015; Sharp et al. 2015) concludes that biases in the estimation of the total instantaneous star formation rate (SFR) of a galaxy arise when the aperture correction is built only from spectra of the nuclear region of galaxies.

A preliminary study of the aperture effects based on CALIFA data was presented in Iglesias-Páramo et al. (2013, IP13) and was devoted to the $\mathrm{H} \alpha$ and $\mathrm{H} \beta$ emission-line fluxes. In the present work we extend their analysis using a much larger sample of galaxies, which allows us to perform a more detailed study. We try to go beyond and focus on observational parameters related to the derivation of the oxygen abundances, like the (widely used in the literature) $\mathrm{N} 2$ and $\mathrm{O} 3 \mathrm{~N} 2$ parameters.

This is a crucial point since spiral galaxies are known to show radial abundance gradients, which means that observing them through a reduced aperture does not necessarily provide complete information on the spatial abundance distribution. Regarding this point, it has been reported in the literature that the oxygen abundance derived for the integrated fluxes of emission lines of spiral galaxies equals the corresponding abundance of their $\mathrm{H}_{\text {II }}$ regions at a typical galactocentric distance of $0.4 \times R_{\text {opt }}$ (Pilyugin et al. 2004, Moustakas \& Kennicutt 2006). This result, linking the abundances of $\mathrm{H}$ II regions at a fixed galactocentric radius and the abundance obtained for the integrated emission-line flux ratios from the whole disk of spiral galaxies, gives some support for adopting a reference characteristic value for the abundance of a spiral galaxy. Nonetheless, we should bear in mind that the integrated emission of the spiral disks represents in fact a composite spectrum, including different $\mathrm{H}$ II regions (plus diffuse ionized ISM) with varying physical conditions and chemical compositions.

Although there is a classical method to derive oxygen abundances of bright star-forming regions based on atomic data and the fluxes of emision lines ([O II $] \lambda 3727 \AA$, [O III] $] \lambda 4363 \AA$, $[\mathrm{O}$ III] $\lambda 5007 \AA$ ), known as the direct method, it cannot be used for more distant or intrinsically fainter galaxies since some of

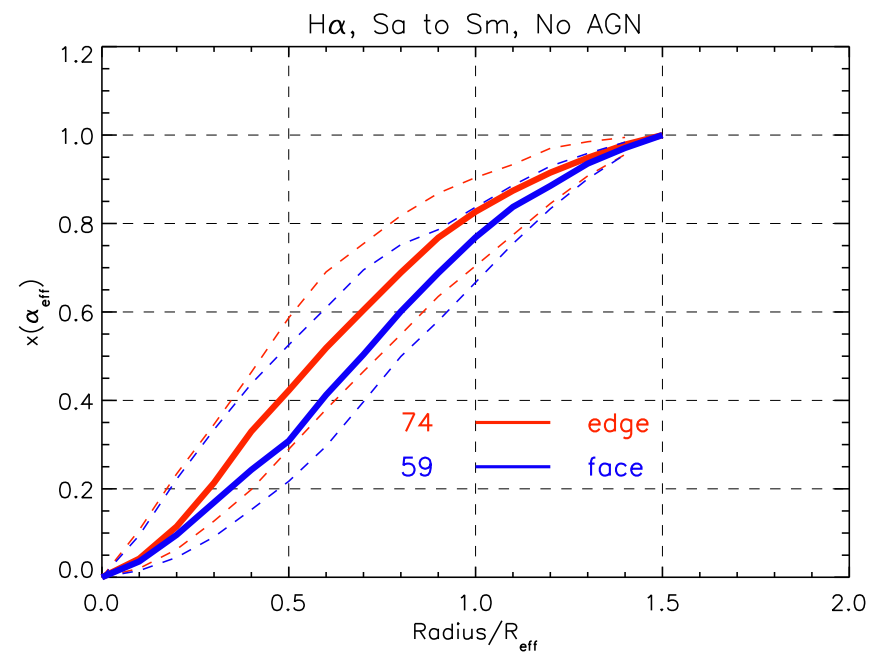

Figure 3. $x\left(\alpha_{50}\right)$ (left) and $x\left(\alpha_{\text {eff }}\right)$ (right) for galaxies with different inclination. Solid lines correspond to median values. Dashed lines contain $68.2 \%$ of the distribution. Face (blue) and edge (red) galaxies have $b / a>0.4$ and $b / a \leqslant 0.4$, respectively. The numbers within the plot box indicate the sizes of the samples. 

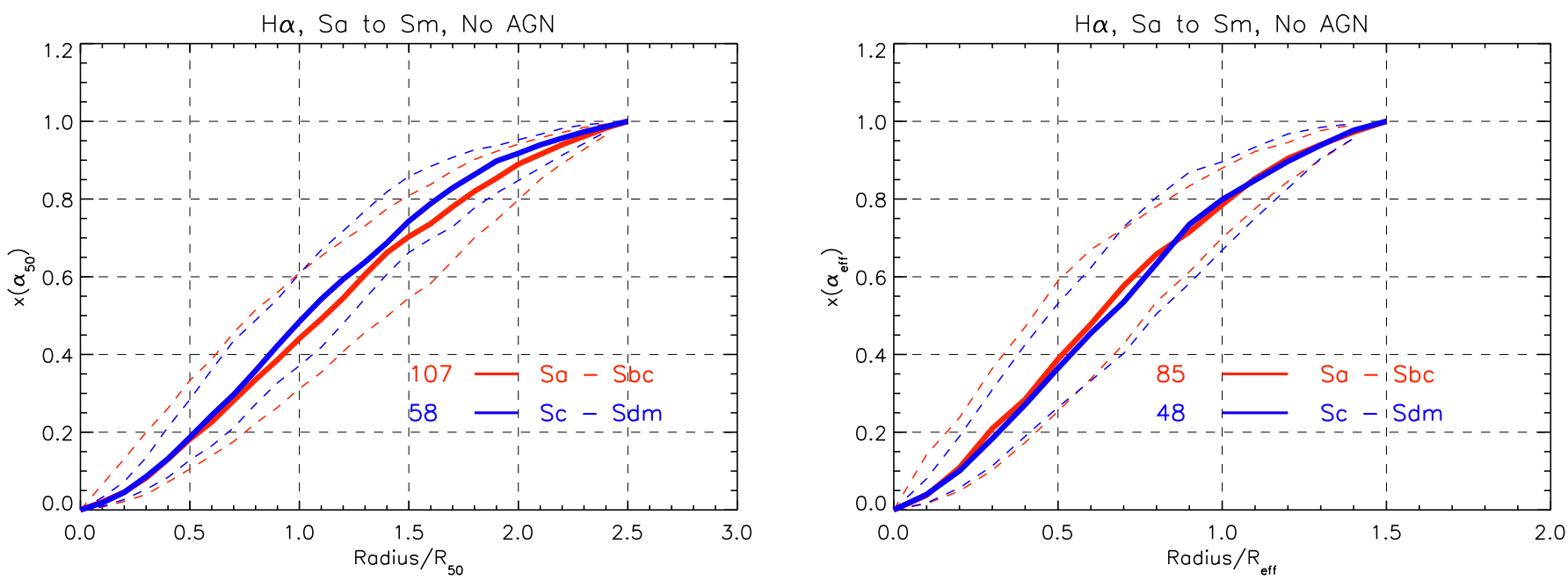

Figure 4. $x\left(\alpha_{50}\right)$ (left) and $x\left(\alpha_{\text {eff }}\right)$ (right) for galaxies with different morphological types. Solid lines correspond to median values. Dashed lines contain $68.2 \%$ of the distribution. Sa-Sbc and Sc-Sm galaxies are represented in blue and red, respectively. The numbers within the plot box indicate the sizes of the samples.
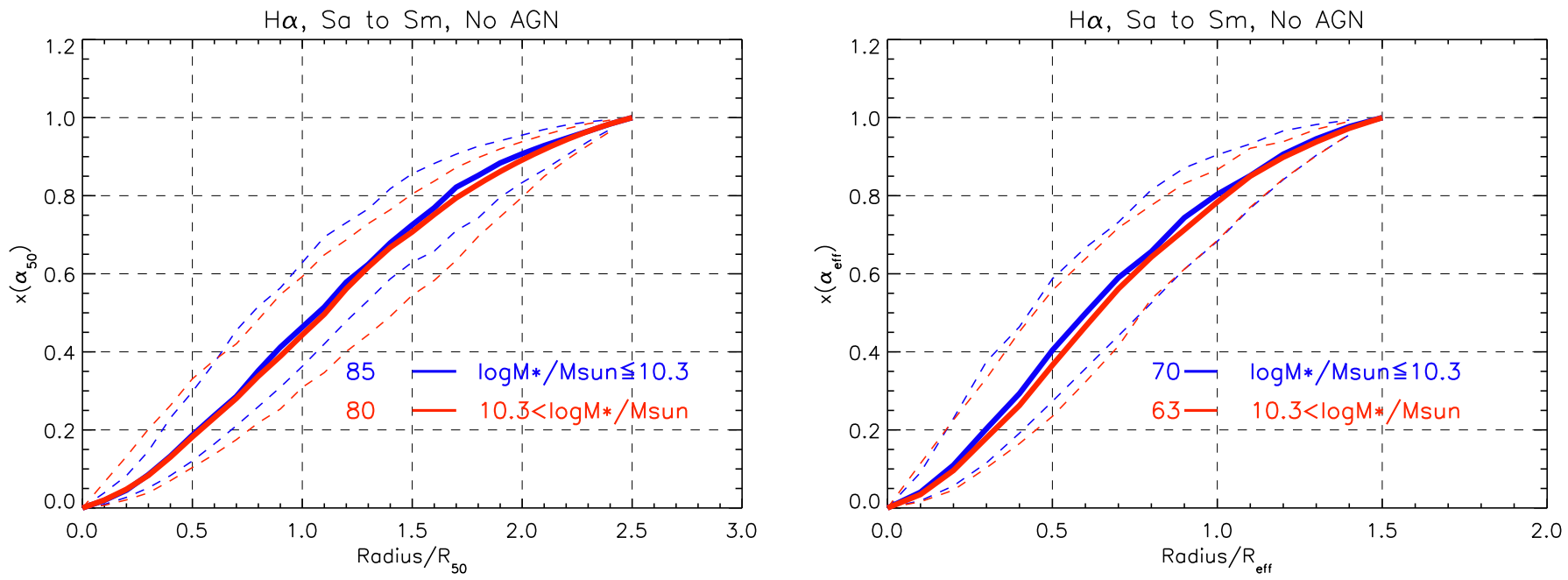

Figure 5. $x\left(\alpha_{50}\right)$ (left) and $x\left(\alpha_{\text {eff }}\right)$ (right) for galaxies with different stellar masses. Solid lines correspond to median values. Dashed lines contain $68.2 \%$ of the distribution. Galaxies with $\log M^{*} / M_{\odot} \leqslant 10.3$ and $\log M^{*} / M_{\odot}>10.3$ are represented in blue and red, respectively. The numbers within the plot box indicate the sizes of the samples.
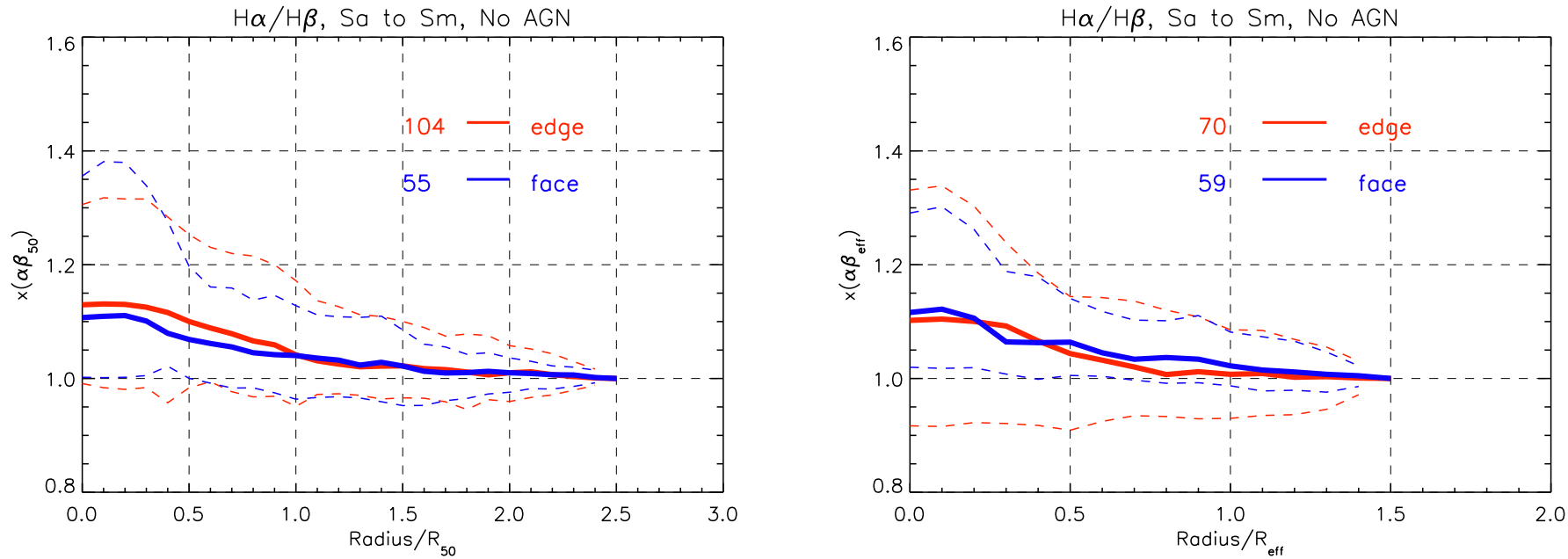

Figure 6. $x\left(\alpha \beta_{50}\right)$ (left) and $x\left(\alpha \beta_{\text {eff }}\right)$ (right) for galaxies with different inclination. Solid lines correspond to median values. Dashed lines contain $68.2 \%$ of the distribution. Face (blue) and edge (red) galaxies have $b / a>0.4$ and $b / a \leqslant 0.4$, respectively. The numbers within the plot box indicate the sizes of the samples. 

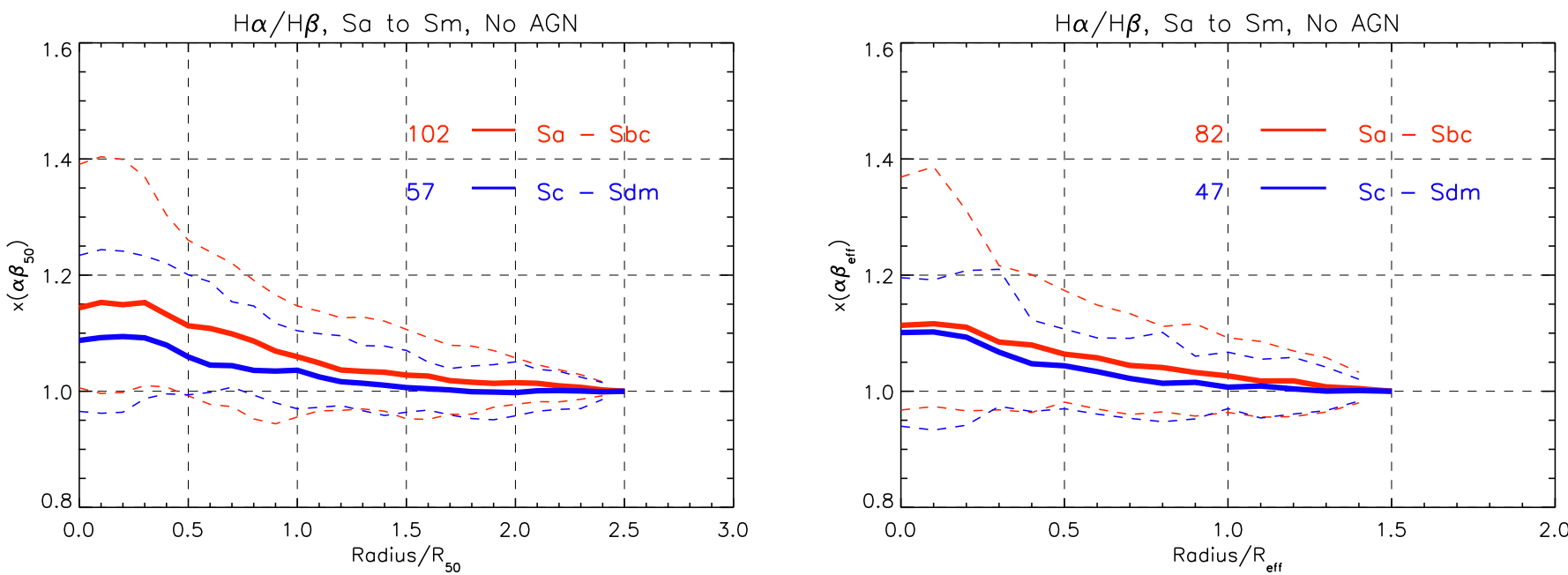

Figure 7. $x\left(\alpha \beta_{50}\right)$ (left) and $x\left(\alpha \beta_{\text {eff }}\right)$ (right) for galaxies with different morphological types. Solid lines correspond to median values. Dashed lines contain $68.2 \%$ of the distribution. Sa-Sbc and Sc-Sm galaxies are represented in blue and red, respectively. The numbers within the plot box indicate the sizes of the samples.
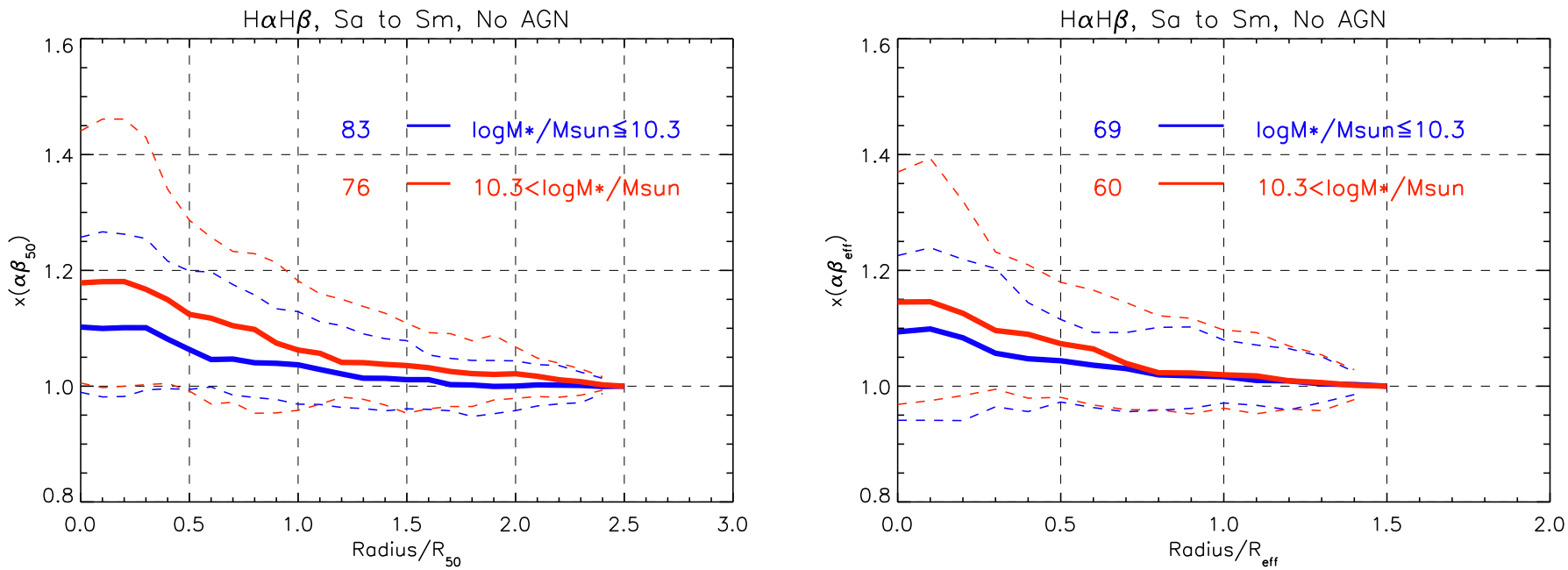

Figure 8. $x\left(\alpha \beta_{50}\right)$ (left) and $x\left(\alpha \beta_{\text {eff }}\right)$ (right) for galaxies with different stellar masses. Solid lines correspond to median values. Dashed lines contain $68.2 \%$ of the distribution. Galaxies with $\log M^{*} / M_{\odot} \leqslant 10.3$ and $\log M^{*} / M_{\odot}>10.3$ are represented in blue and red, respectively. The numbers within the plot box indicate the sizes of the samples.
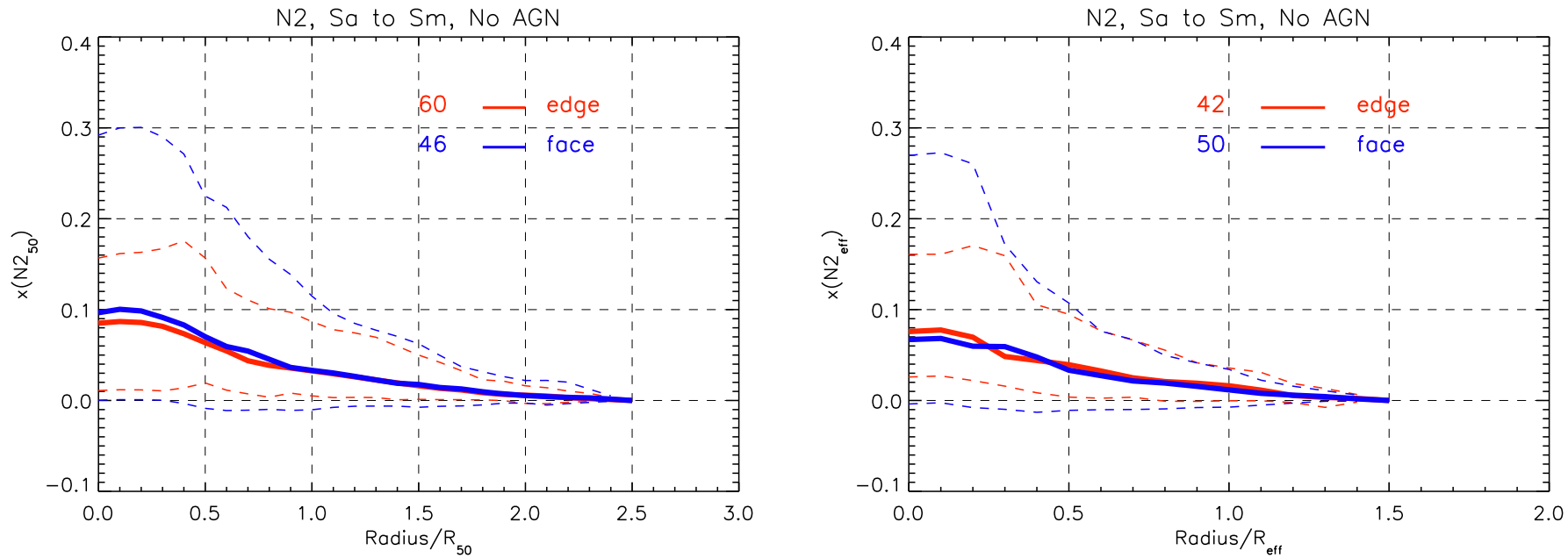

Figure 9. $x\left(\mathrm{~N} 2_{50}\right)$ (left) and $x\left(\mathrm{~N} 2_{\text {eff }}\right)$ (right) for galaxies with different inclination. Solid lines correspond to median values. Dashed lines contain $68.2 \%$ of the distribution. Face (blue) and edge (red) galaxies have $b / a>0.4$ and $b / a \leqslant 0.4$ respectively. The numbers within the plot box indicate the sizes of the samples. 

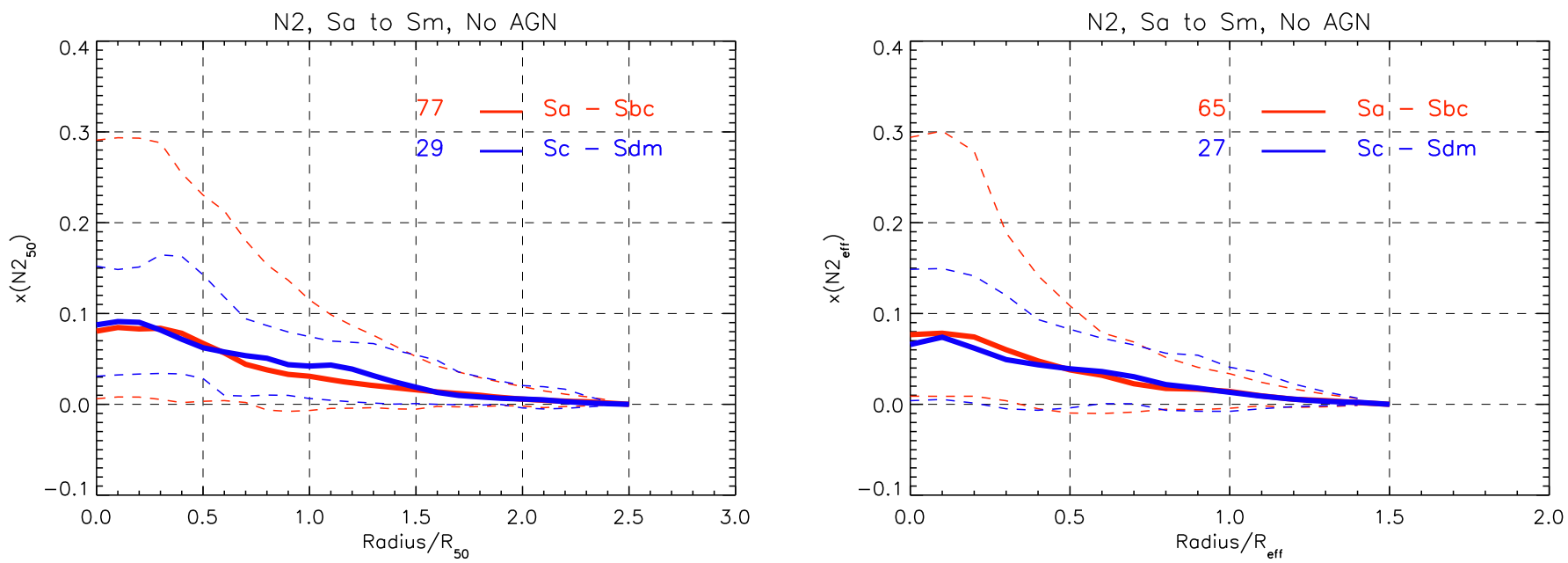

Figure 10. $x\left(\mathrm{~N} 2_{50}\right)$ (left) and $x\left(\mathrm{~N} 2_{\text {eff }}\right)$ (right) for galaxies with different morphological types. Solid lines correspond to median values. Dashed lines contain $68.2 \%$ of the distribution. Sa-Sbc and Sc-Sm galaxies are represented in blue and red, respectively. The numbers within the plot box indicate the sizes of the samples.
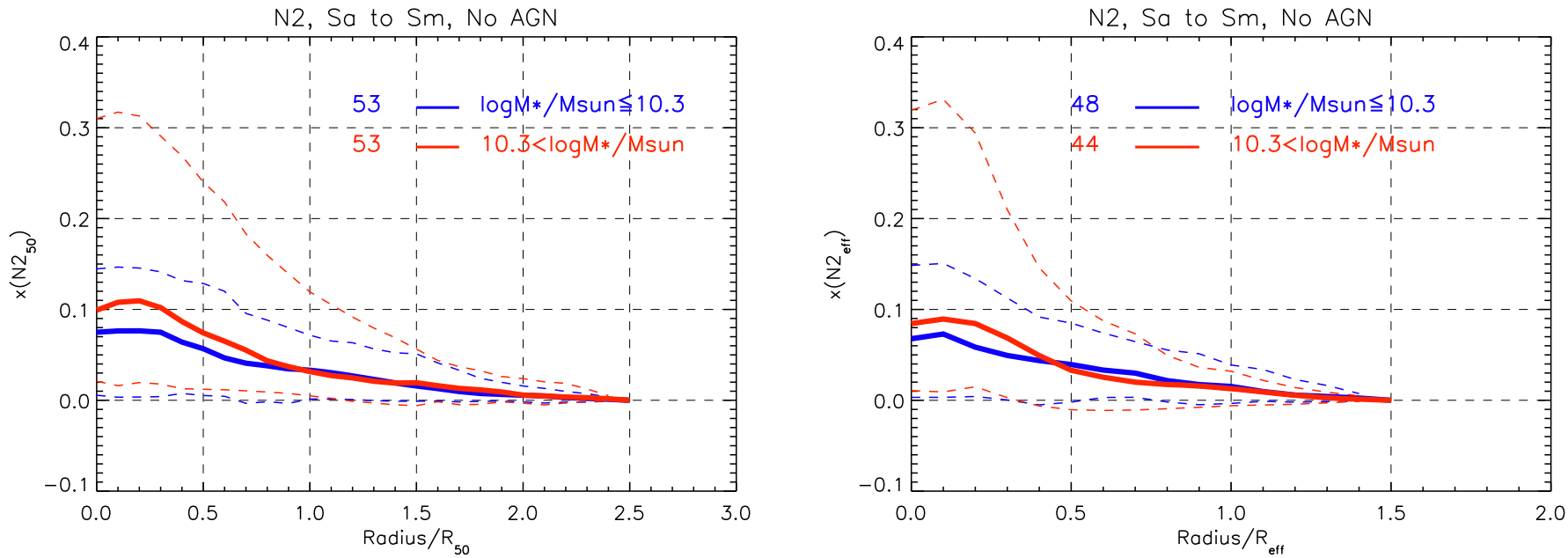

Figure 11. $x\left(\mathrm{~N}_{50}\right)$ (left) and $x\left(\mathrm{~N} 2_{\text {eff }}\right)$ (right) for galaxies with different stellar masses. Solid lines correspond to median values. Dashed lines contain $68.2 \%$ of the distribution. Galaxies with $\log M^{*} / M_{\odot} \leqslant 10.3$ and $\log M^{*} / M_{\odot}>10.3$ are represented in blue and red, respectively. The numbers within the plot box indicate the sizes of the samples.
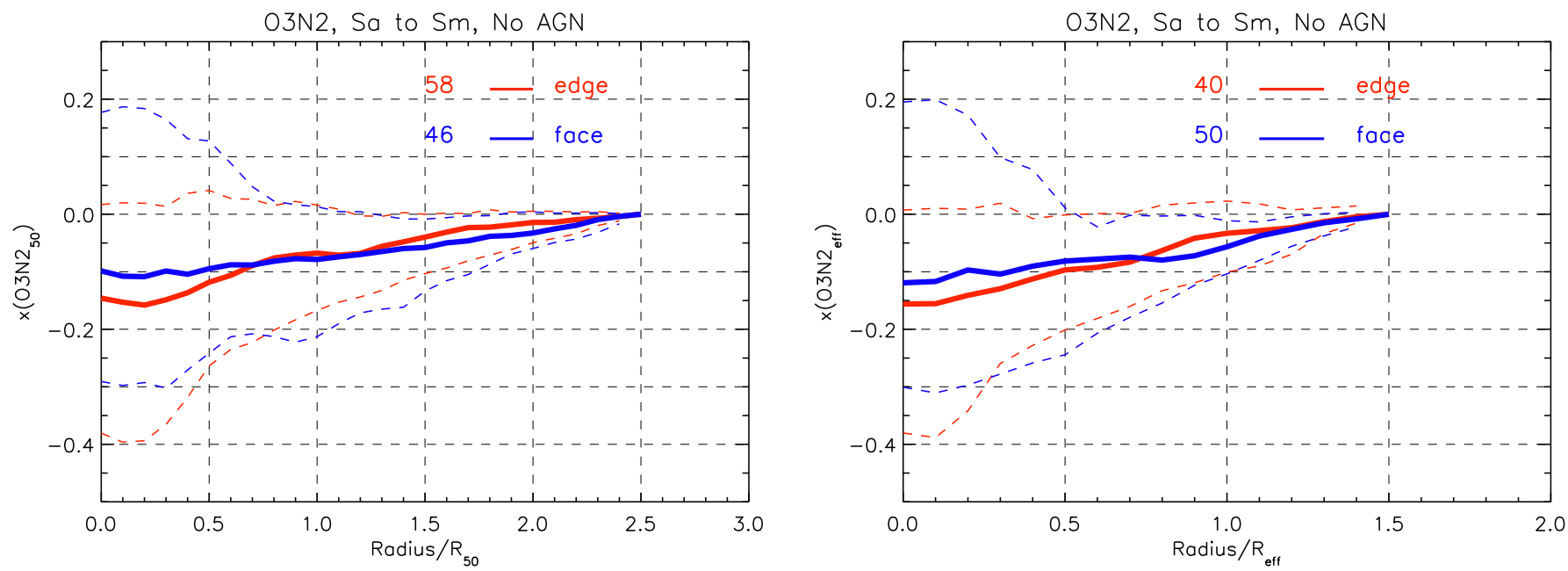

Figure 12. $x\left(\mathrm{O} 3 \mathrm{~N} 2_{50}\right)$ (left) and $x\left(\mathrm{O} 3 \mathrm{~N} 2_{\text {eff }}\right)$ (right) for galaxies with different inclination. Solid lines correspond to median values. Dashed lines contain $68.2 \%$ of the distribution. Face (blue) and edge (red) galaxies have $b / a>0.4$ and $b / a \leqslant 0.4$, respectively. The numbers within the plot box indicate the sizes of the samples. 

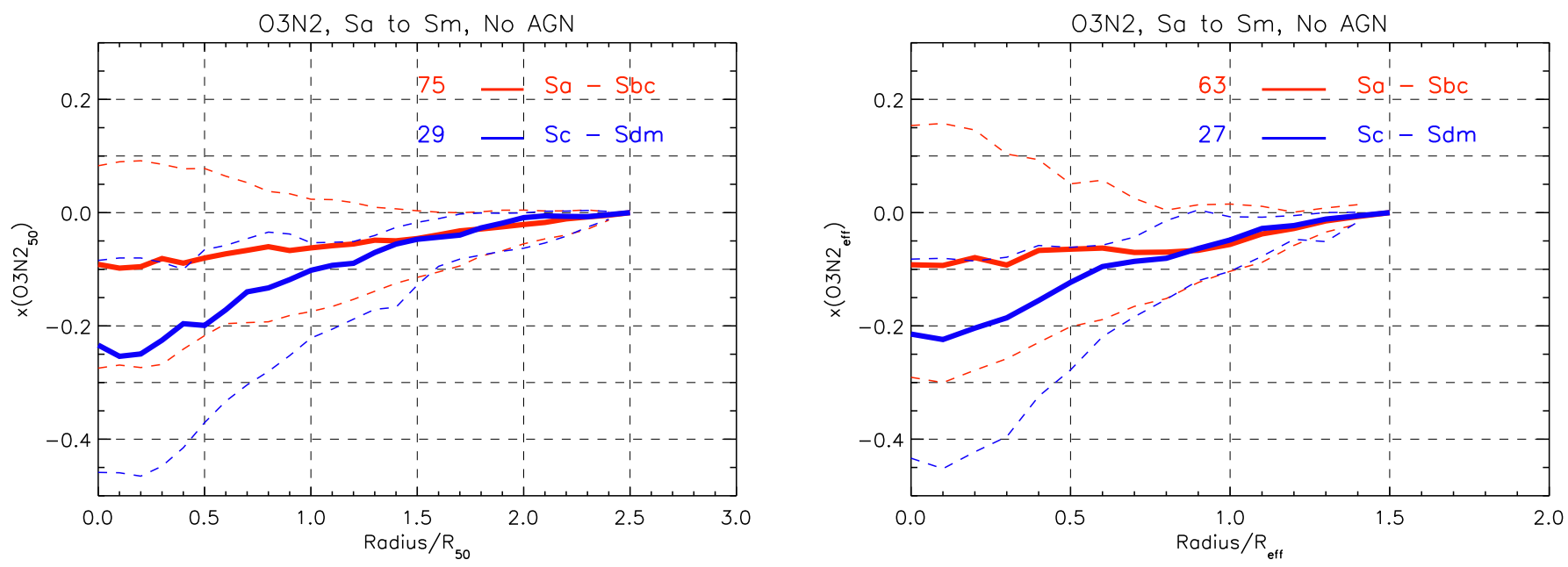

Figure 13. $x\left(\mathrm{O} 3 \mathrm{~N} 2_{50}\right)$ (left) and $x\left(\mathrm{O} 3 \mathrm{~N} 2_{\text {eff }}\right)$ (right) for galaxies with different morphological types. Solid lines correspond to median values. Dashed lines contain $68.2 \%$ of the distribution. Sa-Sbc and Sc-Sm galaxies are represented in blue and red, respectively. The numbers within the plot box indicate the sizes of the samples.
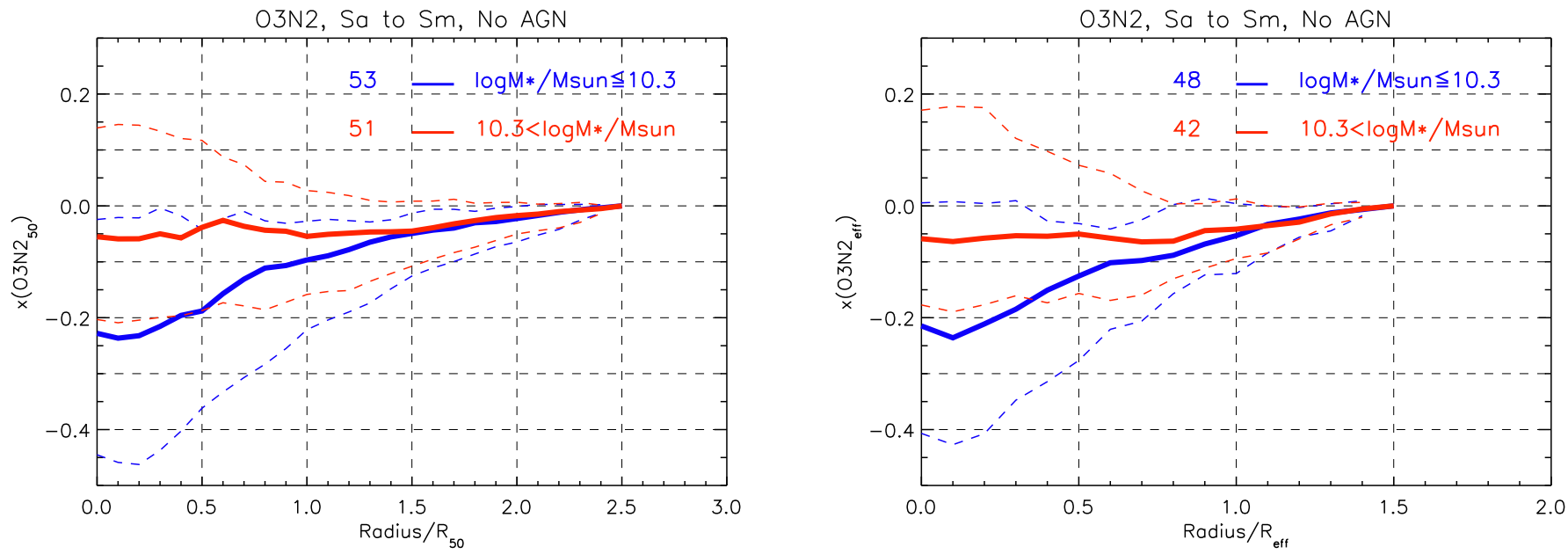

Figure 14. $x\left(\mathrm{O} 3 \mathrm{~N} 2_{50}\right)$ (left) and $x\left(\mathrm{O} 3 \mathrm{~N} 2_{\text {eff }}\right)$ (right) for galaxies with different stellar masses. Solid lines correspond to median values. Dashed lines contain $68.2 \%$ of the distribution. Galaxies with $\log M^{*} / M_{\odot} \leqslant 10.3$ and $\log M^{*} / M_{\odot}>10.3$ are represented in blue and red, respectively. The numbers within the plot box indicate the sizes of the samples.

these lines are usually not detected. For this reason, abundance calibrations based on theoretical or empirical methods based only on the brightest emission lines must be used. Significant differences among the different calibrations commonly used in the literature (see Kewley \& Ellison 2008 for an extensive discussion on this topic) have been reported. Moreover, in some cases different calibrations are used for a single observed emission-line flux ratio. For these two reasons care must be taken when comparing relations involving oxygen abundances derived from different methods since the differences could be due to the choice of the calibration rather than to the real abundance.

In this work we study the effect of the aperture on two observational quantities, namely $\mathrm{N} 2\left(\log \left[\mathrm{N}_{\mathrm{II}}\right] \lambda 6583 \AA / \mathrm{H} \alpha\right)$ and O3N2 $\left(\log \left(\left[\mathrm{O}_{\text {III }}\right] \lambda 5007 \AA / \mathrm{H} \beta\right) /\left(\left[\mathrm{N}_{\text {II }}\right] \lambda 6583 \AA / \mathrm{H} \alpha\right)\right)$, that have been widely used in the literature to estimate oxygen abundanes of star formation galaxies (e.g., Pettini \& Pagel 2004; PérezMontero \& Contini 2009; Marino et al. 2013). The applicability intervals of these indicators are $-2.5<\mathrm{N} 2<-0.3$ and $\mathrm{O} 3 \mathrm{~N} 2<2$. Marino et al. (2013) found dispersions of $\sigma \approx 0.16$ and 0.18 dex when fitting temperature-based abundances to abundances derived from the $\mathrm{N} 2$ and $\mathrm{O} 3 \mathrm{~N} 2$ methods using the following calibrations:

$$
12+\log \mathrm{O} / \mathrm{H}=8.743+0.462 \times \mathrm{N} 2
$$

and

$$
12+\log \mathrm{O} / \mathrm{H}=8.533-0.214 \times \mathrm{O} 3 \mathrm{~N} 2 .
$$

Our study is performed for these two observational quantities, so the effect of the aperture on the abundances depends on the choice of the calibration based on any of these observed quantities. A further advantage of this larger sample, compared to the one used in IP13, is that it allows us to study the effect of parameters like inclination, morphological type, and stellar masses, on the derived average aperture corrections.

The paper is organized as follows: Section 2 explains the selection of the sample. The main results from our analysis are detailed in Section 3. Section 4 contains a discussion of the implications of the aperture effects on the abundance determination and an example to illustrate this interesting point. Finally, the conclusions of the paper are enumerated in Section 5. 
Table 4

$x\left(\alpha_{50}\right)$ for Two Bins of Morphological Types Assuming the Same Weight for All Galaxies

\begin{tabular}{|c|c|c|c|c|c|c|c|c|c|}
\hline \multirow{2}{*}{$r / R_{50}$} & \multicolumn{3}{|c|}{$\mathrm{Sa}-\mathrm{Sbc}$} & \multicolumn{3}{|c|}{$\mathrm{Sc}-\mathrm{Sdm}$} & \multicolumn{3}{|c|}{ All } \\
\hline & $-\sigma$ & $x\left(\alpha_{50}\right)$ & $+\sigma$ & $-\sigma$ & $x\left(\alpha_{50}\right)$ & $+\sigma$ & $-\sigma$ & $x\left(\alpha_{50}\right)$ & $+\sigma$ \\
\hline 0.00 & 0.000 & 0.000 & 0.000 & 0.000 & 0.000 & 0.000 & 0.000 & 0.000 & 0.000 \\
\hline 0.10 & 0.007 & 0.021 & 0.066 & 0.009 & 0.018 & 0.032 & 0.008 & 0.020 & 0.057 \\
\hline 0.20 & 0.022 & 0.046 & 0.131 & 0.027 & 0.045 & 0.073 & 0.024 & 0.046 & 0.114 \\
\hline 0.30 & 0.040 & 0.082 & 0.200 & 0.053 & 0.086 & 0.135 & 0.049 & 0.085 & 0.169 \\
\hline 0.40 & 0.072 & 0.130 & 0.262 & 0.084 & 0.132 & 0.213 & 0.077 & 0.130 & 0.246 \\
\hline 0.50 & 0.105 & 0.182 & 0.333 & 0.128 & 0.187 & 0.284 & 0.111 & 0.182 & 0.316 \\
\hline 0.60 & 0.140 & 0.226 & 0.388 & 0.165 & 0.244 & 0.361 & 0.148 & 0.237 & 0.375 \\
\hline 0.70 & 0.176 & 0.282 & 0.456 & 0.212 & 0.297 & 0.435 & 0.193 & 0.283 & 0.445 \\
\hline 0.80 & 0.223 & 0.335 & 0.515 & 0.273 & 0.358 & 0.488 & 0.244 & 0.340 & 0.505 \\
\hline 0.90 & 0.264 & 0.386 & 0.560 & 0.327 & 0.423 & 0.542 & 0.288 & 0.397 & 0.553 \\
\hline 1.00 & 0.312 & 0.441 & 0.610 & 0.371 & 0.484 & 0.608 & 0.329 & 0.454 & 0.609 \\
\hline 1.10 & 0.354 & 0.491 & 0.653 & 0.419 & 0.543 & 0.669 & 0.385 & 0.506 & 0.654 \\
\hline 1.20 & 0.408 & 0.545 & 0.693 & 0.480 & 0.594 & 0.718 & 0.449 & 0.567 & 0.697 \\
\hline 1.30 & 0.457 & 0.606 & 0.731 & 0.544 & 0.638 & 0.770 & 0.504 & 0.620 & 0.746 \\
\hline 1.40 & 0.498 & 0.662 & 0.775 & 0.607 & 0.687 & 0.818 & 0.552 & 0.673 & 0.789 \\
\hline 1.50 & 0.545 & 0.703 & 0.809 & 0.663 & 0.743 & 0.858 & 0.592 & 0.715 & 0.833 \\
\hline 1.60 & 0.583 & 0.737 & 0.838 & 0.698 & 0.788 & 0.884 & 0.645 & 0.759 & 0.862 \\
\hline 1.70 & 0.641 & 0.780 & 0.873 & 0.729 & 0.829 & 0.907 & 0.682 & 0.801 & 0.890 \\
\hline 1.80 & 0.701 & 0.820 & 0.902 & 0.777 & 0.863 & 0.927 & 0.727 & 0.834 & 0.912 \\
\hline 1.90 & 0.747 & 0.853 & 0.923 & 0.815 & 0.898 & 0.937 & 0.773 & 0.865 & 0.930 \\
\hline 2.00 & 0.798 & 0.890 & 0.941 & 0.848 & 0.918 & 0.953 & 0.817 & 0.895 & 0.944 \\
\hline 2.10 & 0.850 & 0.915 & 0.958 & 0.880 & 0.939 & 0.967 & 0.861 & 0.920 & 0.961 \\
\hline 2.20 & 0.891 & 0.940 & 0.971 & 0.913 & 0.957 & 0.982 & 0.899 & 0.943 & 0.975 \\
\hline 2.30 & 0.928 & 0.961 & 0.984 & 0.943 & 0.973 & 0.991 & 0.934 & 0.965 & 0.988 \\
\hline 2.40 & 0.965 & 0.983 & 0.993 & 0.973 & 0.987 & 0.996 & 0.968 & 0.984 & 0.995 \\
\hline 2.50 & 1.000 & 1.000 & 1.000 & 1.000 & 1.000 & 1.000 & 1.000 & 1.000 & 1.000 \\
\hline
\end{tabular}

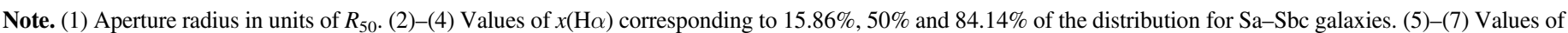

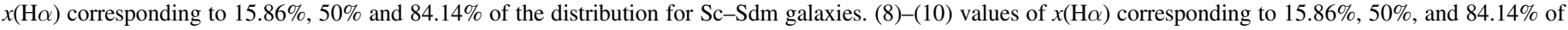
the distribution for all galaxies.

\section{SAMPLE SELECTION}

The sample of galaxies used for this work has been selected from the CALIFA database updated to 2013 December, reduced with the v1.4 version of the pipeline (García-Benito et al. 2015). Details on the instrumental setup and properties of the spectra can be found in Sánchez et al. (2012), where the survey is presented. We started with an initial sample of 402 galaxies, the ones observed up to 2013 December, out of the 937 galaxies comprising the total CALIFA mother sample (Walcher et al. 2014). Then we removed the elliptical and lenticular galaxies (E, S0, S0a) in order to keep only spiral galaxies. As we are interested in covering the galaxy disks as much as possible within the CALIFA aperture $\left(\approx 36^{\prime \prime}\right.$ radius $)$, we have to choose a proper scale related to the spatial extent of the galaxies. From this, two possibilities related to different structural components of the galaxies arise as the most interesting:

1. The Petrosian radius in the SDSS $-r^{\prime}$ band within a circular aperture containing 50\% of the total Petrosian flux (SDSS petroR50_r , and hereafter $R_{50}$ ): this scale takes into account the stellar emission from both the bulge and the disk inside this circular aperture. Thus, this spatial scale is sensitive to light coming from stars of different ages, and has the great advantage of its availability for a huge number of galaxies, in particular the whole CALIFA sample.
2. The effective radius encompasing $50 \%$ of the light coming from the disk component (hereafter $R_{\text {eff }}$ ): this scale is computed after a morphological decomposition bulge/disk of the galaxy SDSS $g^{\prime}$-band surface brightness profile, removing the light coming from the bulge and assuming that the disk has an exponential profile of the form

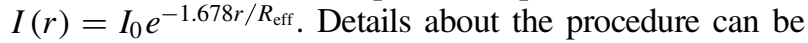
found in Sánchez et al. (2014).

Figure 1 shows the comparison of $R_{50}$ and $R_{\text {eff }}$ with the optical radii, ${ }^{20}$ which correspond to the major semi-axis of the elliptical aperture at $\mu_{B}=25 \mathrm{mag} \operatorname{arcsec}^{-2}$ isophote (hereafter $R_{\mathrm{op}}$ ) of the CALIFA spirals. As the figure shows, for most spiral galaxies $2 R_{50} \leqslant R_{\mathrm{op}} \leqslant 4 R_{50}$, with the exception of the $\mathrm{Sa}-\mathrm{Sab}$ galaxies for most of which $R_{\text {op }}$ seems to be lower than $4 R_{50}$. This differential behavior is likely due to the fact that Sa$\mathrm{Sab}$ galaxies present promiment bulges that result in reduced values of $R_{50}$. In addition, it is shown that $R_{\text {eff }} \leqslant R_{\text {op }} \leqslant 3 R_{\text {eff }}$, and this relation holds in the same way for all morphological types. In this case, all morphological types behave similarly because $R_{\text {eff }}$ is estimated by using only the light from the disk.

In the subsequent analysis we will present the results of the growth curves (as a function of $R_{50}$ and $R_{\text {eff }}$ ) of several observable fluxes or flux ratios based on a selected set of emission lines. They will be used as indicators of the bias induced when estimating galaxy properties from fluxes

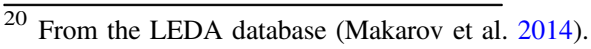



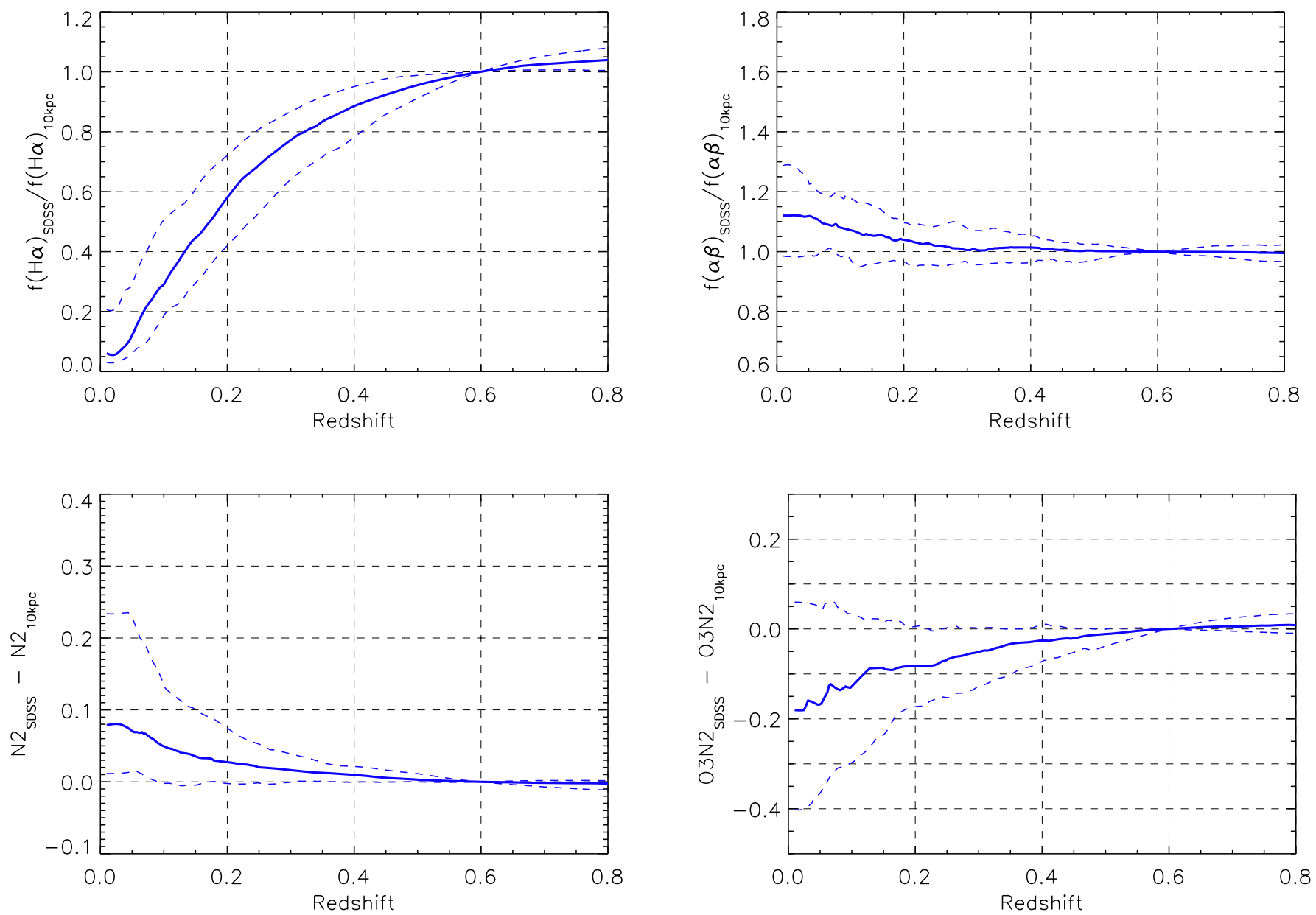

Figure 15. Ratio of the $\mathrm{H} \alpha$ flux (top left), $\mathrm{H} \alpha / \mathrm{H} \beta$ (top right), $\mathrm{N} 2$ (bottom left), and $\mathrm{O} 3 \mathrm{~N} 2$ (bottom right) contained in the SDSS aperture at different redshifts to the corresponding values within a circular aperture of $10 \mathrm{kpc}$ diameter as a function of redshift for the (52) CALIFA spirals whose $10 \mathrm{kpc}$ diameter aperture is completely covered by PMAS/PPAK. Lower dashed, solid, and upper dashed lines correspond to $15.86 \%, 50 \%$, and $84.14 \%$ of the distributions.

measured within reduced apertures instead of taking the integrated values of these fluxes. Hereafter we will refer to the median growth curves of the $\mathrm{H} \alpha$ flux, and the $\mathrm{H} \alpha / \mathrm{H} \beta, \mathrm{N} 2$, and $\mathrm{O} 3 \mathrm{~N} 2$ ratios as a function of $R_{50}$ as $x\left(\alpha_{50}\right), x\left(\alpha \beta_{50}\right)$, $x\left(\mathrm{~N} 2_{50}\right)$, and $x\left(\mathrm{O} 3 \mathrm{~N} 2_{50}\right)$, respectively. These curves represent the value of the parameter measured within a circular aperture of a given radius normalized to the value of the parameter measured within $36^{\prime \prime}$, which is the radius of the largest circular aperture considered. This way, the value of all parameters within a circular aperture of radius $36^{\prime \prime}$ will be taken as the integrated value of this property. In the case of $\mathrm{N} 2$ and $\mathrm{O} 3 \mathrm{~N} 2$, the growth curves are logarithmic. Thus,

$$
\begin{aligned}
x\left(\alpha_{50}\right)_{r}= & f(\mathrm{H} \alpha)_{r} / f(\mathrm{H} \alpha)_{36^{\prime \prime}}, \\
x\left(\alpha \beta_{50}\right)_{r}= & \left(f(\mathrm{H} \alpha)_{r} / f(H \beta)_{r}\right) /\left(f(\mathrm{H} \alpha)_{36^{\prime \prime}} / f(H \beta)_{36^{\prime \prime}}\right), \\
x\left(\mathrm{~N} 2_{50}\right)_{r}= & \log [f([\mathrm{~N} \mathrm{II}] 6583) / f(\mathrm{H} \alpha)]_{r} \\
& -\log [f([\mathrm{~N} \mathrm{II}] 6583) / f(\mathrm{H} \alpha)]_{36^{\prime \prime}},
\end{aligned}
$$

and

$$
\begin{aligned}
x\left(\mathrm{O} 3 \mathrm{~N} 2_{50}\right)_{r} \\
=\log [(f([\mathrm{O} \mathrm{III}] 5007) / f(\mathrm{H} \beta)) /(f([\mathrm{~N} \mathrm{II}] 6583) / f(\mathrm{H} \alpha))]_{r} \\
-\log [(f([\mathrm{O} \mathrm{III}] 5007) / f(\mathrm{H} \beta)) / \\
\quad(f([\mathrm{~N} \mathrm{II}] 6583) / f(\mathrm{H} \alpha))]_{36} .
\end{aligned}
$$

Correspondingly, we will refer to the growth curves of the $\mathrm{H} \alpha$ flux, and the $\mathrm{H} \alpha / \mathrm{H} \beta, \mathrm{N} 2$, and $\mathrm{O} 3 \mathrm{~N} 2$ ratios as a function of $R_{\mathrm{eff}}$ as $x\left(\alpha_{\text {eff }}\right), x\left(\alpha \beta_{\text {eff }}\right), x\left(\mathrm{~N} 2_{\text {eff }}\right)$, and $x\left(\mathrm{O} 3 \mathrm{~N} 2_{\text {eff }}\right)$, respectively.

The procedure followed to produce the growth curves is similar to the one described in IP13. For each galaxy, unidimensional spectra are constructed by adding all the pixels within circular apertures of radii varying from $3^{\prime \prime}$ to $36^{\prime \prime}$, in steps of $3^{\prime \prime}$. In order to obtain the pure emission-line spectra, we use a single stellar population (SSP) fitting to remove the contribution of the underlying continuum of the stellar population. We apply a linear combination of two SSP synthesis models of Vazdekis et al. (2010) based on the MILES stellar library (Sánchez-Blázquez et al. 2006) and a Kroupa IMF (Kroupa 2001). The ages of the SSPs used range from 0.10 to $0.79 \mathrm{Gyr}$ for the case of the young stellar population and from 2.00 to $14.13 \mathrm{Gyr}$ for the old stellar population. Five different metallicities are considered for each age $([\mathrm{M} / \mathrm{H}]$ value equal to $0.00,0.20,-0.40,-0.71$, and $-1.31 \mathrm{dex}$ offset from the solar value). Once the underlying stellar continuum is removed, the fluxes of the emission lines were obtained from Gaussian fits to the residual spectra. The procedure followed to get the fits is as follows: first we fit the triplet $\mathrm{H} \alpha+[\mathrm{N}$ II] assuming a common recession velocity and FWHM for the three lines, and the usual line ratio $f(6548) / f(6583)=0.333$. No broad components in $\mathrm{H} \alpha$ were considered because we have removed 

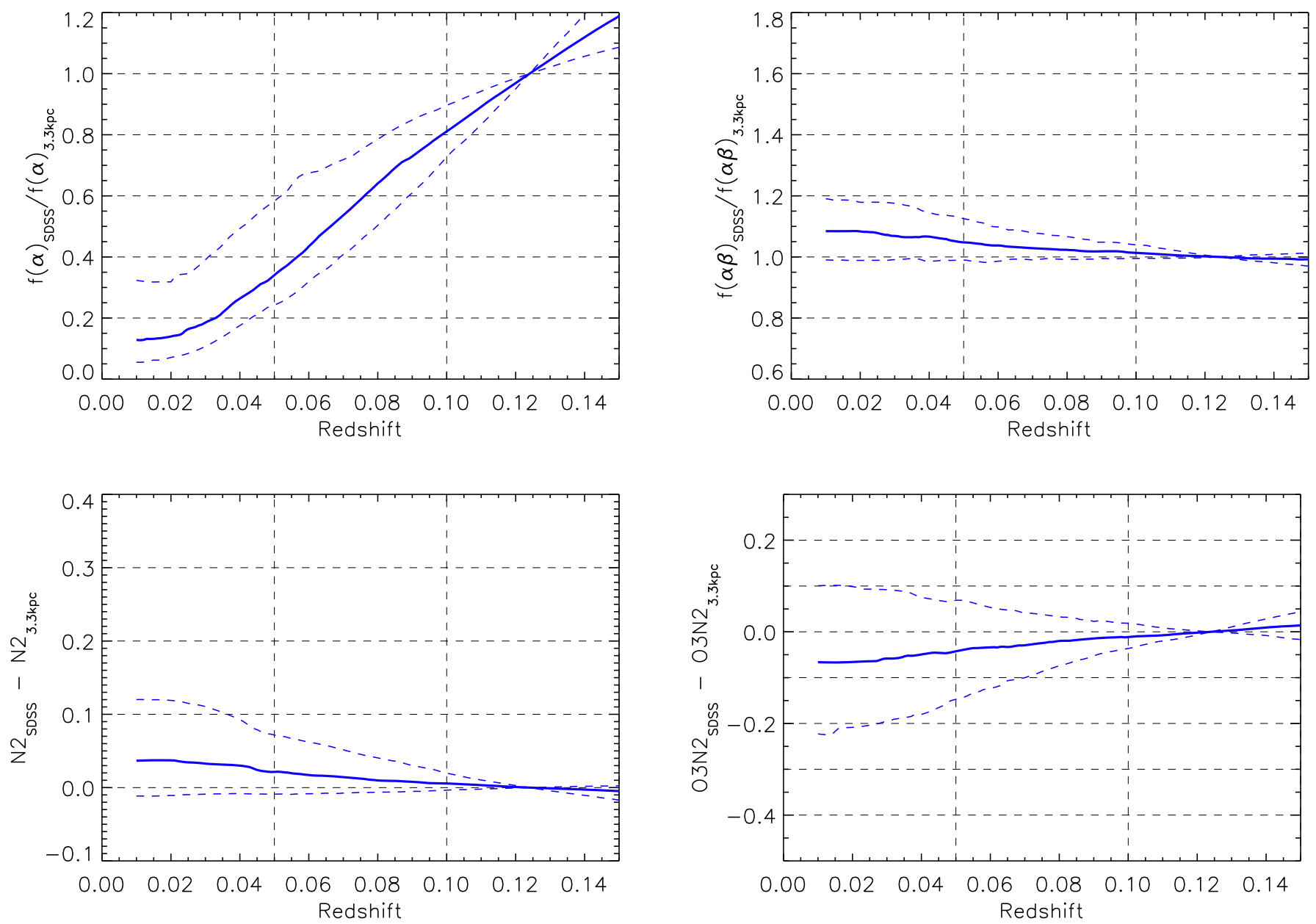

Figure 16. Ratio of the $\mathrm{H} \alpha$ flux (top left), $\mathrm{H} \alpha / \mathrm{H} \beta$ (top right), $\mathrm{N} 2$ (bottom left), and $\mathrm{O} 3 \mathrm{~N} 2$ (bottom right) contained in the SDSS aperture at different redshifts to the corresponding values within a circular aperture of $3.3 \mathrm{kpc}$ diameter as a function of redshift for the (96) CALIFA spirals whose $3.3 \mathrm{kpc}$ diameter aperture is completely covered by PMAS/PPAK. Lower dashed, solid, and upper dashed lines correspond to $15.86 \%, 50 \%$, and $84.14 \%$ of the distributions.

Table 5

$x\left(\alpha_{\text {eff }}\right)$ for Two Bins of Morphological Types Assuming the Same Weight for All Galaxies

\begin{tabular}{|c|c|c|c|c|c|c|c|c|c|}
\hline \multirow{2}{*}{$r / R_{\text {eff }}$} & \multicolumn{3}{|c|}{$\mathrm{Sa}-\mathrm{Sbc}$} & \multicolumn{3}{|c|}{ Sc-Sdm } & \multicolumn{3}{|c|}{ All } \\
\hline & $-\sigma$ & $x\left(\alpha_{\mathrm{eff}}\right)$ & $+\sigma$ & $-\sigma$ & $x\left(\alpha_{\mathrm{eff}}\right)$ & $+\sigma-\sigma$ & $x\left(\alpha_{\text {eff }}\right)$ & $+\sigma$ & \\
\hline 0.00 & 0.000 & 0.000 & 0.000 & 0.000 & 0.000 & 0.000 & 0.000 & 0.000 & 0.000 \\
\hline 0.10 & 0.016 & 0.038 & 0.142 & 0.018 & 0.040 & 0.080 & 0.017 & 0.039 & 0.107 \\
\hline 0.20 & 0.049 & 0.109 & 0.237 & 0.057 & 0.100 & 0.189 & 0.054 & 0.107 & 0.227 \\
\hline 0.30 & 0.103 & 0.210 & 0.364 & 0.113 & 0.184 & 0.310 & 0.103 & 0.195 & 0.335 \\
\hline 0.40 & 0.173 & 0.284 & 0.471 & 0.189 & 0.271 & 0.425 & 0.177 & 0.280 & 0.457 \\
\hline 0.50 & 0.254 & 0.388 & 0.589 & 0.264 & 0.365 & 0.531 & 0.256 & 0.385 & 0.561 \\
\hline 0.60 & 0.337 & 0.478 & 0.670 & 0.333 & 0.455 & 0.623 & 0.336 & 0.468 & 0.664 \\
\hline 0.70 & 0.427 & 0.577 & 0.724 & 0.403 & 0.535 & 0.728 & 0.417 & 0.568 & 0.727 \\
\hline 0.80 & 0.536 & 0.658 & 0.781 & 0.505 & 0.634 & 0.804 & 0.535 & 0.655 & 0.782 \\
\hline 0.90 & 0.613 & 0.715 & 0.834 & 0.583 & 0.735 & 0.869 & 0.612 & 0.722 & 0.835 \\
\hline 1.00 & 0.700 & 0.785 & 0.880 & 0.669 & 0.799 & 0.896 & 0.684 & 0.794 & 0.889 \\
\hline 1.10 & 0.775 & 0.853 & 0.922 & 0.750 & 0.848 & 0.934 & 0.769 & 0.851 & 0.925 \\
\hline 1.20 & 0.845 & 0.905 & 0.945 & 0.828 & 0.897 & 0.967 & 0.842 & 0.902 & 0.958 \\
\hline 1.30 & 0.901 & 0.940 & 0.976 & 0.905 & 0.939 & 0.984 & 0.903 & 0.940 & 0.981 \\
\hline 1.40 & 0.956 & 0.972 & 0.991 & 0.956 & 0.977 & 0.994 & 0.956 & 0.974 & 0.992 \\
\hline 1.50 & 1.000 & 1.000 & 1.000 & 1.000 & 1.000 & 1.000 & 1.000 & 1.000 & 1.000 \\
\hline
\end{tabular}

Note. (1) Aperture radius in units of $R_{\text {eff }}$. (2)-(4) Values of $x(\mathrm{H} \alpha)$ corresponding to $15.86 \%, 50 \%$, and $84.14 \%$ of the distribution for Sa-Sbc galaxies. (5)-(7) Values of $x(\mathrm{H} \alpha)$ corresponding to $15.86 \%, 50 \%$ and $84.14 \%$ of the distribution for Sc-Sdm galaxies. (8)-(10) values of $x(\mathrm{H} \alpha)$ corresponding to $15.86 \%, 50 \%$, and $84.14 \%$ of the distribution for all galaxies. 
Table 6

$x\left(\alpha_{50}\right)$ for Two Bins of Stellar Masses Assuming the Same Weight for All Galaxies

\begin{tabular}{|c|c|c|c|c|c|c|}
\hline \multirow{2}{*}{$r / R_{50}$} & \multicolumn{3}{|c|}{$\log M^{*} \leqslant 10.3$} & \multicolumn{3}{|c|}{$\log M^{*}>10.3$} \\
\hline & $-\sigma$ & $x\left(\alpha_{50}\right)$ & $+\sigma$ & $-\sigma$ & $x\left(\alpha_{50}\right)$ & $+\sigma$ \\
\hline 0.00 & 0.000 & 0.000 & 0.000 & 0.000 & 0.000 & 0.000 \\
\hline 0.10 & 0.009 & 0.020 & 0.039 & 0.007 & 0.020 & 0.066 \\
\hline 0.20 & 0.027 & 0.046 & 0.083 & 0.021 & 0.048 & 0.132 \\
\hline 0.30 & 0.052 & 0.085 & 0.146 & 0.039 & 0.084 & 0.202 \\
\hline 0.40 & 0.083 & 0.133 & 0.231 & 0.070 & 0.130 & 0.263 \\
\hline 0.50 & 0.120 & 0.186 & 0.298 & 0.104 & 0.182 & 0.331 \\
\hline 0.60 & 0.164 & 0.237 & 0.372 & 0.138 & 0.231 & 0.379 \\
\hline 0.70 & 0.211 & 0.286 & 0.455 & 0.175 & 0.281 & 0.421 \\
\hline 0.80 & 0.260 & 0.352 & 0.516 & 0.218 & 0.338 & 0.483 \\
\hline 0.90 & 0.309 & 0.412 & 0.564 & 0.254 & 0.389 & 0.547 \\
\hline 1.00 & 0.363 & 0.463 & 0.629 & 0.309 & 0.443 & 0.593 \\
\hline 1.10 & 0.420 & 0.515 & 0.694 & 0.347 & 0.496 & 0.649 \\
\hline 1.20 & 0.478 & 0.579 & 0.731 & 0.401 & 0.562 & 0.687 \\
\hline 1.30 & 0.532 & 0.624 & 0.766 & 0.444 & 0.617 & 0.729 \\
\hline 1.40 & 0.587 & 0.679 & 0.818 & 0.491 & 0.668 & 0.764 \\
\hline 1.50 & 0.630 & 0.725 & 0.856 & 0.545 & 0.707 & 0.804 \\
\hline 1.60 & 0.658 & 0.769 & 0.883 & 0.583 & 0.752 & 0.837 \\
\hline 1.70 & 0.710 & 0.822 & 0.906 & 0.634 & 0.794 & 0.872 \\
\hline 1.80 & 0.743 & 0.852 & 0.927 & 0.695 & 0.829 & 0.897 \\
\hline 1.90 & 0.796 & 0.884 & 0.941 & 0.746 & 0.862 & 0.920 \\
\hline 2.00 & 0.834 & 0.907 & 0.955 & 0.797 & 0.891 & 0.938 \\
\hline 2.10 & 0.867 & 0.928 & 0.970 & 0.849 & 0.919 & 0.956 \\
\hline 2.20 & 0.903 & 0.947 & 0.981 & 0.891 & 0.943 & 0.971 \\
\hline 2.30 & 0.939 & 0.965 & 0.989 & 0.929 & 0.965 & 0.984 \\
\hline 2.40 & 0.970 & 0.984 & 0.995 & 0.964 & 0.984 & 0.994 \\
\hline 2.50 & 1.000 & 1.000 & 1.000 & 1.000 & 1.000 & 1.000 \\
\hline
\end{tabular}

Note. (1) Aperture radius in units of $R_{50}$. (2)-(4) Values of $x(\mathrm{H} \alpha)$ corresponding to $15.86 \%, 50 \%$, and $84.14 \%$ of the distribution for $\log M^{*} \leqslant 10.3$ galaxies. (5)(7) Values of $x(\mathrm{H} \alpha)$ corresponding to $15.86 \%, 50 \%$, and $84.14 \%$ of the distribution for $\log M^{*}>10.3$ galaxies.

Table 7

$x\left(\alpha_{\text {eff }}\right)$ for Two Bins of Stellar Masses Assuming the Same Weight for All Galaxies

\begin{tabular}{lccccccc}
\hline \hline \multirow{2}{*}{$r / R_{\text {eff }}$} & \multicolumn{3}{c}{$\log M^{*} \leqslant 10.3$} & & \multicolumn{3}{c}{$\log M^{*}>10.3$} \\
\cline { 2 - 3 } \cline { 6 - 7 } & $-\sigma$ & $x\left(\alpha_{\text {eff }}\right)$ & $+\sigma$ & & $-\sigma$ & $x\left(\alpha_{\text {eff }}\right)$ & $+\sigma$ \\
\hline 0.00 & 0.000 & 0.000 & 0.000 & & 0.000 & 0.000 & 0.000 \\
0.10 & 0.019 & 0.040 & 0.091 & & 0.016 & 0.035 & 0.114 \\
0.20 & 0.057 & 0.110 & 0.229 & & 0.046 & 0.096 & 0.226 \\
0.30 & 0.116 & 0.203 & 0.375 & & 0.103 & 0.180 & 0.332 \\
0.40 & 0.191 & 0.293 & 0.464 & & 0.165 & 0.263 & 0.452 \\
0.50 & 0.273 & 0.404 & 0.587 & & 0.235 & 0.366 & 0.557 \\
0.60 & 0.357 & 0.498 & 0.667 & 0.322 & 0.465 & 0.638 \\
0.70 & 0.441 & 0.590 & 0.732 & & 0.415 & 0.561 & 0.718 \\
0.80 & 0.525 & 0.657 & 0.815 & 0.536 & 0.643 & 0.775 \\
0.90 & 0.612 & 0.744 & 0.871 & 0.612 & 0.713 & 0.831 \\
1.00 & 0.683 & 0.803 & 0.905 & 0.685 & 0.784 & 0.867 \\
1.10 & 0.768 & 0.851 & 0.933 & 0.771 & 0.850 & 0.922 \\
1.20 & 0.841 & 0.906 & 0.966 & 0.843 & 0.898 & 0.938 \\
1.30 & 0.903 & 0.946 & 0.982 & 0.902 & 0.938 & 0.970 \\
1.40 & 0.956 & 0.977 & 0.994 & 0.957 & 0.973 & 0.990 \\
1.50 & 1.000 & 1.000 & 1.000 & 1.000 & 1.000 & 1.000 \\
\hline
\end{tabular}

Note. (1) Aperture radius in units of $R_{\text {eff }}$. (2)-(4) Values of $x(\mathrm{H} \alpha)$ corresponding to $15.86 \%, 50 \%$, and $84.14 \%$ of the distribution for $\log M^{*} \leqslant 10.3$ galaxies. (5)(7) Values of $x(\mathrm{H} \alpha)$ corresponding to $15.86 \%, 50 \%$, and $84.14 \%$ of the distribution for $\log M^{*}>10.3$ galaxies. active galactic nuclei (AGNs) from our samples and thus only narrow emission lines are expected. Then we produced individual fits for $\mathrm{H} \beta$ and $[\mathrm{O}$ III] leaving free the recession velocity and the FWHM but using as a first guess the values obtained for $\mathrm{H} \alpha$ $+[\mathrm{N}$ II $]$. After that, for each galaxy we have 12 element vectors containing the fluxes of the emission lines contained within each of the circular apertures. The median growth curves as a function of $R_{50}$ or $R_{\text {eff }}$ where then produced by combining the properly interpolated vectors.

In addition to this, given that we focus on star-forming galaxies, for the subsequent analysis we remove those CALIFA galaxies classified as AGNs, according to the prescriptions of Best \& Heckman (2012), Kewley et al. (2001, 2006), and Cid Fernandes et al. (2011), and to the $\mathrm{NED}^{21}$ database. In order to keep only galaxies with good-quality data we will remove those galaxies for which $f(\mathrm{H} \alpha)>0$ and $\sigma(\mathrm{H} \alpha) / f(\mathrm{H} \alpha) \leqslant 0.333$ in all the circular apertures. We note that the results of this study are the same if we apply a 2- $\sigma$ cut in signal-to-noise ratio, resulting in a significantly larger sample, instead of the 3- $\sigma$ applied in what follows.

As a first step we investigate the distribution of the $\mathrm{H} \alpha$ emission in the CALIFA galaxies. Figure 2 shows $x\left(\alpha_{50}\right)$ and $x\left(\alpha_{\text {eff }}\right)$ for the spirals covered in the CALIFA aperture up to $4 R_{50}$ and $2.2 R_{\text {eff }}$, respectively. These limits were imposed with the compromise of covering as much as possible the disks of the CALIFA galaxies, but keeping samples with reasonable numbers of galaxies. In both cases, $x\left(\alpha_{50}\right)$ and $x\left(\alpha_{\text {eff }}\right)$ seem to saturate at $4 R_{50}$ and $2.2 R_{\text {eff }}$, respectively, suggesting that the bulk of the $\mathrm{H} \alpha$ emission of spiral galaxies is contained within these apertures. But the numbers of galaxies available for this test are very low for a detailed study of the aperture effects including the role of the stellar mass and morphological type. For this reason, we chose two less conservative limits, namely $2.5 R_{50}$ and $1.5 R_{\text {eff }}$, that contain on average $85 \%$ and $90 \%$ of the total $\mathrm{H} \alpha$ emission in spiral galaxies (see Figure 2).

After imposing this last condition, we end up with 165 galaxies covered up to $2.5 R_{50}$ and 133 galaxies covered up to $1.5 R_{\text {eff }}$, which is the case for galaxies with $R_{50} \leqslant 14$ !" 4 and $R_{\text {eff }} \leqslant 24^{\prime \prime}$, respectively. $^{23}$ These two subsamples $\left(S_{50}\right.$ and $S_{\text {eff }}$ ) will be the basis of our statistical study of aperture effects, taking into account that $S_{50}$ galaxies will be studied up to $\approx 2.5 R_{50}$, which contains on average $85 \%$ of their $\mathrm{H} \alpha$ emission, and that $S_{\text {eff }}$ galaxies will be be studied up to $\approx 1.5 R_{\text {eff }}$, which contains on average $90 \%$ of their $\mathrm{H} \alpha$ emission. Table 1 shows the number of galaxies of each subsample after imposing each of the filters previously mentioned. Also, Table 2 contains some basic properties of the two subsamples.

\footnotetext{
21 http://ned.ipac.caltech.edu/

3 This value is slightly lower than the one, $90 \%$, reported in IP13. Given that the current sample is larger, we are more confident about the value reported in this paper.

${ }^{23}$ Further restrictions must be imposed to the subsamples when analyzing the $\mathrm{H} \alpha / \mathrm{H} \beta, \mathrm{N} 2$ and $\mathrm{O} 3 \mathrm{~N} 2$ growth curves, namely $f(\mathrm{H} \beta)>0, \sigma(\mathrm{H} \beta) / f(\mathrm{H} \beta) \leqslant$ $0.333, f([\mathrm{~N}$ II $])>0, \sigma([\mathrm{N} \mathrm{II}]) / f([\mathrm{~N} \mathrm{III}]) \leqslant 0.333, f([\mathrm{O}$ III] $])>0$, and $\sigma([\mathrm{O}$ III] $]) / f$ $([\mathrm{O} \mathrm{III}]) \leqslant 0.333$, in all circular apertures. These restrictions shorten $S_{50}$ to 159 106 and 104 galaxies, and $S_{\text {eff }}$ to 129,92 and 90 galaxies respectively.
} 
Table 8

$x\left(\alpha \beta_{50}\right)$ for Two Bins of Morphological Types Assuming the Same Weight for All Galaxies

\begin{tabular}{|c|c|c|c|c|c|c|c|c|c|}
\hline \multirow{2}{*}{$r / R_{50}$} & \multicolumn{3}{|c|}{$\mathrm{Sa}-\mathrm{Sbc}$} & \multicolumn{3}{|c|}{ Sc-Sdm } & \multicolumn{3}{|c|}{ All } \\
\hline & $-\sigma$ & $x\left(\alpha \beta_{50}\right)$ & $+\sigma$ & $-\sigma$ & $x\left(\alpha \beta_{50}\right)$ & $+\sigma$ & $-\sigma$ & $x\left(\alpha \beta_{50}\right)$ & $+\sigma$ \\
\hline 0.00 & 1.006 & 1.144 & 1.391 & 0.965 & 1.087 & 1.234 & 0.992 & 1.123 & 1.333 \\
\hline 0.10 & 0.996 & 1.153 & 1.404 & 0.962 & 1.092 & 1.244 & 0.985 & 1.123 & 1.351 \\
\hline 0.20 & 0.998 & 1.149 & 1.399 & 0.964 & 1.094 & 1.241 & 0.984 & 1.126 & 1.352 \\
\hline 0.30 & 1.010 & 1.153 & 1.369 & 0.987 & 1.092 & 1.233 & 0.993 & 1.118 & 1.328 \\
\hline 0.40 & 1.007 & 1.132 & 1.304 & 0.995 & 1.079 & 1.220 & 0.996 & 1.104 & 1.284 \\
\hline 0.50 & 0.992 & 1.113 & 1.260 & 0.994 & 1.059 & 1.200 & 0.993 & 1.083 & 1.234 \\
\hline 0.80 & 0.952 & 1.086 & 1.191 & 0.993 & 1.036 & 1.147 & 0.972 & 1.061 & 1.175 \\
\hline 0.90 & 0.944 & 1.069 & 1.166 & 0.980 & 1.035 & 1.117 & 0.972 & 1.055 & 1.162 \\
\hline 1.00 & 0.956 & 1.059 & 1.147 & 0.970 & 1.036 & 1.104 & 0.960 & 1.041 & 1.143 \\
\hline 1.10 & 0.966 & 1.049 & 1.138 & 0.972 & 1.025 & 1.099 & 0.968 & 1.034 & 1.131 \\
\hline 1.20 & 0.967 & 1.037 & 1.126 & 0.975 & 1.016 & 1.096 & 0.972 & 1.027 & 1.120 \\
\hline 1.30 & 0.969 & 1.034 & 1.128 & 0.966 & 1.014 & 1.079 & 0.968 & 1.023 & 1.109 \\
\hline 1.80 & 0.960 & 1.016 & 1.078 & 0.953 & 0.999 & 1.043 & 0.958 & 1.011 & 1.059 \\
\hline 1.90 & 0.972 & 1.014 & 1.070 & 0.951 & 0.999 & 1.046 & 0.964 & 1.007 & 1.064 \\
\hline 2.00 & 0.977 & 1.015 & 1.058 & 0.958 & 0.998 & 1.051 & 0.972 & 1.010 & 1.052 \\
\hline 2.10 & 0.982 & 1.014 & 1.046 & 0.966 & 1.001 & 1.038 & 0.972 & 1.010 & 1.046 \\
\hline 2.20 & 0.982 & 1.009 & 1.037 & 0.969 & 1.001 & 1.034 & 0.975 & 1.007 & 1.037 \\
\hline 2.30 & 0.986 & 1.007 & 1.029 & 0.970 & 1.000 & 1.024 & 0.980 & 1.005 & 1.025 \\
\hline 2.40 & 0.992 & 1.002 & 1.016 & 0.986 & 0.999 & 1.015 & 0.990 & 1.001 & 1.016 \\
\hline 2.50 & 1.000 & 1.000 & 1.000 & 1.000 & 1.000 & 1.000 & 1.000 & 1.000 & 1.000 \\
\hline
\end{tabular}

Note. (1) Aperture radius in units of $R_{50}$. (2)-(4) Values of $x\left(\alpha \beta_{50}\right)$ corresponding to $15.86 \%, 50 \%$, and $84.14 \%$ of the distribution for Sa-Sbc galaxies. (5)-(7) values of $x\left(\alpha \beta_{50}\right)$ corresponding to $15.86 \%, 50 \%$, and $84.14 \%$ of the distribution for Sc-Sdm galaxies. (8)-(10) Values of $x\left(\alpha \beta_{50}\right)$ corresponding to $15.86 \%$, 50\%, and $84.14 \%$ of the distribution for all galaxies.

Table 9

$x\left(\alpha \beta_{\text {eff }}\right)$ for Two Bins of Morphological Types Assuming the Same Weight for All Galaxies

\begin{tabular}{|c|c|c|c|c|c|c|c|c|c|}
\hline \multirow{2}{*}{$r / R_{\text {eff }}$} & \multicolumn{3}{|c|}{$\mathrm{Sa}-\mathrm{Sbc}$} & \multicolumn{3}{|c|}{ Sc-Sdm } & \multicolumn{3}{|c|}{ All } \\
\hline & $-\sigma$ & $x\left(\alpha \beta_{\text {eff }}\right)$ & $+\sigma$ & $-\sigma$ & $x\left(\alpha \beta_{\text {eff }}\right)$ & $+\sigma$ & $-\sigma$ & $x\left(\alpha \beta_{\text {eff }}\right)$ & $+\sigma$ \\
\hline 0.00 & 0.968 & 1.114 & 1.369 & 0.940 & 1.101 & 1.196 & 0.965 & 1.109 & 1.320 \\
\hline 0.10 & 0.974 & 1.116 & 1.387 & 0.933 & 1.102 & 1.192 & 0.958 & 1.110 & 1.330 \\
\hline 0.20 & 0.966 & 1.110 & 1.311 & 0.941 & 1.093 & 1.208 & 0.954 & 1.104 & 1.295 \\
\hline 0.30 & 0.968 & 1.085 & 1.216 & 0.974 & 1.067 & 1.210 & 0.969 & 1.076 & 1.213 \\
\hline 0.40 & 0.963 & 1.080 & 1.201 & 0.965 & 1.047 & 1.123 & 0.965 & 1.064 & 1.180 \\
\hline 0.50 & 0.981 & 1.064 & 1.173 & 0.970 & 1.044 & 1.107 & 0.975 & 1.055 & 1.140 \\
\hline 0.60 & 0.969 & 1.058 & 1.149 & 0.961 & 1.034 & 1.092 & 0.965 & 1.041 & 1.137 \\
\hline 0.70 & 0.960 & 1.044 & 1.134 & 0.953 & 1.022 & 1.091 & 0.957 & 1.032 & 1.129 \\
\hline 0.80 & 0.965 & 1.041 & 1.112 & 0.947 & 1.014 & 1.101 & 0.959 & 1.021 & 1.109 \\
\hline 0.90 & 0.957 & 1.032 & 1.116 & 0.952 & 1.015 & 1.060 & 0.953 & 1.019 & 1.110 \\
\hline 1.00 & 0.963 & 1.026 & 1.092 & 0.970 & 1.007 & 1.067 & 0.965 & 1.016 & 1.085 \\
\hline 1.10 & 0.955 & 1.018 & 1.086 & 0.954 & 1.009 & 1.055 & 0.955 & 1.012 & 1.076 \\
\hline 1.20 & 0.956 & 1.018 & 1.069 & 0.961 & 1.004 & 1.058 & 0.960 & 1.009 & 1.068 \\
\hline 1.30 & 0.964 & 1.008 & 1.058 & 0.967 & 1.000 & 1.042 & 0.964 & 1.005 & 1.053 \\
\hline 1.40 & 0.980 & 1.005 & 1.033 & 0.983 & 1.001 & 1.021 & 0.982 & 1.002 & 1.025 \\
\hline 1.50 & 1.000 & 1.000 & 1.000 & 1.000 & 1.000 & 1.000 & 1.000 & 1.000 & 1.000 \\
\hline
\end{tabular}

Note. (1) Aperture radius in units of $R_{\text {eff }}$. (2)-(4) Values of $x\left(\alpha \beta_{\text {eff }}\right)$ corresponding to $15.86 \%, 50 \%$, and $84.14 \%$ of the distribution for Sa-Sbc galaxies. (5)-(7) Values of $x\left(\alpha \beta_{\text {eff }}\right)$ corresponding to $15.86 \%, 50 \%$, and $84.14 \%$ of the distribution for Sc-Sdm galaxies. (8)-(10) Values of $x\left(\alpha \beta_{\text {eff }}\right)$ corresponding to $15.86 \%$, 50\%, and $84.14 \%$ of the distribution for all galaxies. 
Table 10

$x\left(\alpha \beta_{50}\right)$ for Two Bins of Stellar Masses Assuming the Same Weight for All Galaxies

\begin{tabular}{|c|c|c|c|c|c|c|}
\hline \multirow{2}{*}{$r / R_{50}$} & \multicolumn{3}{|c|}{$\log M^{*} \leqslant 10.3$} & \multicolumn{3}{|c|}{$\log M^{*}>10.3$} \\
\hline & $-\sigma$ & $x\left(\alpha \beta_{50}\right)$ & $+\sigma$ & $-\sigma$ & $x\left(\alpha \beta_{50}\right)$ & $+\sigma$ \\
\hline 0.00 & 0.989 & 1.102 & 1.257 & 1.005 & 1.178 & $\overline{1.441}$ \\
\hline 0.10 & 0.981 & 1.100 & 1.267 & 0.998 & 1.181 & 1.461 \\
\hline 0.20 & 0.982 & 1.101 & 1.263 & 1.000 & 1.181 & 1.461 \\
\hline 0.30 & 0.993 & 1.101 & 1.255 & 1.003 & 1.167 & 1.430 \\
\hline 0.40 & 0.996 & 1.081 & 1.216 & 1.005 & 1.149 & 1.340 \\
\hline 0.50 & 0.995 & 1.063 & 1.199 & 0.992 & 1.124 & 1.287 \\
\hline 0.60 & 0.999 & 1.046 & 1.197 & 0.969 & 1.117 & 1.257 \\
\hline 0.70 & 0.985 & 1.047 & 1.175 & 0.972 & 1.104 & 1.233 \\
\hline 0.80 & 0.980 & 1.040 & 1.158 & 0.953 & 1.098 & 1.229 \\
\hline 0.90 & 0.977 & 1.040 & 1.134 & 0.954 & 1.075 & 1.214 \\
\hline 1.00 & 0.969 & 1.037 & 1.129 & 0.958 & 1.062 & 1.182 \\
\hline 1.10 & 0.968 & 1.029 & 1.111 & 0.969 & 1.057 & 1.160 \\
\hline 1.20 & 0.963 & 1.021 & 1.105 & 0.981 & 1.041 & 1.150 \\
\hline 1.30 & 0.961 & 1.014 & 1.090 & 0.977 & 1.041 & 1.138 \\
\hline 1.40 & 0.957 & 1.014 & 1.082 & 0.967 & 1.038 & 1.126 \\
\hline 1.50 & 0.961 & 1.011 & 1.079 & 0.953 & 1.036 & 1.109 \\
\hline 1.60 & 0.960 & 1.011 & 1.054 & 0.961 & 1.032 & 1.092 \\
\hline 1.70 & 0.958 & 1.003 & 1.048 & 0.965 & 1.025 & 1.091 \\
\hline 1.80 & 0.948 & 1.002 & 1.045 & 0.965 & 1.022 & 1.079 \\
\hline 1.90 & 0.952 & 1.000 & 1.044 & 0.976 & 1.020 & 1.088 \\
\hline 2.00 & 0.958 & 1.000 & 1.044 & 0.979 & 1.022 & 1.068 \\
\hline 2.10 & 0.966 & 1.002 & 1.037 & 0.982 & 1.017 & 1.049 \\
\hline 2.20 & 0.969 & 1.002 & 1.036 & 0.981 & 1.011 & 1.040 \\
\hline 2.30 & 0.971 & 1.001 & 1.024 & 0.984 & 1.008 & 1.030 \\
\hline 2.40 & 0.987 & 1.000 & 1.014 & 0.993 & 1.002 & 1.016 \\
\hline 2.50 & 1.000 & 1.000 & 1.000 & 1.000 & 1.000 & 1.000 \\
\hline
\end{tabular}

Note. (1) Aperture radius in units of $R_{50}$. (2)-(4) Values of $x\left(\alpha \beta_{50}\right)$ corresponding to $15.86 \%, 50 \%$, and $84.14 \%$ of the distribution for $\log M^{*} \leqslant 10.3$ galaxies. (5)-(7) Values of $x\left(\alpha \beta_{50}\right)$ corresponding to $15.86 \%, 50 \%$, and $84.14 \%$ of the distribution for $\log M^{*}>10.3$ galaxies.

Table 11

$x\left(\alpha \beta_{\text {eff }}\right)$ for Two Bins of Stellar Masses Assuming the Same Weight for All Galaxies

\begin{tabular}{lccccccc}
\hline \hline \multirow{2}{*}{$r / R_{\text {eff }}$} & \multicolumn{3}{c}{$\log M^{*} \leqslant 10.3$} & & \multicolumn{3}{c}{$\log M^{*}>10.3$} \\
\cline { 2 - 4 } \cline { 6 - 7 } & $-\sigma$ & $x\left(\alpha \beta_{\text {eff }}\right)$ & $+\sigma$ & & $-\sigma$ & $x\left(\alpha \beta_{\text {eff }}\right)$ & $+\sigma$ \\
\hline 0.00 & 0.941 & 1.094 & 1.226 & & 0.969 & 1.145 & 1.370 \\
0.10 & 0.941 & 1.099 & 1.239 & & 0.975 & 1.146 & 1.393 \\
0.20 & 0.941 & 1.084 & 1.219 & & 0.984 & 1.126 & 1.320 \\
0.30 & 0.964 & 1.057 & 1.203 & & 0.995 & 1.096 & 1.232 \\
0.40 & 0.956 & 1.047 & 1.144 & & 0.979 & 1.090 & 1.210 \\
0.50 & 0.973 & 1.044 & 1.115 & & 0.981 & 1.073 & 1.179 \\
0.60 & 0.963 & 1.036 & 1.093 & & 0.968 & 1.064 & 1.166 \\
0.70 & 0.956 & 1.030 & 1.093 & & 0.959 & 1.039 & 1.145 \\
0.80 & 0.958 & 1.020 & 1.102 & & 0.959 & 1.023 & 1.122 \\
0.90 & 0.962 & 1.018 & 1.102 & & 0.952 & 1.023 & 1.117 \\
1.00 & 0.971 & 1.016 & 1.080 & & 0.962 & 1.020 & 1.097 \\
1.10 & 0.967 & 1.010 & 1.071 & & 0.952 & 1.018 & 1.093 \\
1.20 & 0.959 & 1.009 & 1.065 & & 0.960 & 1.009 & 1.069 \\
1.30 & 0.972 & 1.004 & 1.051 & & 0.958 & 1.006 & 1.054 \\
1.40 & 0.986 & 1.003 & 1.025 & & 0.977 & 1.002 & 1.028 \\
1.50 & 1.000 & 1.000 & 1.000 & & 1.000 & 1.000 & 1.000 \\
\hline
\end{tabular}

Note. (1) Aperture radius in units of $R_{\text {eff }}$. (2)-(4) Values of $x\left(\alpha \beta_{\text {eff }}\right)$ corresponding to $15.86 \%, 50 \%$, and $84.14 \%$ of the distribution for $\log M^{*} \leqslant$ 10.3 galaxies. (5)-(7) Values of $x\left(\alpha \beta_{\text {eff }}\right)$ corresponding to $15.86 \%, 50 \%$, and $84.14 \%$ of the distribution for $\log M^{*}>10.3$ galaxies.

\section{RESULTS}

\subsection{Corrections for Fixed Angular Apertures}

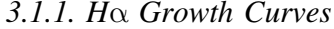

We start discussing the behavior of the growth curves of $x\left(\alpha_{50}\right)$ and $x\left(\alpha_{\text {eff }}\right)$ of our galaxies. For this we first compare $x\left(\alpha_{50}\right)$ of IP13 with that obtained in this work. As in IP13, for each radius we produce median growth curves and a confidence interval equivalent to $1 \sigma$, which contains $68 \%$ of the individual growth curves. Table 3 lists the values of $x\left(\alpha_{50}\right)$ of IP13 and the one produced with the present sample. As can be seen from Table 3, both curves are consistent within $1 \sigma$ along the probed range. The increased number of galaxies in our current sample will make possible a more detailed analysis of the aperture corrections split by morphological types or stellar masses.

Figures 3-5 show the $\mathrm{H} \alpha$ growth curves split by galaxy inclination, morphological type, and stellar mass. Inclination is defined as the ratio of SDSS isob_r/isoa_r and we consider face-on galaxies to be those with inclination larger than 0.4, the rest being edge-on galaxies. Concerning the morphological types, two bins were defined as follows: $\mathrm{Sa}$ to $\mathrm{Sbc}$ and $\mathrm{Sc}$ to Sdm. This splits our sample into early spirals and late spirals. Finally, the stellar mass was also split into two ranges, separated at $\log M^{*} / M_{\odot}=10.3$. This limit was imposed since, after removing E-S0 galaxies and AGNs, it splits the sample into two subsamples of similar numbers of galaxies. As is clear from the figures, the $\mathrm{H} \alpha$ growth curves do not seem to show any dependence on inclination, morphological type, and stellar mass. The only exception, for which a slight dependence is seen, is $x\left(\alpha_{\text {eff }}\right)$ when different inclinations are taken into account. In this case, the growth curves for edge-on and faceon galaxies look different, although still consistent with each other within the $1 \sigma$ limits shown in the figure.

\subsection{2. $H \alpha / H \beta$ Growth Curves}

Figure 6 shows $x\left(\alpha \beta_{50}\right)$ and $x$ ( $\left.\alpha \beta_{\text {eff }}\right)$ for different inclinations. Both curves are almost coincident irrespective of the inclination of the galaxies. It is remarkable that both $x\left(\alpha \beta_{50}\right)$ and $x\left(\alpha \beta_{\text {eff }}\right)$ show a very mild decline from the central regions to the outskirts, with a maximum value of $\approx 1.1$, which corresponds to an increase of $0.20 \mathrm{dex}$ in $c(\mathrm{H} \beta)$ or $0.43 \mathrm{mag}$ in $A_{V}$ at the center of the galaxies. This means that observing galaxies through a small aperture results in values of the extinction larger than those obtained with the integrated values of the $\mathrm{H} \alpha$ and $\mathrm{H} \beta$ fluxes. Figure 7 shows $x\left(\alpha \beta_{50}\right)$ and $x\left(\alpha \beta_{\text {eff }}\right)$ for different morphological types. $x\left(\alpha \beta_{50}\right)$ is slightly higher for $\mathrm{Sa}-\mathrm{Sbc}$ than for $\mathrm{Sc}-\mathrm{Sm}$ galaxies, reaching values of $\approx 1.15$ at the center of the galaxies in the first case. This difference is not observed for $x$ ( $\alpha \beta_{\text {eff }}$ ). Figure 8 shows $x\left(\alpha \beta_{50}\right)$ and $x\left(\alpha \beta_{\text {eff }}\right)$ for different stellar masses. As in the previous case, $x\left(\alpha \beta_{50}\right)$ is slightly higher for galaxies with $\log M^{*} / M_{\odot}>10.3$ than for galaxies with $\log M^{*} /$ $M_{\odot} \leqslant 10.3$ for small apertures, but this difference almost disappears in $x\left(\alpha \beta_{\text {eff }}\right)$. However, the differences observed in the median growth curves for galaxies of different morphological types and stellar masses are always lower than the dispersions around these median growth curves, which, as the figures show, increase as the radii of the apertures decrease.

This slight increase in the $\mathrm{H} \alpha / \mathrm{H} \beta$ growth curve observed for small apertures with respect to the integrated values can be compared with the variation in this Balmer line ratio as a function of electron temperature. Assuming case B 
Table 12

$x\left(\mathrm{~N} 2_{50}\right)$ for Two Bins of Morphological Types Assuming the Same Weight for All Galaxies

\begin{tabular}{|c|c|c|c|c|c|c|c|c|c|}
\hline \multirow{2}{*}{$r / R_{50}$} & \multicolumn{3}{|c|}{$\mathrm{Sa}-\mathrm{Sbc}$} & \multicolumn{3}{|c|}{ Sc-Sdm } & \multicolumn{3}{|c|}{ All } \\
\hline & $-\sigma$ & $x\left(\mathrm{~N} 2_{50}\right)$ & $+\sigma$ & $-\sigma$ & $x\left(\mathrm{~N} 2_{50}\right)$ & $+\sigma$ & $-\sigma$ & $x\left(\mathrm{~N} 2_{50}\right)$ & $+\sigma$ \\
\hline 0.00 & 0.005 & 0.075 & 0.249 & 0.031 & 0.087 & 0.152 & 0.006 & 0.081 & 0.221 \\
\hline 0.10 & 0.006 & 0.074 & 0.254 & 0.032 & 0.091 & 0.148 & 0.008 & 0.082 & 0.223 \\
\hline 0.20 & 0.006 & 0.074 & 0.255 & 0.033 & 0.090 & 0.151 & 0.008 & 0.082 & 0.223 \\
\hline 0.30 & 0.004 & 0.072 & 0.248 & 0.034 & 0.082 & 0.165 & 0.007 & 0.080 & 0.200 \\
\hline 0.40 & 0.001 & 0.072 & 0.226 & 0.034 & 0.072 & 0.163 & 0.004 & 0.072 & 0.189 \\
\hline 0.50 & 0.000 & 0.064 & 0.196 & 0.028 & 0.062 & 0.143 & 0.005 & 0.063 & 0.160 \\
\hline 0.80 & -0.008 & 0.036 & 0.127 & 0.010 & 0.051 & 0.087 & -0.000 & 0.039 & 0.114 \\
\hline 0.90 & -0.011 & 0.030 & 0.113 & 0.010 & 0.044 & 0.080 & -0.002 & 0.034 & 0.101 \\
\hline 1.00 & -0.010 & 0.025 & 0.102 & 0.006 & 0.042 & 0.075 & 0.002 & 0.031 & 0.090 \\
\hline 1.10 & -0.005 & 0.024 & 0.092 & 0.004 & 0.043 & 0.070 & 0.002 & 0.028 & 0.077 \\
\hline 1.20 & -0.005 & 0.022 & 0.083 & 0.003 & 0.039 & 0.068 & -0.002 & 0.025 & 0.072 \\
\hline 1.30 & -0.004 & 0.019 & 0.070 & 0.001 & 0.032 & 0.067 & -0.002 & 0.022 & 0.069 \\
\hline 1.80 & -0.002 & 0.009 & 0.023 & -0.001 & 0.008 & 0.030 & -0.002 & 0.008 & 0.024 \\
\hline 1.90 & -0.001 & 0.007 & 0.019 & -0.000 & 0.007 & 0.026 & -0.001 & 0.007 & 0.021 \\
\hline 2.00 & -0.002 & 0.005 & 0.017 & -0.004 & 0.006 & 0.021 & -0.003 & 0.006 & 0.019 \\
\hline 2.10 & -0.004 & 0.004 & 0.014 & -0.005 & 0.005 & 0.019 & -0.004 & 0.005 & 0.014 \\
\hline 2.20 & -0.002 & 0.003 & 0.010 & -0.004 & 0.003 & 0.017 & -0.003 & 0.003 & 0.011 \\
\hline 2.30 & -0.002 & 0.002 & 0.008 & -0.003 & 0.002 & 0.009 & -0.002 & 0.002 & 0.008 \\
\hline 2.40 & -0.001 & 0.001 & 0.004 & -0.001 & 0.001 & 0.003 & -0.001 & 0.001 & 0.004 \\
\hline 2.50 & 0.000 & 0.000 & 0.000 & 0.000 & 0.000 & 0.000 & 0.000 & 0.000 & 0.000 \\
\hline
\end{tabular}

Note. (1) Aperture radius in units of $R_{50}$. (2)-(4) Values of $x\left(\mathrm{~N}_{50}\right)$ corresponding to $15.86 \%, 50 \%$, and $84.14 \%$ of the distribution for Sa-Sbc galaxies. (5)-(7) Values of $x(\mathrm{~N} 250)$ corresponding to $15.86 \%, 50 \%$, and $84.14 \%$ of the distribution for Sc-Sdm galaxies. (8)-(10) Values of $x$ (N2 50 ) corresponding to $15.86 \%$, 50\%, and $84.14 \%$ of the distribution for all galaxies.

Table 13

$x\left(\mathrm{~N} 2_{\text {eff }}\right)$ for Two Bins of Morphological Types Assuming the Same Weight for All Galaxies

\begin{tabular}{|c|c|c|c|c|c|c|c|c|c|}
\hline \multirow{2}{*}{$r / R_{\mathrm{eff}}$} & \multicolumn{3}{|c|}{$\mathrm{Sa}-\mathrm{Sbc}$} & \multicolumn{3}{|c|}{$\mathrm{Sc}-\mathrm{Sdm}$} & \multicolumn{3}{|c|}{ All } \\
\hline & $-\sigma$ & $x\left(\mathrm{~N} 2_{\mathrm{eff}}\right)$ & $+\sigma$ & $-\sigma$ & $x\left(\mathrm{~N} 2_{\mathrm{eff}}\right)$ & $+\sigma$ & $-\sigma$ & $x\left(\mathrm{~N} 2_{\text {eff }}\right)$ & $+\sigma$ \\
\hline 0.00 & 0.008 & 0.073 & 0.252 & 0.004 & 0.066 & 0.149 & 0.006 & 0.072 & 0.170 \\
\hline 0.10 & 0.007 & 0.076 & 0.259 & 0.005 & 0.074 & 0.150 & 0.006 & 0.075 & 0.178 \\
\hline 0.20 & 0.008 & 0.068 & 0.236 & 0.001 & 0.062 & 0.141 & 0.005 & 0.064 & 0.171 \\
\hline 0.30 & 0.002 & 0.052 & 0.169 & -0.005 & 0.049 & 0.120 & 0.001 & 0.051 & 0.150 \\
\hline 0.40 & -0.006 & 0.045 & 0.127 & -0.007 & 0.044 & 0.093 & -0.006 & 0.044 & 0.104 \\
\hline 0.50 & -0.010 & 0.032 & 0.102 & -0.004 & 0.039 & 0.083 & -0.008 & 0.033 & 0.092 \\
\hline 0.60 & -0.010 & 0.023 & 0.074 & 0.001 & 0.036 & 0.073 & -0.007 & 0.029 & 0.074 \\
\hline 0.70 & -0.009 & 0.020 & 0.063 & 0.001 & 0.030 & 0.065 & -0.008 & 0.021 & 0.064 \\
\hline 0.80 & -0.008 & 0.017 & 0.048 & -0.007 & 0.022 & 0.056 & -0.007 & 0.017 & 0.051 \\
\hline 0.90 & -0.008 & 0.016 & 0.034 & -0.008 & 0.018 & 0.054 & -0.008 & 0.016 & 0.041 \\
\hline 1.00 & -0.005 & 0.012 & 0.030 & -0.008 & 0.013 & 0.041 & -0.006 & 0.013 & 0.032 \\
\hline 1.10 & -0.003 & 0.009 & 0.024 & -0.005 & 0.009 & 0.035 & -0.004 & 0.009 & 0.026 \\
\hline 1.20 & -0.004 & 0.005 & 0.017 & -0.003 & 0.006 & 0.022 & -0.003 & 0.006 & 0.017 \\
\hline 1.30 & -0.002 & 0.003 & 0.010 & -0.001 & 0.003 & 0.014 & -0.002 & 0.003 & 0.012 \\
\hline 1.40 & -0.001 & 0.002 & 0.006 & -0.001 & 0.002 & 0.007 & -0.001 & 0.002 & 0.006 \\
\hline 1.50 & 0.000 & 0.000 & 0.000 & 0.000 & 0.000 & 0.000 & 0.000 & 0.000 & 0.000 \\
\hline
\end{tabular}

Note. (1) Aperture radius in units of $R_{\text {eff }}$. (2)-(4) Values of $x(\mathrm{~N} 2$ eff $)$ corresponding to $15.86 \%, 50 \%$, and $84.14 \%$ of the Distribution for Sa-Sbc galaxies. (5)-(7) Values of $x\left(\mathrm{~N} 2_{\text {eff }}\right)$ corresponding to $15.86 \%, 50 \%$, and $84.14 \%$ of the distribution for Sc-Sdm galaxies. (8)-(10) Values of $x$ (N2 eff $)$ corresponding to $15.86 \%$, $50 \%$, and $84.14 \%$ of the distribution for all galaxies.

recombination and low-density limit, a substantial change, from 20,000 to $5000 \mathrm{~K}$, in electron temperature should be present to see a $10 \%$ change in $\mathrm{H} \alpha / \mathrm{H} \beta$. This change in electronic temperature is not observed in typical $\mathrm{H}$ II regions in the disks of spirals (Pérez-Montero \& Contini 2009). For this reason, a radial gradient on the dust content of star-forming 
Table 14

$x\left(\mathrm{~N} 2_{50}\right)$ for Two Bins of Stellar Masses Assuming the Same Weight for All Galaxies

\begin{tabular}{|c|c|c|c|c|c|c|}
\hline \multirow{2}{*}{$r / R_{50}$} & \multicolumn{3}{|c|}{$\log M^{*} \leqslant 10.3$} & \multicolumn{3}{|c|}{$\log M^{*}>10.3$} \\
\hline & $-\sigma$ & $x\left(\mathrm{~N} 2_{50}\right)$ & $+\sigma$ & $-\sigma$ & $x\left(\mathrm{~N} 2_{50}\right)$ & $+\sigma$ \\
\hline 0.00 & 0.006 & 0.075 & 0.145 & 0.013 & 0.081 & 0.258 \\
\hline 0.10 & 0.003 & 0.076 & 0.147 & 0.014 & 0.085 & 0.266 \\
\hline 0.20 & 0.004 & 0.076 & 0.146 & 0.014 & 0.083 & 0.269 \\
\hline 0.30 & 0.004 & 0.075 & 0.141 & 0.014 & 0.084 & 0.264 \\
\hline 0.40 & 0.008 & 0.064 & 0.132 & 0.002 & 0.083 & 0.249 \\
\hline 0.50 & 0.005 & 0.057 & 0.129 & 0.004 & 0.071 & 0.218 \\
\hline 0.60 & 0.004 & 0.047 & 0.120 & 0.008 & 0.059 & 0.191 \\
\hline 0.70 & -0.003 & 0.041 & 0.096 & 0.007 & 0.050 & 0.167 \\
\hline 0.80 & -0.002 & 0.038 & 0.088 & 0.003 & 0.042 & 0.138 \\
\hline 0.90 & -0.003 & 0.035 & 0.080 & 0.002 & 0.033 & 0.122 \\
\hline 1.00 & 0.001 & 0.033 & 0.072 & 0.002 & 0.031 & 0.111 \\
\hline 1.10 & 0.001 & 0.030 & 0.065 & 0.001 & 0.027 & 0.099 \\
\hline 1.20 & 0.001 & 0.027 & 0.063 & -0.007 & 0.023 & 0.083 \\
\hline 1.30 & -0.000 & 0.023 & 0.057 & -0.006 & 0.020 & 0.069 \\
\hline 1.40 & -0.001 & 0.019 & 0.052 & -0.005 & 0.018 & 0.059 \\
\hline 1.50 & -0.001 & 0.016 & 0.051 & -0.006 & 0.017 & 0.050 \\
\hline 1.60 & -0.001 & 0.013 & 0.041 & -0.001 & 0.015 & 0.042 \\
\hline 1.70 & -0.002 & 0.010 & 0.033 & -0.005 & 0.013 & 0.035 \\
\hline 1.80 & -0.001 & 0.008 & 0.024 & -0.004 & 0.010 & 0.029 \\
\hline 1.90 & -0.001 & 0.006 & 0.020 & -0.002 & 0.008 & 0.024 \\
\hline 2.00 & -0.002 & 0.006 & 0.016 & -0.003 & 0.006 & 0.019 \\
\hline 2.10 & -0.003 & 0.005 & 0.013 & -0.005 & 0.004 & 0.016 \\
\hline 2.20 & -0.002 & 0.003 & 0.010 & -0.003 & 0.003 & 0.012 \\
\hline 2.30 & -0.002 & 0.002 & 0.007 & -0.002 & 0.002 & 0.008 \\
\hline 2.40 & -0.001 & 0.001 & 0.004 & -0.001 & 0.001 & 0.004 \\
\hline 2.50 & 0.000 & 0.000 & 0.000 & 0.000 & 0.000 & 0.000 \\
\hline
\end{tabular}

Note. (1) Aperture radius in units of $R_{50}$. (2)-(4) Values of $x\left(\mathrm{~N} 2_{50}\right)$ corresponding to $15.86 \%, 50 \%$, and $84.14 \%$ of the distribution for $\log M^{*} \leqslant 10.3$ galaxies. (5)(7) Values of $x(\mathrm{~N} 250)$ corresponding to $15.86 \%, 50 \%$, and $84.14 \%$ of the distribution for $\log M^{*}>10.3$ galaxies.

Table 15

$x\left(\mathrm{~N} 2_{\text {eff }}\right)$ for Two Bins of Stellar Masses Assuming the Same Weight for All Galaxies

\begin{tabular}{lrcccccc}
\hline \hline \multirow{2}{*}{$r / R_{\text {eff }}$} & \multicolumn{3}{c}{$\log M^{*} \leqslant 10.3$} & & \multicolumn{3}{c}{$\log M^{*}>10.3$} \\
\cline { 2 - 3 } \cline { 6 - 7 } & \multicolumn{1}{c}{$-\sigma$} & $x\left(\mathrm{~N} 2_{\text {eff }}\right)$ & $+\sigma$ & & $-\sigma$ & $x\left(\mathrm{~N} 2_{\text {eff }}\right)$ & $+\sigma$ \\
\hline 0.00 & 0.003 & 0.068 & 0.148 & & 0.009 & 0.077 & 0.293 \\
0.10 & 0.003 & 0.073 & 0.151 & & 0.007 & 0.078 & 0.300 \\
0.20 & 0.004 & 0.058 & 0.134 & & 0.008 & 0.077 & 0.249 \\
0.30 & 0.000 & 0.049 & 0.113 & & -0.000 & 0.056 & 0.188 \\
0.40 & -0.005 & 0.044 & 0.092 & & -0.008 & 0.042 & 0.131 \\
0.50 & -0.002 & 0.039 & 0.085 & & -0.012 & 0.029 & 0.101 \\
0.60 & 0.003 & 0.033 & 0.074 & & -0.012 & 0.020 & 0.074 \\
0.70 & 0.003 & 0.030 & 0.065 & & -0.012 & 0.019 & 0.064 \\
0.80 & -0.002 & 0.022 & 0.055 & & -0.010 & 0.015 & 0.048 \\
0.90 & -0.005 & 0.017 & 0.051 & & -0.009 & 0.013 & 0.031 \\
1.00 & -0.003 & 0.015 & 0.039 & & -0.007 & 0.012 & 0.026 \\
1.10 & -0.001 & 0.010 & 0.034 & & -0.006 & 0.008 & 0.019 \\
1.20 & -0.002 & 0.006 & 0.023 & & -0.005 & 0.005 & 0.014 \\
1.30 & -0.001 & 0.004 & 0.016 & & -0.003 & 0.002 & 0.008 \\
1.40 & -0.001 & 0.002 & 0.007 & & -0.001 & 0.001 & 0.005 \\
1.50 & 0.000 & 0.000 & 0.000 & & 0.000 & 0.000 & 0.000 \\
\hline
\end{tabular}

Note. (1) Aperture radius in units of $R_{\text {eff. }}$ (2)-(4) Values of $x\left(\mathrm{~N} 2_{\text {eff }}\right)$ corresponding to $15.86 \%, 50 \%$, and $84.14 \%$ of the distribution for edge-on $\log M^{*} \leqslant 10.3$ galaxies. (5)-(7) Values of $x$ ( $\mathrm{N} 2_{\text {eff }}$ ) corresponding to $15.86 \%$, $50 \%$, and $84.14 \%$ of the distribution for face-on $\log M^{*}>10.3$ galaxies. regions, likely related to the abundance gradient measured in spiral galaxies, would explain this aperture effect.

\subsubsection{N2 Growth Curves}

Figure 9 shows $x\left(\mathrm{~N} 2_{50}\right)$ and $x\left(\mathrm{~N} 2_{\text {eff }}\right)$ for different inclinations. $x\left(\mathrm{~N} 2_{50}\right)$ and $x\left(\mathrm{~N} 2_{\text {eff }}\right)$ behave in a similar way to $x\left(\alpha \beta_{50}\right)$ and $x\left(\alpha \beta_{\text {eff }}\right)$, that is, they show a mild decline from the inner parts of the galaxies and do not show clear differences related to the inclination of galaxies. $x\left(\mathrm{~N} 2_{50}\right)$ and $x\left(\mathrm{~N} 2_{\text {eff }}\right)$ show central values that reach maxima of $\approx 1.2-1.3$ and larger dispersions around these central values in the inner regions of the galaxies compared to the outskirts. The meaning of this trend is that observing galaxies through small apertures results in values of $\mathrm{N} 2$ larger than the ones obtained with the integrated values. Figure 10 shows $x\left(\mathrm{~N} 2_{50}\right)$ and $x\left(\mathrm{~N} 2_{\mathrm{eff}}\right)$ for different morphological types. The behavior of $x\left(\mathrm{~N} 2_{50}\right)$ and $x\left(\mathrm{~N} 2_{\text {eff }}\right)$ is similar to the one previously described. Figure 11 shows $x\left(\mathrm{~N} 2_{50}\right)$ and $x\left(\mathrm{~N} 2_{\text {eff }}\right)$ for different stellar masses, which shows a marginal difference for small apertures with more massive galaxies showing larger values of $x\left(\mathrm{~N} 2{ }_{50}\right)$ and $x\left(\mathrm{~N} 2_{\text {eff }}\right)$ than less massive ones. However, again this difference is much smaller than the dispersions around the median values of $x\left(\mathrm{~N} 2_{50}\right)$ and $x\left(\mathrm{~N} 2_{\text {eff }}\right)$. As we will show in the next section, the radial change of the $\mathrm{N} 2$ growth curves reflects a radial change in the oxygen abundances.

\subsubsection{O3N2 Growth Curves}

Figure 12 shows $x\left(\mathrm{O} 3 \mathrm{~N} 2{ }_{50}\right)$ and $x\left(\mathrm{O} 3 \mathrm{~N} 2_{\mathrm{eff}}\right)$ for different inclinations. Following this figure, $x\left(\mathrm{O} 3 \mathrm{~N} 2{ }_{50}\right)$ and $x\left(\mathrm{O} 3 \mathrm{~N} 2_{\mathrm{eff}}\right)$ show an almost linear increasing trend from the inner regions to the outskirts of the galaxies, and no difference with the inclination of the galaxy is apparent. This means that observing galaxies through small apertures results in values of $\mathrm{O} 3 \mathrm{~N} 2$ smaller than those obtained with the integrated values. Figure 13 shows $x\left(\mathrm{O} 3 \mathrm{~N} 2{ }_{50}\right)$ and $x\left(\mathrm{O} 3 \mathrm{~N} 2{ }_{\text {eff }}\right)$ for different morphological types. In this case, $\mathrm{Sa}-\mathrm{Sbc}$ galaxies show larger values of $x\left(\mathrm{O} 3 \mathrm{~N} 2{ }_{50}\right)$ and $x\left(\mathrm{O} 3 \mathrm{~N} 2{ }_{\mathrm{eff}}\right)$ for apertures with $R / R_{50} \leqslant 1.2$ and $R / R_{\text {eff }} \leqslant 0.7$, respectively, than $\mathrm{Sc}-\mathrm{Sm}$ galaxies, although the difference is lower than the dispersions around the median values. Figure 14 shows $x\left(\mathrm{O} 3 \mathrm{~N} 2_{50}\right)$ and $x\left(\mathrm{O} 3 \mathrm{~N} 2_{\mathrm{eff}}\right)$ for different stellar masses. Similarly to the previous case, galaxies with $\log M^{*} / M_{\odot}>10.3$ present larger values of $x\left(\mathrm{O} 3 \mathrm{~N} 2_{50}\right)$ and $x\left(\mathrm{O} 3 \mathrm{~N} 2{ }_{\mathrm{eff}}\right)$ for apertures with $R / R_{50} \leqslant 1.2$ and $R / R_{\mathrm{eff}} \leqslant 0.7$, respectively, than galaxies with $\log M^{*} / M_{\odot} \leqslant 10.3$, but as commented above, this difference is smaller than the dispersions around the median values of $x\left(\mathrm{O} 3 \mathrm{~N} 22_{50}\right)$ and $x\left(\mathrm{O} 3 \mathrm{~N} 22_{\mathrm{eff}}\right)$. In this case also the radial change of the $\mathrm{O} 3 \mathrm{~N} 2$ growth curves can be interpreted in terms of a radial change of the oxygen abundanes.

Tables 4-19 show the numerical values of the median growth curves and the $1 \sigma$ confidence intervals corresponding to the growth curves previously shown. These tables also contain the fits to all spiral galaxies considered in this work.

As a summary of the present section we show in Table 20 the coefficients of the fits to fifth-order polynomials of the aperture corrections previously discussed.

\subsection{Corrections for Fixed Physical Apertures}

The large field of view covered by the CALIFA data allows us to predict the fraction of flux enclosed within a given angular aperture with respect to the flux enclosed within a fixed 
Table 16

$x\left(\mathrm{O} 3 \mathrm{~N}_{50}\right)$ for Two Bins of Morphological Types Assuming the Same Weight for All Galaxies

\begin{tabular}{|c|c|c|c|c|c|c|c|c|c|}
\hline \multirow{2}{*}{$r / R_{50}$} & \multicolumn{3}{|c|}{$\mathrm{Sa}-\mathrm{Sbc}$} & \multicolumn{3}{|c|}{$\mathrm{Sc}-\mathrm{Sdm}$} & \multicolumn{3}{|c|}{ All } \\
\hline & $-\sigma$ & $x\left(\mathrm{O} 3 \mathrm{~N} 2_{50}\right)$ & $+\sigma$ & $-\sigma$ & $x\left(\mathrm{O} 3 \mathrm{~N} 2{ }_{50}\right)$ & $+\sigma$ & $-\sigma$ & $x\left(\mathrm{O} 3 \mathrm{~N} 2{ }_{50}\right)$ & $+\sigma$ \\
\hline 0.00 & -0.275 & -0.091 & 0.083 & -0.459 & -0.234 & -0.084 & -0.368 & -0.127 & 0.063 \\
\hline 0.20 & -0.274 & -0.095 & 0.092 & -0.465 & -0.250 & -0.080 & -0.379 & -0.128 & 0.060 \\
\hline 0.30 & -0.268 & -0.081 & 0.085 & -0.448 & -0.225 & -0.088 & -0.348 & -0.123 & 0.063 \\
\hline 0.40 & -0.241 & -0.089 & 0.077 & -0.415 & -0.196 & -0.100 & -0.299 & -0.125 & 0.061 \\
\hline 0.50 & -0.217 & -0.080 & 0.078 & -0.371 & -0.199 & -0.066 & -0.251 & -0.111 & 0.052 \\
\hline 0.80 & -0.193 & -0.060 & 0.038 & -0.280 & -0.133 & -0.034 & -0.208 & -0.078 & 0.022 \\
\hline 0.90 & -0.182 & -0.067 & 0.033 & -0.252 & -0.118 & -0.038 & -0.208 & -0.074 & 0.019 \\
\hline 1.00 & -0.175 & -0.062 & 0.024 & -0.222 & -0.102 & -0.053 & -0.195 & -0.069 & 0.013 \\
\hline 1.10 & -0.166 & -0.058 & 0.023 & -0.206 & -0.093 & -0.052 & -0.182 & -0.073 & 0.008 \\
\hline 1.20 & -0.153 & -0.055 & 0.018 & -0.188 & -0.089 & -0.051 & -0.167 & -0.069 & -0.002 \\
\hline 1.30 & -0.139 & -0.049 & 0.009 & -0.171 & -0.070 & -0.040 & -0.156 & -0.061 & -0.003 \\
\hline 1.80 & -0.077 & -0.029 & 0.002 & -0.075 & -0.027 & -0.001 & -0.075 & -0.028 & 0.001 \\
\hline 1.90 & -0.067 & -0.025 & 0.004 & -0.068 & -0.019 & -0.001 & -0.068 & -0.024 & 0.003 \\
\hline 2.00 & -0.055 & -0.021 & 0.005 & -0.063 & -0.009 & -0.001 & -0.057 & -0.020 & 0.004 \\
\hline 2.10 & -0.046 & -0.017 & 0.003 & -0.054 & -0.006 & 0.002 & -0.048 & -0.016 & 0.003 \\
\hline 2.20 & -0.040 & -0.011 & 0.003 & -0.042 & -0.007 & 0.003 & -0.040 & -0.011 & 0.003 \\
\hline 2.30 & -0.029 & -0.008 & 0.005 & -0.025 & -0.007 & 0.004 & -0.026 & -0.008 & 0.004 \\
\hline 2.40 & -0.013 & -0.005 & 0.001 & -0.012 & -0.004 & 0.002 & -0.013 & -0.004 & 0.002 \\
\hline 2.50 & 0.000 & 0.000 & 0.000 & 0.000 & 0.000 & 0.000 & 0.000 & 0.000 & 0.000 \\
\hline
\end{tabular}

Note. (1) Aperture radius in units of $R_{50}$. (2)-(4) Values of $x\left(\mathrm{O} 3 \mathrm{~N} 2_{50}\right)$ corresponding to $15.86 \%, 50 \%$, and $84.14 \%$ of the Distribution for Sa-Sbc galaxies. (5)-(7) Values of $x\left(\mathrm{O} 3 \mathrm{~N} 2_{50}\right)$ corresponding to $15.86 \%, 50 \%$, and $84.14 \%$ of the distribution for Sc-Sdm galaxies. (8)-(10) Values of $x\left(\mathrm{O} 3 \mathrm{~N} 2_{50}\right)$ corresponding to $15.86 \%$, $50 \%$, and $84.14 \%$ of the distribution for all galaxies.

Table 17

$x\left(\mathrm{O} 3 \mathrm{~N} 2_{\text {eff }}\right)$ for Two Bins of Morphological Types Assuming the Same Weight for All Galaxies

\begin{tabular}{|c|c|c|c|c|c|c|c|c|c|}
\hline \multirow{2}{*}{$r / R_{\text {eff }}$} & \multicolumn{3}{|c|}{$\mathrm{Sa}-\mathrm{Sbc}$} & \multicolumn{3}{|c|}{ Sc-Sdm } & \multicolumn{3}{|c|}{ All } \\
\hline & $-\sigma$ & $x\left(\mathrm{O} 3 \mathrm{~N} 2_{\mathrm{eff}}\right)$ & $+\sigma$ & $-\sigma$ & $x\left(\mathrm{O} 3 \mathrm{~N} 2_{\mathrm{eff}}\right)$ & $+\sigma$ & $-\sigma$ & $x\left(\mathrm{O} 3 \mathrm{~N} 2_{\mathrm{eff}}\right)$ & $+\sigma$ \\
\hline 0.00 & -0.291 & -0.092 & 0.154 & -0.434 & -0.214 & -0.082 & -0.338 & -0.140 & 0.072 \\
\hline 0.10 & -0.300 & -0.093 & 0.157 & -0.452 & -0.224 & -0.081 & -0.355 & -0.145 & 0.071 \\
\hline 0.20 & -0.278 & -0.079 & 0.146 & -0.422 & -0.204 & -0.085 & -0.310 & -0.137 & 0.081 \\
\hline 0.30 & -0.258 & -0.093 & 0.103 & -0.396 & -0.186 & -0.079 & -0.264 & -0.117 & 0.074 \\
\hline 0.40 & -0.229 & -0.067 & 0.093 & -0.324 & -0.155 & -0.058 & -0.234 & -0.101 & 0.020 \\
\hline 0.50 & -0.201 & -0.065 & 0.051 & -0.278 & -0.123 & -0.061 & -0.213 & -0.091 & 0.009 \\
\hline 0.60 & -0.189 & -0.063 & 0.057 & -0.219 & -0.095 & -0.057 & -0.192 & -0.081 & -0.016 \\
\hline 0.70 & -0.166 & -0.070 & 0.025 & -0.184 & -0.086 & -0.043 & -0.172 & -0.079 & -0.001 \\
\hline 0.80 & -0.152 & -0.070 & 0.004 & -0.153 & -0.081 & -0.014 & -0.152 & -0.070 & 0.003 \\
\hline 0.90 & -0.123 & -0.067 & 0.014 & -0.120 & -0.063 & 0.005 & -0.123 & -0.065 & 0.010 \\
\hline 1.00 & -0.104 & -0.056 & 0.015 & -0.104 & -0.048 & -0.007 & -0.102 & -0.050 & 0.005 \\
\hline 1.10 & -0.088 & -0.038 & 0.011 & -0.077 & -0.028 & -0.008 & -0.085 & -0.035 & 0.000 \\
\hline 1.20 & -0.058 & -0.028 & 0.001 & -0.047 & -0.023 & -0.005 & -0.057 & -0.024 & -0.003 \\
\hline 1.30 & -0.034 & -0.015 & 0.008 & -0.051 & -0.011 & 0.000 & -0.036 & -0.014 & 0.004 \\
\hline 1.40 & -0.020 & -0.007 & 0.014 & -0.016 & -0.006 & 0.001 & -0.020 & -0.006 & 0.007 \\
\hline 1.50 & 0.000 & 0.000 & 0.000 & 0.000 & 0.000 & 0.000 & 0.000 & 0.000 & 0.000 \\
\hline
\end{tabular}

Note. (1) Aperture radius in units of $R_{\text {eff. }}$ (2)-(4) Values of $x\left(\mathrm{O} 3 \mathrm{~N} 2_{\text {eff }}\right)$ corresponding to $15.86 \%, 50 \%$, and $84.14 \%$ of the distribution for $\mathrm{Sa}-\mathrm{Sbc}$ galaxies. (5)-(7) Values of $x\left(\mathrm{O} 3 \mathrm{~N} 22_{\text {eff }}\right)$ corresponding to $15.86 \%, 50 \%$, and $84.14 \%$ of the distribution for Sc-Sdm galaxies. (8)-(10) Values of $x(\mathrm{O} 3 \mathrm{~N} 2$ eff $)$ corresponding to $15.86 \%$, $50 \%$, and $84.14 \%$ of the distribution for all galaxies.

physicalaperture at different redshifts. In our case, we have focused on the SDSS and SAMI fields of view. As the median value of $R_{\text {eff }}$ of our sample (spirals from Sa to Sdm excluding
AGNs) is $\approx 6.68 \mathrm{kpc}$, and based on $x$ ( $\left.\alpha_{\text {eff }}\right)$ shown in Figure 2, we estimate the quantities of interest within two circular apertures, one containing most of the flux of the galaxy $(10 \mathrm{kpc}$ radius, 
Table 18

$x\left(\mathrm{O} 3 \mathrm{~N}_{20}\right)$ for Two Bins of Stellar Masses Assuming the Same Weight for All Galaxies

\begin{tabular}{|c|c|c|c|c|c|c|}
\hline \multirow{2}{*}{$r / R_{50}$} & \multicolumn{3}{|c|}{$\log M^{*} \leqslant 10.3$} & \multicolumn{3}{|c|}{$\log M^{*}>10.3$} \\
\hline & $-\sigma$ & $x\left(\mathrm{O} 3 \mathrm{~N} 2{ }_{50}\right)$ & $+\sigma$ & $-\sigma$ & $x\left(\mathrm{O} 3 \mathrm{~N} 2_{50}\right)$ & $+\sigma$ \\
\hline 0.00 & -0.445 & -0.228 & -0.024 & -0.203 & -0.055 & 0.139 \\
\hline 0.10 & -0.459 & -0.237 & -0.021 & -0.209 & -0.059 & 0.146 \\
\hline 0.20 & -0.462 & -0.232 & -0.022 & -0.204 & -0.059 & 0.144 \\
\hline 0.30 & -0.437 & -0.215 & -0.004 & -0.199 & -0.050 & 0.134 \\
\hline 0.40 & -0.403 & -0.196 & -0.018 & -0.196 & -0.057 & 0.120 \\
\hline 0.50 & -0.362 & -0.188 & -0.042 & -0.187 & -0.038 & 0.117 \\
\hline 0.60 & -0.333 & -0.157 & -0.023 & -0.173 & -0.026 & 0.087 \\
\hline 0.70 & -0.307 & -0.131 & -0.010 & -0.179 & -0.037 & 0.074 \\
\hline 0.80 & -0.283 & -0.111 & -0.027 & -0.186 & -0.044 & 0.044 \\
\hline 0.90 & -0.255 & -0.107 & -0.031 & -0.172 & -0.045 & 0.042 \\
\hline 1.00 & -0.221 & -0.097 & -0.028 & -0.158 & -0.055 & 0.027 \\
\hline 1.10 & -0.204 & -0.089 & -0.024 & -0.153 & -0.051 & 0.024 \\
\hline 1.20 & -0.190 & -0.078 & -0.026 & -0.151 & -0.049 & 0.018 \\
\hline 1.30 & -0.174 & -0.065 & -0.029 & -0.135 & -0.047 & 0.010 \\
\hline 1.40 & -0.148 & -0.055 & -0.025 & -0.121 & -0.046 & 0.007 \\
\hline 1.50 & -0.125 & -0.049 & -0.013 & -0.108 & -0.045 & 0.008 \\
\hline 1.60 & -0.111 & -0.043 & -0.006 & -0.095 & -0.039 & 0.009 \\
\hline 1.70 & -0.100 & -0.040 & -0.006 & -0.084 & -0.032 & 0.012 \\
\hline 1.80 & -0.086 & -0.030 & -0.010 & -0.074 & -0.026 & 0.005 \\
\hline 1.90 & -0.073 & -0.028 & -0.004 & -0.062 & -0.021 & 0.006 \\
\hline 2.00 & -0.065 & -0.022 & -0.001 & -0.050 & -0.017 & 0.006 \\
\hline 2.10 & -0.052 & -0.017 & 0.003 & -0.044 & -0.015 & 0.003 \\
\hline 2.20 & -0.042 & -0.011 & 0.002 & -0.039 & -0.010 & 0.004 \\
\hline 2.30 & -0.026 & -0.007 & 0.003 & -0.030 & -0.008 & 0.006 \\
\hline 2.40 & -0.013 & -0.004 & 0.002 & -0.014 & -0.005 & 0.001 \\
\hline 2.50 & 0.000 & 0.000 & 0.000 & 0.000 & 0.000 & 0.000 \\
\hline
\end{tabular}

Note. (1) Aperture radius in units of $R_{50}$. (2)-(4) Values of $x\left(\mathrm{O} 3 \mathrm{~N} 2_{50}\right)$ corresponding to $15.86 \%, 50 \%$, and $84.14 \%$ of the distribution for log $M^{*} \leqslant 10.3$ galaxies. (5)-(7) Values of $x(\mathrm{O} 3 \mathrm{~N} 250)$ corresponding to $15.86 \%, 50 \%$, and $84.14 \%$ of the distribution for $\log M^{*}>10.3$ galaxies.

Table 19

$x\left(\mathrm{O} 3 \mathrm{~N} 2_{\mathrm{eff}}\right)$ for Two Bins of Stellar Masses Assuming the Same Weight for All Galaxies

\begin{tabular}{|c|c|c|c|c|c|c|}
\hline \multirow{2}{*}{$r / R_{\mathrm{eff}}$} & \multicolumn{3}{|c|}{$\log M^{*} \leqslant 10.3$} & \multicolumn{3}{|c|}{$\log M^{*}>10.3$} \\
\hline & $-\sigma$ & $x\left(\mathrm{O} 3 \mathrm{~N} 2_{\mathrm{eff}}\right)$ & $+\sigma$ & $-\sigma$ & $x\left(\mathrm{O} 3 \mathrm{~N} 2_{\mathrm{eff}}\right)$ & $+\sigma$ \\
\hline 0.00 & -0.407 & -0.215 & 0.005 & -0.177 & -0.059 & 0.171 \\
\hline 0.10 & -0.427 & -0.236 & 0.008 & -0.189 & -0.064 & 0.178 \\
\hline 0.20 & -0.407 & -0.211 & 0.004 & -0.177 & -0.058 & 0.176 \\
\hline 0.30 & -0.347 & -0.185 & 0.009 & -0.161 & -0.053 & 0.121 \\
\hline 0.40 & -0.314 & -0.151 & -0.027 & -0.173 & -0.054 & 0.098 \\
\hline 0.50 & -0.276 & -0.125 & -0.032 & -0.157 & -0.050 & 0.073 \\
\hline 0.60 & -0.221 & -0.102 & -0.041 & -0.169 & -0.058 & 0.058 \\
\hline 0.70 & -0.206 & -0.098 & -0.025 & -0.159 & -0.064 & 0.027 \\
\hline 0.80 & -0.157 & -0.088 & 0.002 & -0.130 & -0.063 & 0.004 \\
\hline 0.90 & -0.124 & -0.068 & 0.014 & -0.111 & -0.044 & 0.004 \\
\hline 1.00 & -0.121 & -0.053 & 0.004 & -0.094 & -0.042 & 0.012 \\
\hline 1.10 & -0.084 & -0.033 & -0.000 & -0.084 & -0.035 & -0.000 \\
\hline 1.20 & -0.056 & -0.024 & -0.003 & -0.059 & -0.029 & -0.002 \\
\hline 1.30 & -0.045 & -0.013 & 0.004 & -0.034 & -0.014 & 0.005 \\
\hline 1.40 & -0.018 & -0.007 & 0.009 & -0.020 & -0.005 & 0.006 \\
\hline 1.50 & 0.000 & 0.000 & 0.000 & 0.000 & 0.000 & 0.000 \\
\hline
\end{tabular}

Note. (1) Aperture radius in units of $R_{\text {eff. }}$ (2)-(4) Values of $x\left(\mathrm{O} 3 \mathrm{~N} 2_{\text {eff }}\right)$ corresponding to $15.86 \%, 50 \%$, and $84.14 \%$ of the distribution for $\log M^{*} \leqslant 10.3$ galaxies. (5)-(7) Values of $x\left(\mathrm{O} 3 \mathrm{~N} 22_{\text {eff }}\right)$ corresponding to $15.86 \%, 50 \%$, and $84.14 \%$ of the distribution for $\log M^{*}>10.3$ galaxies.

containing on average $90 \%$ of the total $\mathrm{H} \alpha$ flux) and another one enclosing the flux in the central region $(3.3 \mathrm{kpc}$ radius, containing on average $30 \%$ of the total $\mathrm{H} \alpha$ flux). Then for each of the CALIFA spirals we measure each of the relevant quantities within the circular aperture subtended by the SDSS fiber if the galaxy was placed at different redshifts. Figure 15 gives the median values of the aperture corrections with respect to an aperture of $10 \mathrm{kpc}$ radius for the $f(\mathrm{H} \alpha), f(\mathrm{H} \alpha) / f(\mathrm{H} \beta) 2$, and $\mathrm{O} 3 \mathrm{~N} 2$. The SDSS 
Table 20

Results of the Fits of the Fixed Angular Aperture Corrections given in Tables 4-19 to a Fifth-order Polynomial of the Type $a_{0}+a_{1} x+a_{2} x^{2}+a_{3} x^{3}+a_{4} x^{4}+a_{5} x^{5}$

\begin{tabular}{|c|c|c|c|c|c|c|c|}
\hline $\begin{array}{l}\text { Aperture } \\
\text { Correction }\end{array}$ & $\begin{array}{l}\text { Selection } \\
\text { Criterion }\end{array}$ & $a_{0}$ & $a_{1}$ & $a_{2}$ & $a_{3}$ & $a_{4}$ & $a_{5}$ \\
\hline \multirow[t]{5}{*}{$x\left(\alpha_{50}\right)$} & All & 0.0000 & 0.1599 & 0.5027 & -0.2276 & 0.0167 & 0.0037 \\
\hline & $\mathrm{Sa}-\mathrm{Sbc}$ & 0.0000 & 0.1588 & 0.5059 & -0.2649 & 0.0459 & -0.0022 \\
\hline & Sc-Sdm & 0.0000 & 0.1192 & 0.6799 & -0.3951 & 0.0812 & -0.0056 \\
\hline & $\log M^{*} / M_{\odot} \leqslant 10.3$ & 0.0000 & 0.1661 & 0.4937 & -0.2044 & 0.0024 & 0.0061 \\
\hline & $10.3 \leqslant \log M^{*} / M_{\odot}$ & 0.0000 & 0.1691 & 0.4630 & -0.1956 & 0.0079 & 0.0044 \\
\hline \multirow[t]{5}{*}{$x\left(\alpha \beta_{50}\right)$} & All & 1.1253 & 0.0274 & -0.3125 & 0.2959 & -0.1091 & 0.0143 \\
\hline & $\mathrm{Sa}-\mathrm{Sbc}$ & 1.1453 & 0.1087 & -0.4826 & 0.4127 & -0.1432 & 0.0179 \\
\hline & Sc-Sdm & 1.0912 & 0.0346 & -0.2795 & 0.2680 & -0.1026 & 0.0142 \\
\hline & $\log M^{*} / M_{\odot} \leqslant 10.3$ & 1.1053 & -0.0108 & -0.2144 & 0.2275 & -0.0916 & 0.0132 \\
\hline & $10.3 \leqslant \log M^{*} / M_{\odot}$ & 1.1801 & 0.0548 & -0.4810 & 0.4594 & -0.1721 & 0.0229 \\
\hline \multirow[t]{5}{*}{$x\left(\mathrm{~N} 2_{50}\right)$} & All & 0.0812 & 0.0477 & -0.2779 & 0.2715 & -0.1075 & 0.0153 \\
\hline & $\mathrm{Sa}-\mathrm{Sbc}$ & 0.0809 & 0.0843 & -0.3771 & 0.3646 & -0.1439 & 0.0204 \\
\hline & Sc-Sdm & 0.0923 & -0.0394 & -0.0350 & 0.0353 & -0.0137 & 0.0021 \\
\hline & $\log M^{*} / M_{\odot} \leqslant 10.3$ & 0.0769 & 0.0129 & -0.1737 & 0.1749 & -0.0705 & 0.0102 \\
\hline & $10.3 \leqslant \log M^{*} / M_{\odot}$ & 0.1021 & 0.0944 & -0.4838 & 0.4857 & -0.1957 & 0.0281 \\
\hline \multirow[t]{5}{*}{$x\left(\mathrm{O} 3 \mathrm{~N} 2_{50}\right)$} & All & -0.1299 & -0.0366 & 0.2645 & -0.2624 & 0.1092 & -0.0164 \\
\hline & $\mathrm{Sa}-\mathrm{Sbc}$ & -0.0947 & 0.0003 & 0.1096 & -0.1220 & 0.0561 & -0.0090 \\
\hline & $\mathrm{Sc}-\mathrm{Sdm}$ & -0.2433 & -0.0711 & 0.5757 & -0.5465 & 0.2132 & -0.0304 \\
\hline & $\log M^{*} / M_{\odot} \leqslant 10.3$ & -0.2318 & -0.0840 & 0.6316 & -0.6166 & 0.2423 & -0.0342 \\
\hline & $10.3 \leqslant \log M^{*} / M_{\odot}$ & -0.0616 & 0.0597 & -0.0420 & -0.0329 & 0.0401 & -0.0090 \\
\hline \multirow[t]{5}{*}{$x\left(\alpha_{\text {eff }}\right)$} & All & 0.0000 & 0.2469 & 1.8195 & -1.9620 & 0.8182 & -0.1297 \\
\hline & $\mathrm{Sa}-\mathrm{Sbc}$ & 0.0000 & 0.2338 & 2.0857 & -2.6054 & 1.3520 & -0.2758 \\
\hline & Sc-Sdm & 0.0000 & 0.3275 & 1.0776 & -0.4843 & -0.2743 & 0.1458 \\
\hline & $\log M^{*} / M_{\odot} \leqslant 10.3$ & 0.0000 & 0.1685 & 2.4921 & -3.2276 & 1.7208 & -0.3527 \\
\hline & $10.3 \leqslant \log M^{*} / M_{\odot}$ & 0.0000 & 0.1504 & 2.0411 & -2.1685 & 0.9098 & -0.1455 \\
\hline \multirow{5}{*}{$\overline{x\left(\alpha \beta_{\text {eff }}\right)}$} & All & 1.1100 & 0.0648 & -0.8553 & 1.3690 & -0.8714 & 0.1986 \\
\hline & $\mathrm{Sa}-\mathrm{Sbc}$ & 1.1153 & 0.0497 & -0.6904 & 1.0995 & -0.7145 & 0.1672 \\
\hline & Sc-Sdm & 1.1027 & 0.0477 & -0.8742 & 1.5044 & -1.0126 & 0.2426 \\
\hline & $\log M^{*} / M_{\odot} \leqslant 10.3$ & 1.0977 & -0.0051 & -0.6129 & 1.1462 & -0.8099 & 0.2003 \\
\hline & $10.3 \leqslant \log M^{*} / M_{\odot}$ & 1.1474 & -0.0245 & -0.5907 & 0.8897 & -0.5065 & 0.1027 \\
\hline \multirow[t]{5}{*}{$x\left(\mathrm{~N} 2_{\text {eff }}\right)$} & All & 0.0729 & 0.0320 & -0.5459 & 0.9304 & -0.6278 & 0.1508 \\
\hline & $\mathrm{Sa}-\mathrm{Sbc}$ & 0.0771 & 0.0762 & -0.7109 & 1.1317 & -0.7310 & 0.1698 \\
\hline & Sc-Sdm & 0.0690 & 0.0123 & -0.3467 & 0.5777 & -0.3970 & 0.0991 \\
\hline & $\log M^{*} / M_{\odot} \leqslant 10.3$ & 0.0706 & -0.0218 & -0.2287 & 0.4115 & -0.2904 & 0.0735 \\
\hline & $10.3 \leqslant \log M^{*} / M_{\odot}$ & 0.0837 & 0.1841 & -1.3430 & 2.2246 & -1.4944 & 0.3581 \\
\hline \multirow[t]{5}{*}{$x\left(\mathrm{O} 3 \mathrm{~N} 2_{\mathrm{eff}}\right)$} & All & -0.1420 & -0.0977 & 1.0274 & -1.8753 & 1.4059 & -0.3702 \\
\hline & $\mathrm{Sa}-\mathrm{Sbc}$ & -0.0920 & -0.0559 & 0.6785 & -1.4892 & 1.2567 & -0.3538 \\
\hline & $\mathrm{Sc}-\mathrm{Sdm}$ & -0.2147 & -0.2356 & 1.8838 & -2.9887 & 1.9851 & -0.4784 \\
\hline & $\log M^{*} / M_{\odot} \leqslant 10.3$ & -0.2192 & -0.2647 & 2.1138 & -3.5033 & 2.4170 & -0.5995 \\
\hline & $10.3 \leqslant \log M^{*} / M_{\odot}$ & -0.0615 & 0.0185 & 0.0830 & -0.3739 & 0.4270 & -0.1386 \\
\hline
\end{tabular}

fiber covers an aperture of $\approx 10 \mathrm{kpc}$ at a redshift of $z \approx 0.6$. The trends with redshift shown by the aperture corrections are similar to the ones obtained in previous sections as a function of $R_{50}$ and $R_{\text {eff }}$. Figure 16 also provides corrections for a physical aperture of $3.3 \mathrm{kpc}$ radius. In this case, the SDSS fiber covers this aperture at a redshift of $z \approx 0.12$.

We also provide corrections with respect to these two fixed apertures for the SAMI case (a circular bundle of fibers of 15 arcsec diameter). The results are shown in Figures 17 and 18. The larger size of the SAMI aperture compared to the SDSS one reduces the range of applicability of our aperture corrections since it results in complete coverage of the 10 and $3.3 \mathrm{kpc}$ apertures at redshifts of $z \approx 0.07$ and 0.02 , respectively.
Tables 21-24 list the median values and 1- $\sigma$ dispersions of the aperture corrections for fixed apertures of 10 and $3.3 \mathrm{kpc}$ diameter, focused on the cases of the SDSS and SAMI surveys.

\section{DISCUSSION}

In previous sections we have shown the growth curves of some emission-line fluxes and line ratios, relevant for the estimation of SFRs, extinction, and characteristic oxygen abundances of spiral galaxies. These growth curves are provided with aperture corrections for these quantities that should be considered in a statistical sense, i.e., applicable to large statistical samples of spiral galaxies. The effect of the aperture on $\mathrm{H} \alpha$ luminosity, $\mathrm{H} \alpha / \mathrm{H} \beta$ ratio2, and $\mathrm{O} 3 \mathrm{~N} 2$ is found to be statistically uncorrelated to the inclination, morphological type, and stellar mass of 
Table 21

Ratio of the H $\alpha$ Flux Contained in the SDSS or SAMI Apertures at Different Redshifts to the $\mathrm{H} \alpha$ Flux within a Circular Aperture of 10 or $3.3 \mathrm{kpc}$ Diameter for the CALIFA Spirals Whose 10 or $3.3 \mathrm{kpc}$ Diameter Aperture Is Completely Covered by PMAS/PPAK

\begin{tabular}{lccccc}
\hline \hline $\begin{array}{l}\text { Ang. Diam. } \\
(")\end{array}$ & $\begin{array}{c}\text { Lin. Diam. } \\
(\mathrm{kpc})\end{array}$ & $z$ & $-\sigma$ & $\left(\alpha_{\text {Ang }} / \alpha_{\text {Lin }}\right)_{50}$ & $+\sigma$ \\
\hline 3.0 & 10. & 0.010 & 0.030 & 0.061 & 0.207 \\
3.0 & 10. & 0.011 & 0.030 & 0.060 & 0.206 \\
3.0 & 10. & 0.012 & 0.030 & 0.059 & 0.205 \\
3.0 & 10. & 0.013 & 0.029 & 0.058 & 0.204 \\
3.0 & 10. & 0.014 & 0.029 & 0.057 & 0.204 \\
3.0 & 10. & 0.015 & 0.029 & 0.056 & 0.203 \\
\hline
\end{tabular}

Note. (1) Angular diameter of SDSS/SAMI aperture. (Where the $3^{\prime \prime}$ aperture is for SDSS and the $15^{\prime \prime}$ aperture is for SAMI.) (2) Linear diameter of fixed reference aperture. (3) Redshift. (4)-(6) Values corresponding to $15.86 \%, 50 \%$, and $84.14 \%$ of the distribution. Only the first values are listed.

(This table is available in its entirety in machine-readable form.)

Table 22

Ratio of the $\mathrm{H} \alpha / \mathrm{H} \beta$ Contained in the SDSS or SAMI Apertures at Different Redshifts to the $\mathrm{H} \alpha / \mathrm{H} \beta$ within a Circular Aperture of 10 or $3.3 \mathrm{kpc}$ Diameter for the CALIFA Spirals Whose 10 or $3.3 \mathrm{kpc}$ Diameter Aperture Is Completely Covered by PMAS/PPAK

\begin{tabular}{lccccc}
\hline \hline $\begin{array}{l}\text { Ang. Diam. } \\
\text { (“) }\end{array}$ & $\begin{array}{c}\text { Lin. Diam. } \\
(\mathrm{kpc})\end{array}$ & $z$ & $-\sigma$ & $\left(\alpha \beta_{\text {Ang }} / \alpha \beta_{\text {Lin }}\right)_{50}$ & $+\sigma$ \\
\hline 3.0 & 10. & 0.010 & 0.985 & 1.120 & 1.287 \\
3.0 & 10. & 0.011 & 0.985 & 1.120 & 1.288 \\
3.0 & 10. & 0.012 & 0.985 & 1.120 & 1.288 \\
3.0 & 10. & 0.013 & 0.984 & 1.120 & 1.289 \\
3.0 & 10. & 0.014 & 0.984 & 1.120 & 1.289 \\
3.0 & 10. & 0.015 & 0.984 & 1.120 & 1.290 \\
\hline
\end{tabular}

Note. (1) Angular diameter of SDSS/SAMI aperture. (Where the $3^{\prime \prime}$ aperture is for SDSS and the $15^{\prime \prime}$ aperture is for SAMI.) (2) Linear Diameter of Fixed Reference Aperture. (3) Redshift. (4)-(6) Values Corresponding to $15.86 \%$, $50 \%$, and $84.14 \%$ of the Distribution. Only the First Values are Listed.

(This table is available in its entirety in machine-readable form.)

Table 23

Difference of the N2 Contained in the SDSS or SAMI Apertures at Different Redshifts to the N2 within a Circular Aperture of 10 or $3.3 \mathrm{kpc}$ Diameter for the CALIFA Spirals Whose 10 or $3.3 \mathrm{kpc}$ Diameter Aperture Is Completely Covered by PMAS/PPAK

\begin{tabular}{lccccc}
\hline \hline $\begin{array}{l}\text { Ang. Diam. } \\
(“)\end{array}$ & $\begin{array}{c}\text { Lin. Diam. } \\
(\mathrm{kpc})\end{array}$ & $z$ & $-\sigma$ & $\left(\mathrm{N} 2_{\text {Ang }}-\mathrm{N} 2_{\text {Lin }}\right)_{50}$ & $+\sigma$ \\
\hline 3.0 & 10. & 0.010 & 0.012 & 0.079 & 0.234 \\
3.0 & 10. & 0.011 & 0.011 & 0.079 & 0.234 \\
3.0 & 10. & 0.012 & 0.011 & 0.079 & 0.234 \\
3.0 & 10. & 0.013 & 0.011 & 0.079 & 0.234 \\
3.0 & 10. & 0.014 & 0.011 & 0.079 & 0.234 \\
3.0 & 10. & 0.015 & 0.011 & 0.080 & 0.233 \\
\hline
\end{tabular}

Note. (1) Angular diameter of SDSS/SAMI aperture. (Where the $3^{\prime \prime}$ aperture is for SDSS and the 15" aperture is for SAMI.) (2) Linear Diameter of Fixed Reference Aperture. (3) Redshift. (4)-(6) Values Corresponding to $15.86 \%$, $50 \%$, and $84.14 \%$ of the Distribution. Only the First Values are Listed.

(This table is available in its entirety in machine-readable form.)

galaxies, except for some marginal differences always below the typical dispersions of the median growth curves for small apertures.

\section{Table 24}

Difference of the O3N2 contained in the SDSS or SAMI apertures at Different Redshifts to the O3N2 within a Circular Aperture of 10 or $3.3 \mathrm{kpc}$ Diameter for the CALIFA Spirals Whose 10 or $3.3 \mathrm{kpc}$ Diameter Aperture Is Completely Covered by PMAS/PPAK

\begin{tabular}{lccccc}
\hline \hline $\begin{array}{l}\text { Ang. } \\
\begin{array}{l}\text { Diam. } \\
\left({ }^{\prime \prime}\right)\end{array}\end{array}$ & $\begin{array}{r}\text { Lin. } \\
\text { Diam. } \\
(\mathrm{kpc})\end{array}$ & $z$ & $-\sigma$ & $\begin{array}{c}\left(\mathrm{O} 3 \mathrm{~N} 2_{\text {Ang }}-\right. \\
\left.\mathrm{O} 3 \mathrm{~N} 2_{\text {Lin }}\right)_{50}\end{array}$ & $+\sigma$ \\
\hline 3.0 & 10. & 0.010 & -0.402 & -0.180 & 0.060 \\
3.0 & 10. & 0.011 & -0.402 & -0.180 & 0.060 \\
3.0 & 10. & 0.012 & -0.402 & -0.181 & 0.060 \\
3.0 & 10. & 0.013 & -0.403 & -0.181 & 0.060 \\
3.0 & 10. & 0.014 & -0.403 & -0.181 & 0.060 \\
3.0 & 10. & 0.015 & -0.403 & -0.181 & 0.060 \\
\hline
\end{tabular}

Note. (1) Angular diameter of SDSS/SAMI aperture. (Where the $3^{\prime \prime}$ aperture is for SDSS and the $15^{\prime \prime}$ aperture is for SAMI.) (2) Linear diameter of fixed reference aperture. (3) Redshift. (4)-(6) Values corresponding to $15.86 \%, 50 \%$, and $84.14 \%$ of the distribution. Only the first values are listed.

(This table is available in its entirety in machine-readable form.)

It is interesting to note that the aperture effect found for N2 and $\mathrm{O} 3 \mathrm{~N} 2$ can be interpreted as an aperture effect in the oxygen abundances. As a first step, we illustrate the aperture effect on the oxygen abundances in Figure 19, the median growth curves of the logarithmic oxygen abundance as a function of $R_{50}$, $x\left(\mathrm{OH}_{50}\right),{ }^{24}$ derived using the calibrations by Marino et al. (2013, M13, Pettini \& Pagel (2004, PP04), and Pérez-Montero \& Contini (2009, PMC09), for N2 and O3N2, respectively. As can be seen, the effect of the aperture marginally depends on the calibration used both for $\mathrm{N} 2$ and $\mathrm{O} 3 \mathrm{~N} 2$ showing a maximum difference of $\approx 0.02-0.03 \mathrm{dex}$, which corresponds to $5 \%-7.5 \%$. Although the dispersions around the median values are not shown in the figures (for clarity), they are much lower than this difference.

Figures 20-23 show $x\left(\mathrm{OH}_{50}\right)$ and $x\left(\mathrm{OH}_{\mathrm{eff}}\right)$ estimated from $\mathrm{N} 2$ and $\mathrm{O} 3 \mathrm{~N} 2$ using the M13 calibrations. As shown in the figures, the effect of the aperture for both indicators is maximal for small apertures and only marginal differences (maximum values of $\approx 0.04 \mathrm{dex}$ ) with morphological type and stellar mass are seen, much lower than the typical dispersions around the median values. However, more relevant are the the dispersions around the median values, which can reach maximal values of up to $+25 \%$ for small apertures when deriving oxygen abundances of early-type and/or high-mass spirals using N2. We keep in mind that using other calibrations for the oxygen abundances could result in even larger dispersions around the median values. In particular, as shown in Figure 19, using for example the PMC09 calibrations would result in dispersions $\approx 5 \%$ larger than using the M13 calibrations. This means that when the fluxes are obtained through very small apertures, the aperture-corrected oxygen abundances for individual galaxies could be very uncertain and far from the real values. We stress again that the median aperture corrections for oxygen abundances (and other relevant quantities reported in this work) must be applied to large samples of galaxies and interpreted in a statistical sense.

\footnotetext{
${ }^{24}$ Given that the oxygen abundance is usually a logarithmic quantity, the definition of $x\left(\mathrm{OH}_{50}\right)$ and $x\left(\mathrm{OH}_{\text {eff }}\right)$ at a given radius $r$ is, as in the case of $\mathrm{N} 2$ and $\mathrm{O} 3 \mathrm{~N} 2, \log (\mathrm{O} / \mathrm{H})_{r}-\log (\mathrm{O} / \mathrm{H})_{\text {int }}$, where $\log (\mathrm{O} / \mathrm{H})_{\text {int }}$ corresponds to the value of the logarithmic oxygen abundance measured within the largest aperture considered, $36^{\prime \prime}$, assumed to be the integrated value.
} 

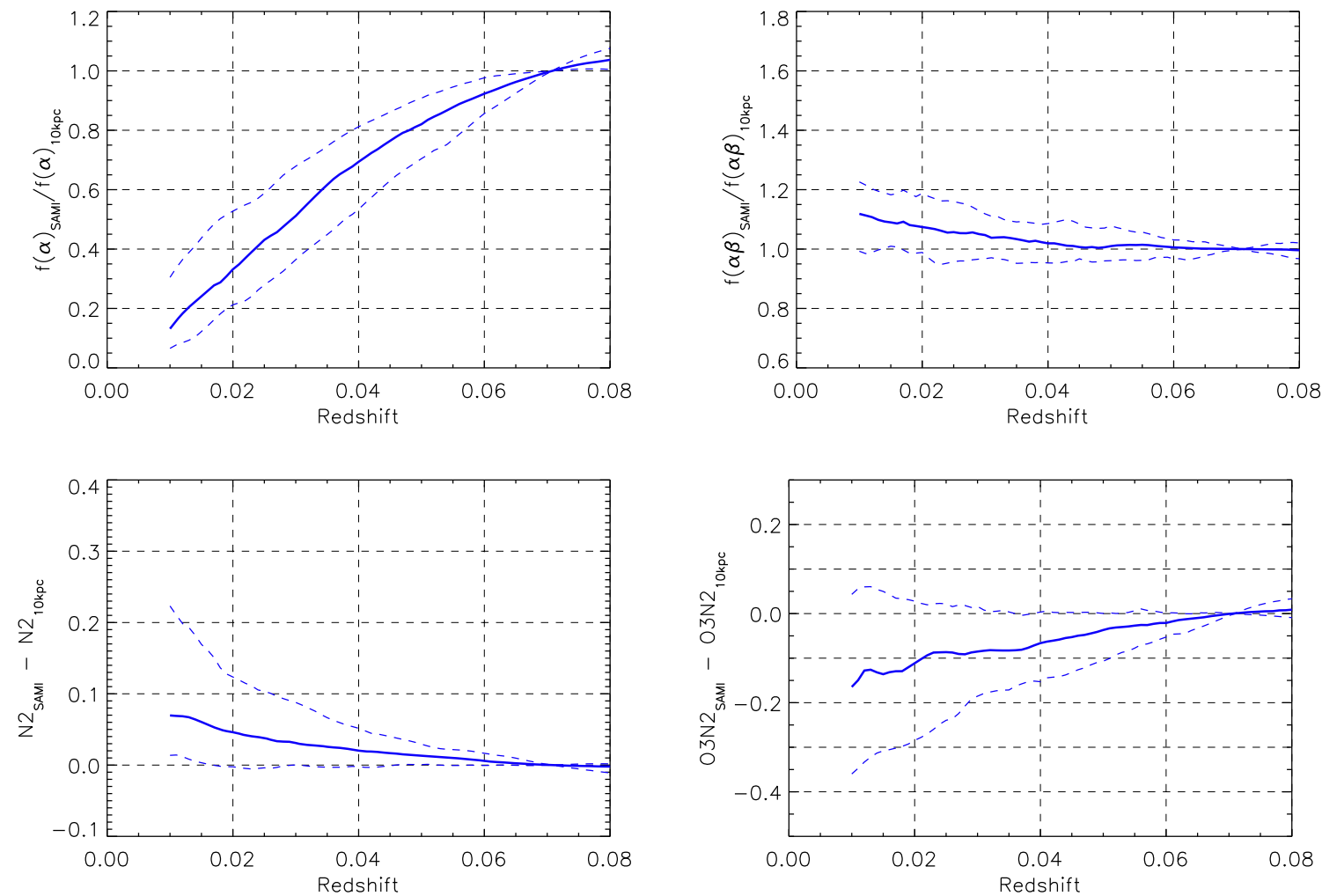

Figure 17. Ratio of the $\mathrm{H} \alpha$ flux (top left), $\mathrm{H} \alpha / \mathrm{H} \beta$ (top right), $\mathrm{N} 2$ (bottom left), and $\mathrm{O} 3 \mathrm{~N} 2$ (bottom right) contained in the SAMI aperture at different redshifts to the corresponding values within a circular aperture of $10 \mathrm{kpc}$ diameter as a function of redshift for the (52) CALIFA spirals whose $10 \mathrm{kpc}$ diameter aperture is completely covered by PMAS/PPAK. Lower dashed, solid, and upper dashed lines correspond to $15.86 \%, 50 \%$, and $84.14 \%$ of the distributions.
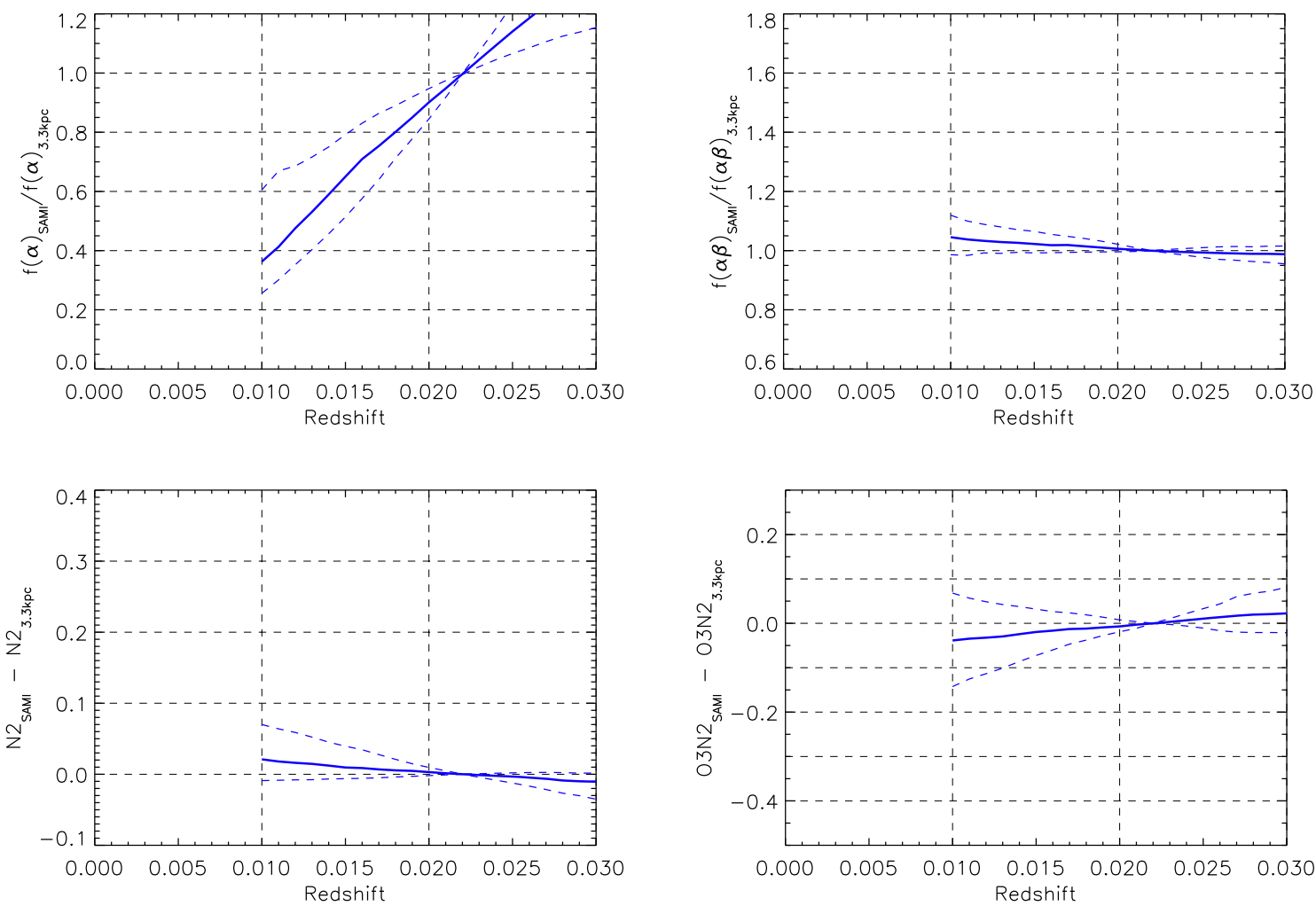

Figure 18. Ratio of the $\mathrm{H} \alpha$ flux (top left), $\mathrm{H} \alpha / \mathrm{H} \beta$ (top right), $\mathrm{N} 2$ (bottom left), and $\mathrm{O} 3 \mathrm{~N} 2$ (bottom right) contained in the SAMI aperture at different redshifts to the corresponding values within a circular aperture of $3.3 \mathrm{kpc}$ diameter as a function of redshift for the (96) CALIFA spirals whose $3.3 \mathrm{kpc}$ diameter aperture is completely covered by PMAS/PPAK. Lower dashed, solid, and upper dashed lines correspond to $15.86 \%, 50 \%$ and $84.14 \%$ of the distributions. 

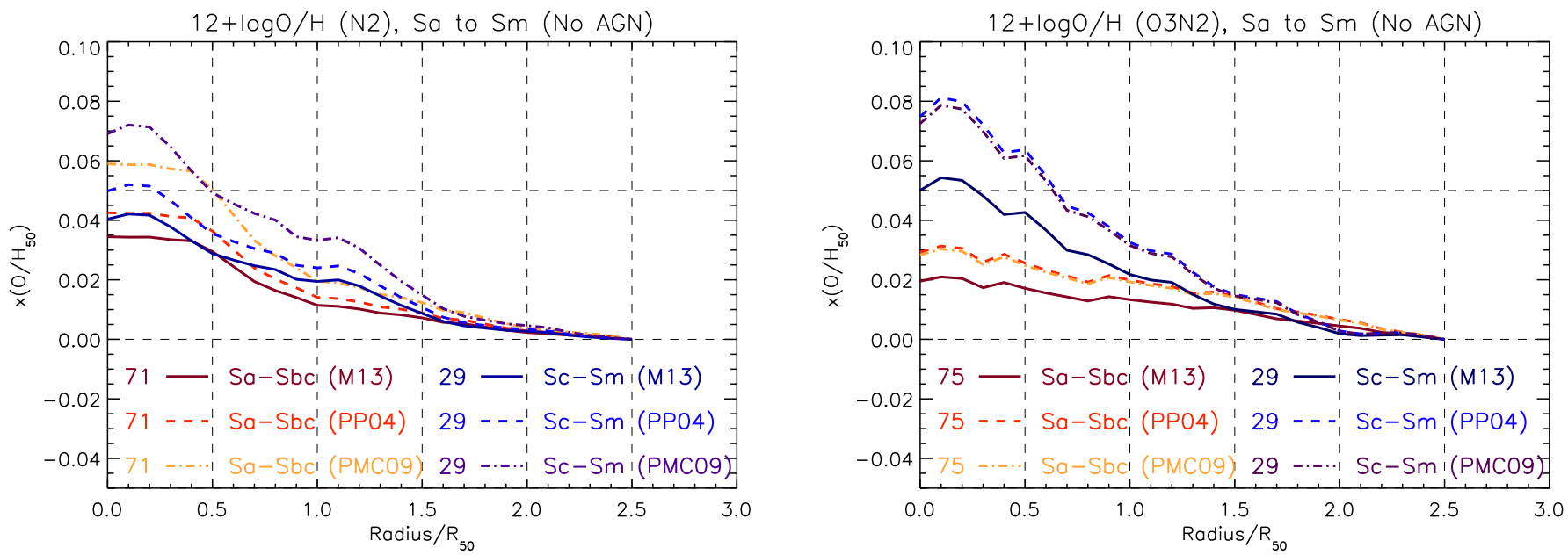

Figure 19. Left: $x\left(\mathrm{OH}_{50}\right)$ for two different morphological type bins, and the three empirical calibrations of $\mathrm{N} 2$ : PP04, PMC09, and M13. Right: $x(\mathrm{OH} 50)$ for two different morphological type bins, and the three empirical calibrations of O3N2: PP04, PMC09, and M13.
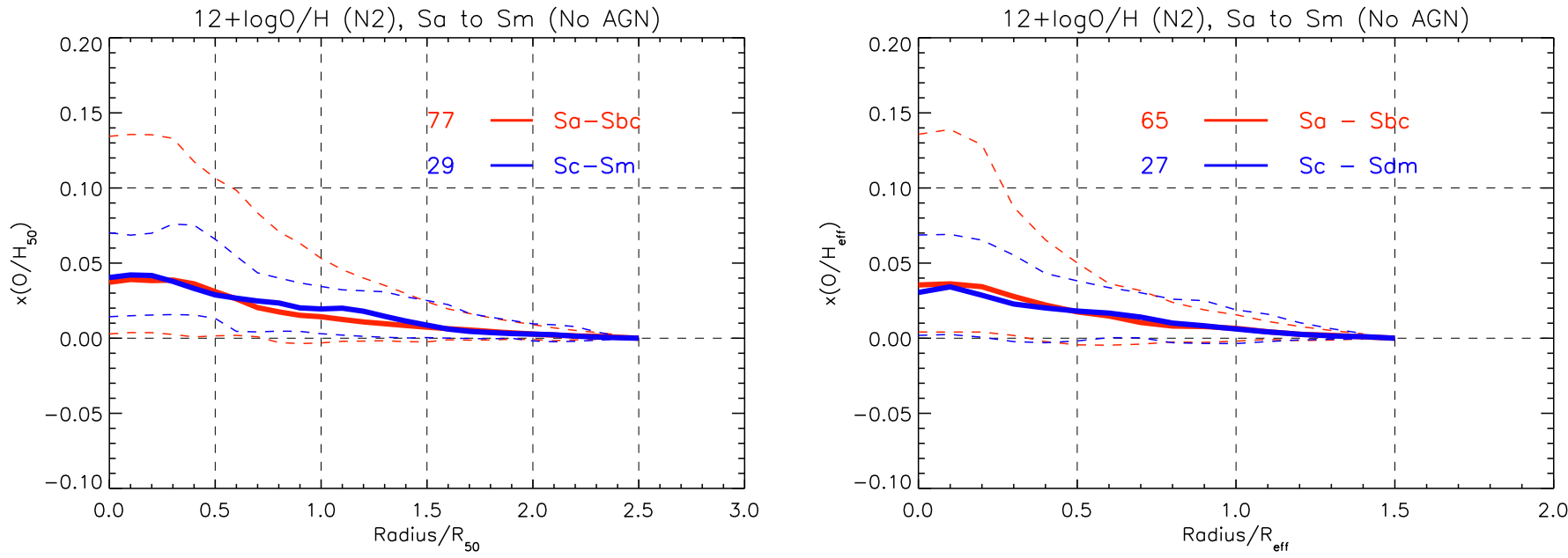

Figure 20. Left: $x\left(\mathrm{OH}_{50}\right)$ for two different morphological type bins, estimated with $\mathrm{N} 2$ (M13). Solid lines correspond to median values. Dashed lines contain $68.2 \%$ of the distribution. Sa-Sbc and Sc-Sm galaxies are represented in blue and red, respectively. Right: same as left for $x\left(\mathrm{OH}_{\text {eff }}\right)$.
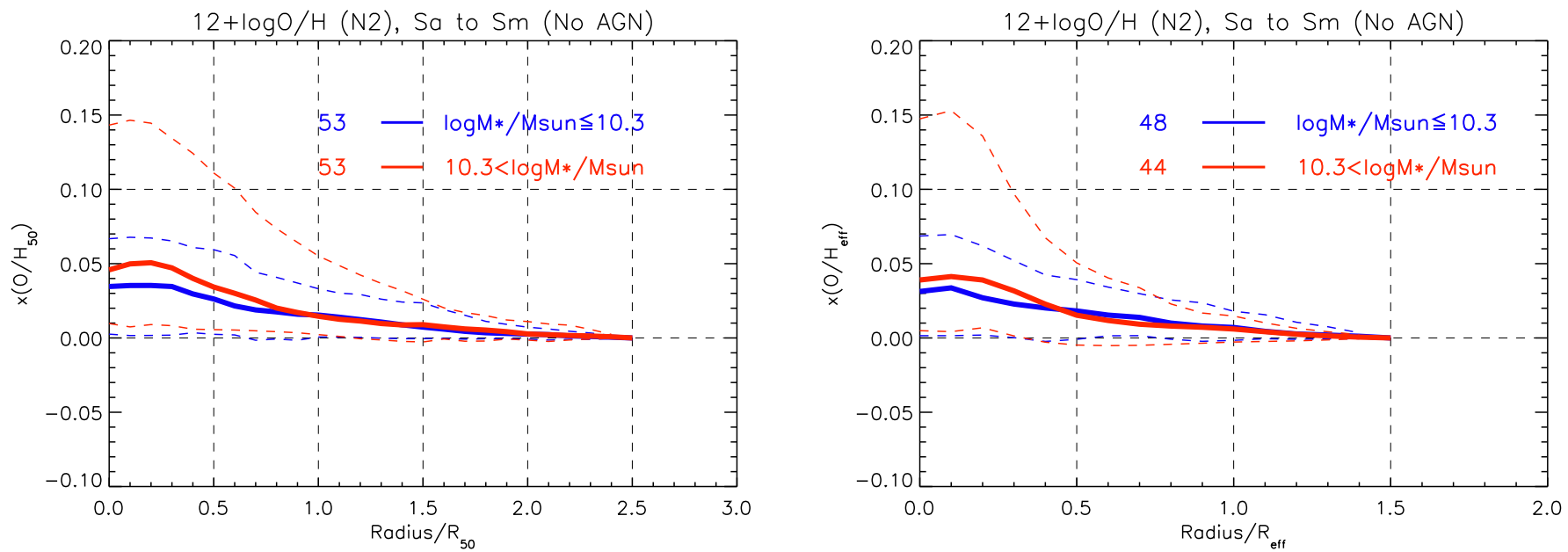

Figure 21. Left: $x\left(\mathrm{OH}_{50}\right)$ for two different stellar mass bins, estimated with $\mathrm{N} 2$ (M13). Solid lines correspond to median values. Dashed lines contain $68.2 \%$ of the distribution. Galaxies with $\log M^{*} / M_{\odot} \leqslant 10.3$ and $\log M^{*} / M_{\odot}>10.3$ are represented in blue and red, respectively. Right: same as left for $x\left(\mathrm{OH}_{\text {eff }}\right)$. 

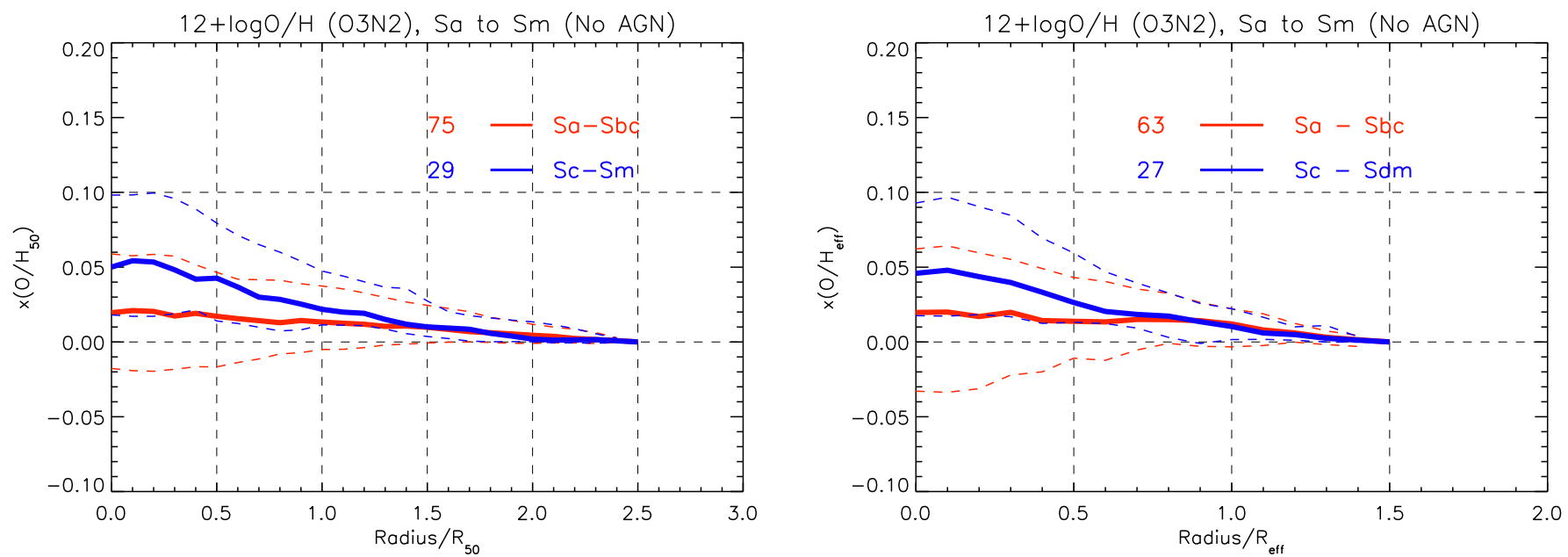

Figure 22. Left: $x\left(\mathrm{OH}_{50}\right)$ for two different morphological type bins, estimated with O3N2 (M13). Solid lines correspond to median values. Dashed lines contain $68.2 \%$ of the distribution. Sa-Sbc and Sc-Sm galaxies are represented in blue and red, respectively. Right: same as left for $x\left(\mathrm{OH}_{\text {eff }}\right)$.
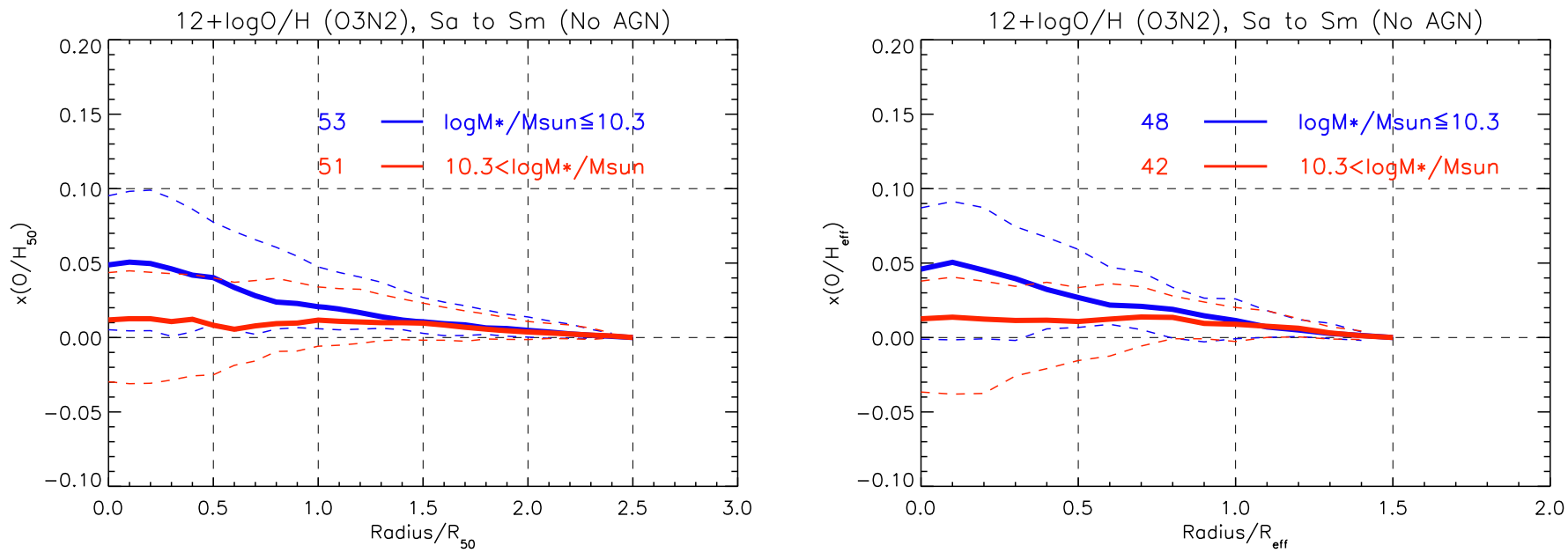

Figure 23. Left: $x\left(\mathrm{OH}_{50}\right)$ for two different stellar mass bins, estimated with O3N2 (M13). Solid lines correspond to median values. Dashed lines contain $68.2 \%$ of the distribution. Galaxies with $\log M^{*} / M_{\odot} \leqslant 10.3$ and $\log M^{*} / M_{\odot}>10.3$ are represented in blue and red, respectively. Right: same as left for $x\left(\mathrm{OH}_{\text {eff }}\right)$.

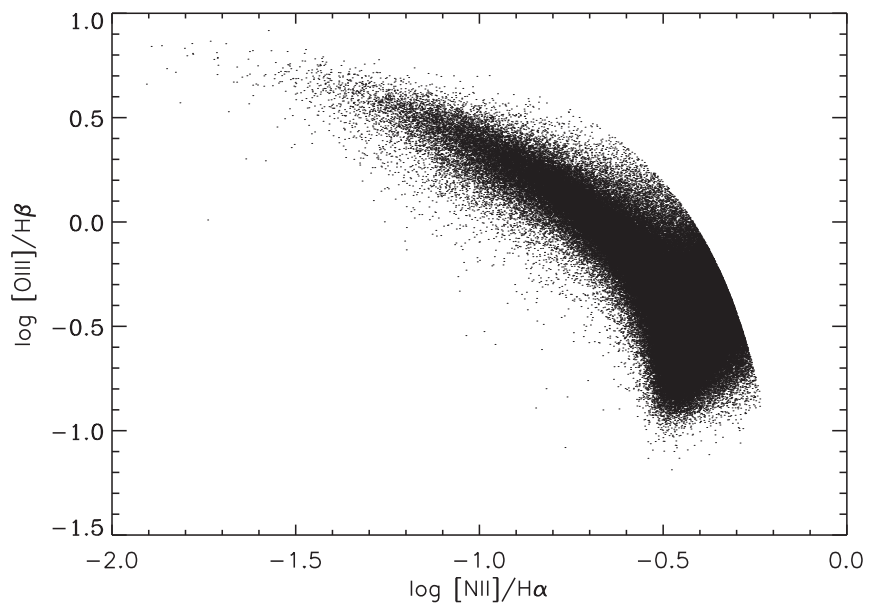

Figure 24. BPT diagram corresponding to the SDSS sample.

\subsection{A Sample Case: SDSS Galaxies at $0.02 \leqslant z \leqslant 0.3$}

In this subsection we apply the results of our work on a sample of galaxies from the SDSS survey and quantify the average effect of the aperture resulting when estimating the oxygen abundance from the flux measured through the SDSS fiber. For this we will make use of the corrections derived using $R_{50}$ and split in two bins of stellar masses, since these two parameters are available for all the SDSS galaxies.

The sample of galaxies is the MPA-JHU sample (Kauffmann et al. 2003; Brinchmann et al. 2004; Tremonti et al. 2004, and Salim et al. 2007), which provides stellar masses and uses spectroscopy from SDSS-DR7 (Abazajian et al. 2009), and complementary photometry from SDSS-DR12 (Alam et al. 2015), satisfying the following criteria:

1. Redshift in the range $0.02 \leqslant z \leqslant 0.3$.

2. Signal-to-noise ratio of the emission lines $\mathrm{H} \alpha, \mathrm{H} \beta$, [N II] $\lambda 6583$, and [O III] $\lambda 5007$ larger than 3 .

3. Stellar mass in the range $8.5 \leqslant \log M^{*} / M_{\odot} \leqslant 11.5$

4. Signal-to-noise ratio of the half-light Petrosian radius in the $r^{\prime}$ band $\left(R_{50}\right)$ larger than 3 .

Next we remove those galaxies classified as QSOs by SDSS. We also remove those galaxies not classified as star-forming galaxies according to the BPT diagram (Baldwin et al. 1981) of 

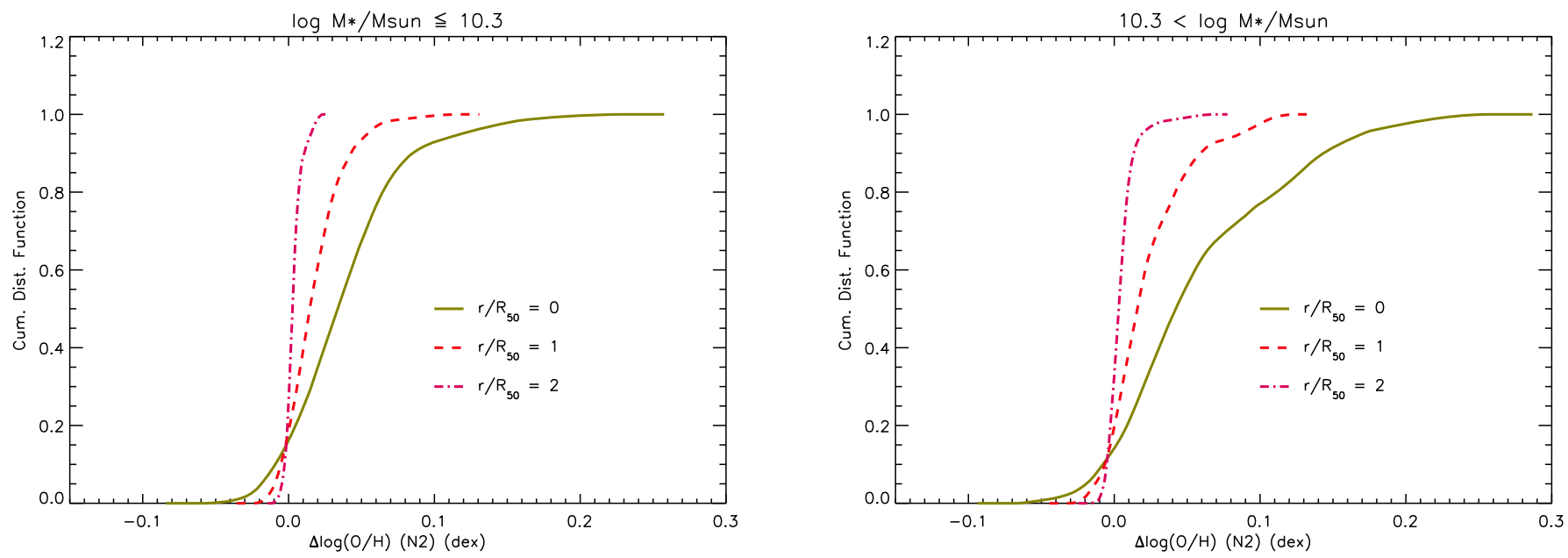

Figure 25. Left: cumulative distribution function (CDF) of $x\left(\mathrm{OH}_{50}\right)$ estimated with $\mathrm{N} 2$, for galaxies with $\log M^{*} / M_{\odot} \leqslant 10.3$ for apertures of three different radii. Right: cumulative distribution function (CDF) of $x\left(\mathrm{OH}_{50}\right)$ estimated with $\mathrm{N} 2$, for galaxies with $\log M^{*} / M_{\odot}>10.3$ for apertures of three different radii.
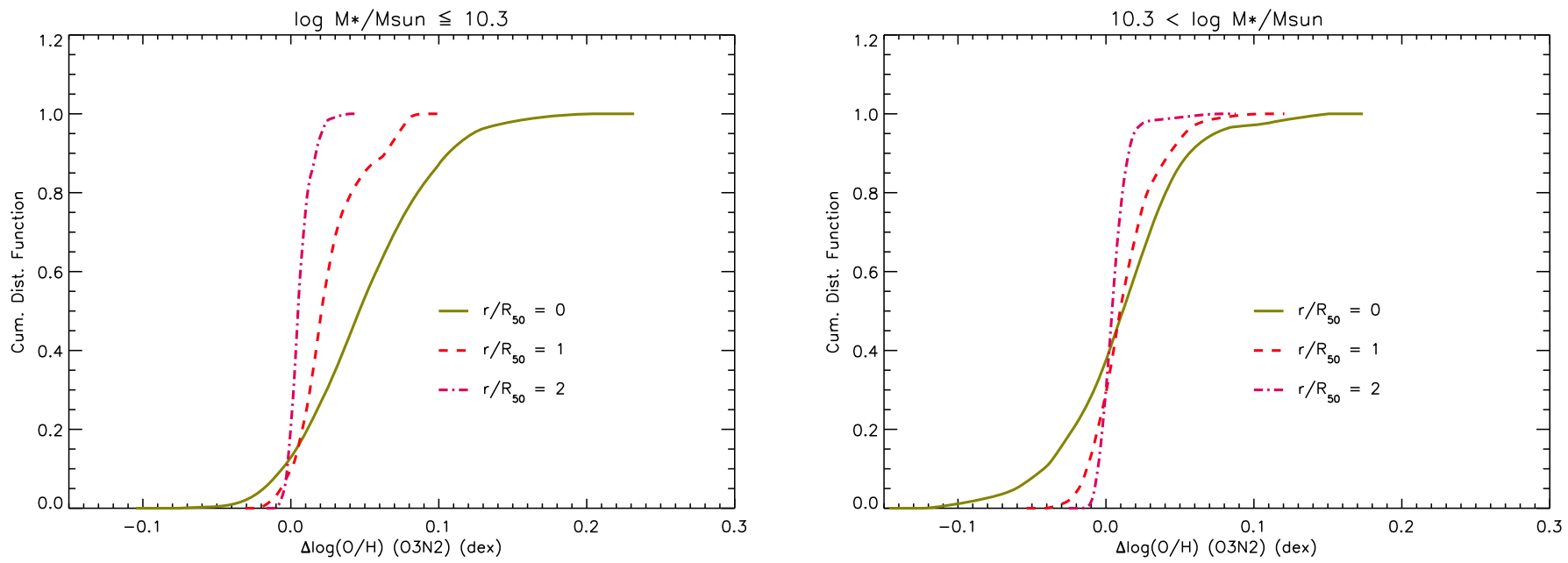

Figure 26. Left: cumulative distribution function (CDF) of $x\left(\mathrm{OH}_{50}\right)$ estimated with $\mathrm{O} 3 \mathrm{~N} 2$, for galaxies with $\log M^{*} / M_{\odot} \leqslant 10.3$ for apertures of three different radii. Right: cumulative distribution function (CDF) of $x\left(\mathrm{OH}_{50}\right)$ estimated with $\mathrm{O} 3 \mathrm{~N} 2$, for galaxies with $\log M^{*} / M_{\odot}>10.3$ for apertures of three different radii.

Kauffmann et al. (2003) and the condition $\operatorname{EW}(\mathrm{H} \alpha) \geqslant 3 \AA$ as indicated in the WHAN diagram of Cid Fernandes et al. (2011). Finally, we keep only the galaxies for which $0.3 \leqslant 1$ " $5 / R_{50} \leqslant$ 2.5 , as it is the range of applicability of the corrections as indicated in IP13. Figure 24 shows the BPT diagram of the SDSS sample.

In order to be as realistic as possible, we want to take into account the large dispersion observed around the median values of the growth curves for small apertures. For this, we first construct approximate cumulative distribution functions (CDFs) of the distribution of the individual $\mathrm{O} / \mathrm{H}$ growth curves at each radius for the two stellar mass bins previously considered in Figures 21 and 23. Figures 25 and 26 show the CDFs at three different radii normalized to $R_{50}$. As can be seen, the larger the radius normalized with respect to $R_{50}$, the closer the distribution remains to zero, as we observed in the previous section. Several Monte Carlo tests were produced to verify that the random distributions following these CDFs are consistent with being drawn from the same parental distribution as the original distributions from the CALIFA galaxies.
Then we are ready to quantify the aperture effect with a sample of galaxies from SDSS for which the stellar mass, $R_{50}$, and the fluxes of the required emission lines are well known. To each of the galaxies we will apply a value of the correction corresponding to its stellar mass and coverage of the SDSS fiber (normalized to $R_{50}$ ) taken at random from the corresponding CDF. The difference between the oxygen abundances estimated from the flux of the SDSS fiber and that we obtained using the aperture correction as described above was computed for each galaxy using the two indicators $\mathrm{N} 2$ and $\mathrm{O} 3 \mathrm{~N} 2$. Then, the median value of these differences was computed for five stellar mass bins: $\log M^{*} / M_{\odot} \in[8.5,9.1],[9.1,9.7]$, [9.7, 10.3], [10.3, 10.9], and [10.9, 11.5]. We repeated this process a total of 25 times, and computed the average value of the median of the differences for each of these four mass bins. Table 25 shows the results of this simulation split in six redshift bins: $z \in[0.02,0.05],[0.05,0.10],[0.10,0.15],[0.15,0.20]$, $[0.20,0.25]$, and $[0.25,0.30]$.

In this study the aperture effect depends on two different aspects: on the one side, the fraction of galaxy covered by the 
Table 25

Differential (Fiber-based vs. Integrated) Oxygen Abundance of the SDSS Galaxies Estimated with the N2 and O3N2 Indicators at Three Redshift Bins

\begin{tabular}{|c|c|c|c|}
\hline & $N_{\text {gal }}$ & $\begin{array}{c}\langle\operatorname{Median}(\Delta \\
\underset{(\operatorname{dex})}{\left.\left.\log O / H_{\mathrm{N} 2}\right)\right\rangle}\end{array}$ & $\begin{array}{r}\langle\operatorname{Median}(\Delta \\
\left.\left.\log \mathrm{O} / \mathrm{H}_{\mathrm{O} 3 \mathrm{~N} 2}\right)\right\rangle \\
(\mathrm{dex})\end{array}$ \\
\hline \multicolumn{4}{|c|}{$0.02<z<0.05$} \\
\hline $8.5 \leqslant \log M^{*} / M_{\odot}<9.1$ & 10640 & 0.025 & 0.034 \\
\hline $9.1 \leqslant \log M^{*} / M_{\odot}<9.7$ & 16964 & 0.026 & 0.035 \\
\hline $9.7 \leqslant \log M^{*} / M_{\odot}<10.3$ & 8817 & 0.027 & 0.037 \\
\hline $10.3 \leqslant \log M^{*} / M_{\odot}<10.9$ & 1698 & 0.045 & 0.013 \\
\hline $10.9 \leqslant \log M^{*} / M_{\odot}<11.5$ & 44 & 0.047 & 0.013 \\
\hline \multicolumn{4}{|c|}{$0.05<z<0.10$} \\
\hline $8.5 \leqslant \log M^{*} / M_{\odot}<9.1$ & 994 & 0.015 & 0.020 \\
\hline $9.1 \leqslant \log M^{*} / M_{\odot}<9.7$ & 17560 & 0.022 & 0.029 \\
\hline $9.7 \leqslant \log M^{*} / M_{\odot}<10.3$ & 48676 & 0.023 & 0.031 \\
\hline $10.3 \leqslant \log M^{*} / M_{\odot}<10.9$ & 24456 & 0.036 & 0.012 \\
\hline $10.9 \leqslant \log M^{*} / M_{\odot}<11.5$ & 1348 & 0.042 & 0.013 \\
\hline \multicolumn{4}{|c|}{$0.10<z<0.15$} \\
\hline $8.5 \leqslant \log M^{*} / M_{\odot}<9.1$ & 21 & 0.012 & 0.017 \\
\hline $9.1 \leqslant \log M^{*} / M_{\odot}<9.7$ & 1140 & 0.012 & 0.017 \\
\hline $9.7 \leqslant \log M^{*} / M_{\odot}<10.3$ & 12599 & 0.019 & 0.025 \\
\hline $10.3 \leqslant \log M^{*} / M_{\odot}<10.9$ & 29146 & 0.030 & 0.011 \\
\hline $10.9 \leqslant \log M^{*} / M_{\odot}<11.5$ & 3748 & 0.034 & 0.012 \\
\hline \multicolumn{4}{|c|}{$0.15<z<0.20$} \\
\hline $8.5 \leqslant \log M^{*} / M_{\odot}<9.1$ & 0 & $\cdots$ & $\cdots$ \\
\hline $9.1 \leqslant \log M^{*} / M_{\odot}<9.7$ & 135 & 0.010 & 0.014 \\
\hline $9.7 \leqslant \log M^{*} / M_{\odot}<10.3$ & 1972 & 0.013 & 0.017 \\
\hline $10.3 \leqslant \log M^{*} / M_{\odot}<10.9$ & 7540 & 0.026 & 0.011 \\
\hline $10.9 \leqslant \log M^{*} / M_{\odot}<11.5$ & 3445 & 0.030 & 0.012 \\
\hline \multicolumn{4}{|c|}{$0.20<z<0.25$} \\
\hline $8.5 \leqslant \log M^{*} / M_{\odot}<9.1$ & 1 & $\cdots$ & $\cdots$ \\
\hline $9.1 \leqslant \log M^{*} / M_{\odot}<9.7$ & 20 & 0.008 & 0.012 \\
\hline $9.7 \leqslant \log M^{*} / M_{\odot}<10.3$ & 494 & 0.011 & 0.015 \\
\hline $10.3 \leqslant \log M^{*} / M_{\odot}<10.9$ & 1710 & 0.018 & 0.011 \\
\hline $10.9 \leqslant \log M^{*} / M_{\odot}<11.5$ & 1698 & 0.028 & 0.011 \\
\hline \multicolumn{4}{|c|}{$0.25<z<0.30$} \\
\hline $8.5 \leqslant \log M^{*} / M_{\odot}<9.1$ & 0 & $\cdots$ & $\cdots$ \\
\hline $9.1 \leqslant \log M^{*} / M_{\odot}<9.7$ & 1 & $\cdots$ & $\cdots$ \\
\hline $9.7 \leqslant \log M^{*} / M_{\odot}<10.3$ & 43 & 0.009 & 0.013 \\
\hline $10.3 \leqslant \log M^{*} / M_{\odot}<10.9$ & 493 & 0.014 & 0.011 \\
\hline $10.9 \leqslant \log M^{*} / M_{\odot}<11.5$ & 357 & 0.022 & 0.011 \\
\hline
\end{tabular}

Note. (1) Stellar mass bin; (2) number of galaxies; (3) average over 25 random draws of the median values of $\log \mathrm{O} / \mathrm{H}_{\text {SDSS }}-\log \mathrm{O} / \mathrm{H}_{\text {int }}$ estimated using $\mathrm{N} 2$; (4) average over 25 random draws of the median values of $\log \mathrm{O} /$ $\mathrm{H}_{\text {SDSS }}-\log \mathrm{O} / \mathrm{H}_{\text {int }}$ estimated using O3N2.

SDSS fiber is partly driven by the redshift of the galaxies. On the other hand, the aperture effect depends on the stellar mass of the galaxy. Thus, Table 25 must show a combination of these effects. The first thing we note is that the maximum average aperture effect measured with these two calibrations at the redshifts and stellar mass ranges probed is of the order of $\approx 0.047 \mathrm{dex}(\approx 11 \%)$, which as we previously said is much smaller than the typical uncertainties of any of the two empirical calibrations. The average corrections when using the $\mathrm{N} 2$ calibration tend to increase with stellar mass at all redshift ranges probed, and for a given stellar mass, the average correction decreases when redshift increases. Regarding the O3N2 calibration, it reaches a maximum for stellar masses $9.7<\log M^{*} / M_{\odot}<10.3$ at all redshift ranges, and for a given mass range it tends to decrease as redshift increases.

We also remark that in this work we have studied the effect of the aperture on the indicators used to estimate the oxygen abundance (N2 and O3N2). However, it is out of the scope of this paper to enter into the discussion about the goodness of the integrated values of these two indicators as proxies for the oxygen abundance, since they contain emission not only from star-forming regions but also from the diffuse ionized gas of the disks of spiral galaxies. It has been reported in the literature that the oxygen abundance estimated from integrated fluxes of emission lines approximately equals the corresponding abundance of star-forming regions at the galactocentric distance $0.4 \times R_{\text {opt }}$ (Pilyugin et al. 2004; Moustakas \& Kennicutt 2006). Although this relation links these two quantities, further information is still required in order to get information about the chemical evolution of spiral galaxies.

\section{CONCLUSIONS}

This paper presents the growth curves taken through circular apertures of several emission-line fluxes for a sample of spiral galaxies from the CALIFA project. These curves allow us to study the effect of estimating galactic properties from the light of a circular aperture only partially covering the spatial extent of a galaxy instead of using the integrated light. The main conclusions arising from this work are the following:

1. Whereas the median $\mathrm{H} \alpha$ growth curves are insensitive to inclination, morphology, and total stellar mass of the studied galaxies, our analysis documents a strong dependence of the registered $\mathrm{H} \alpha$ luminosity on the aperture size (and consequently, also on redshift).

2. The median $\mathrm{H} \alpha / \mathrm{H} \beta$ growth curves are insensitive to the inclination, morphological types, and stellar masses of the galaxies, and shows a mild trend with a maximum for small apertures smoothly decreasing toward large apertures, which means that the extinction is overestimated on average when observing galaxies through small apertures.

3. The median $\mathrm{N} 2$ growth curves are also insensitive to the inclinations, morphological types, and stellar masses of the galaxies, showing a decreasing trend toward large apertures. This means that the oxygen abundance is overestimated when observed through small apertures.

4. The median $\mathrm{O} 3 \mathrm{~N} 2$ growth curves are insensitive to the inclinations, and show higher values for galaxies of earlier morphological types and $\log M^{*} / M_{\odot}>10.3$ at small radii apertures. Again, this means that estimating oxygen abundances through small apertures tends to overestimate the abundances.

5. When applying our aperture corrections to a sample of SDSS galaxies with $0.02 \leqslant z \leqslant 0.3$, the average corrected oxygen abundances derived from the SDSS fluxes may as a result be enhanced by a maximum of $\approx 11 \%$ with respect to the fiber-based ones.

This work has shown that although the median aperture corrections for oxygen abundance are always small, the dispersions around these median values become very large for small apertures, thus preventing the use of aperture corrections for studies of individual galaxies unless a very large uncertainty is assumed. A more detailed study of the 
aperture effects on some individual emission-line ratios will be presented in a forthcoming paper.

This study made use of the data provided by the Calar Alto Legacy Integral Field Area (CALIFA) survey (http://califa.caha. es/). The CALIFA collaboration would like to thank the IAACSIC and MPIA-MPG as major partners of the observatory, and CAHA itself, for unique access to telescope time and support in manpower and infrastructure. The CALIFA collaboration also thanks the CAHA staff for the dedication to this project. Based on observations collected at the Centro Astronómico Hispano Alemán (CAHA) at Calar Alto, operated jointly by the MaxPlanck-Institut für Astronomie and the Instituto de Astrofísica de Andalucía (CSIC). We thank the Viabilidad, Diseño, Acceso y Mejora funding program ICTS-2009-10, for supporting the initial developement of this project. J.I.P., J.V.M., C.K., E.P.M., and S. D.P. acknowledge financial support from the Spanish MINECO under grant AYA2010-21887-C04-01, and from Junta de Andalucía Excellence Project PEX2011-FQM7058. C.C.T. and A.C.M. also thank the support from the Plan Nacional de Investigación y Desarrollo funding program AYA2013-46724-P. J.M.G. acknowledges support by Fundação para a Ciência e a Tecnologia (FCT) through the Fellowship SFRH/BPD/66958/ 2009 and POPH/FSE (EC) by FEDER funding through the program Programa Operacional de Factores de Competitividade (COMPETE). P.P. is supported by FCT through the Investigador FCT Contract No. IF/01220/2013 and POPH/FSE (EC) by FEDER funding through the program COMPETE. J.M.G. and P. P. also acknowledge support by FCT under project FCOMP-010124-FEDER-029170 (Reference FCT PTDC/FIS-AST/3214/ 2012), funded by FCT-MEC (PIDDAC) and FEDER (COM PETE). They also acknowledge support by the exchange programme Study of Emission-Line Galaxies with Integral-Field Spectroscopy (SELGIFS, FP7-PEOPLE-2013-IRSES-612701), funded by the EU through the IRSES scheme. F.F.R.O. acknowledges the exchange programme Study of Emission-Line Galaxies with Integral-Field Spectroscopy(SELGIFS, FP7-PEO PLE-2013-IRSES-612701), funded by the EU through the IRSES scheme. Support for L.G. is provided by the Ministry of Economy, Development, and Tourism's Millennium Science Initiative through grant IC120009, awarded to The Millennium Institute of Astrophysics, MAS. L.G. acknowledges support by CONICYT through FONDECYT grant 3140566. This research has made use of the NASA/IPAC Extragalactic Database (NED) which is operated by the Jet Propulsion Laboratory, California Institute of Technology, under contract with the National Aeronautics and Space Administration. We acknowledge the usage of the HyperLeda database (http://leda.univ-lyon1.fr). Funding for SDSS-III has been provided by the Alfred P. Sloan Foundation, the Participating Institutions, the National Science Foundation, and the U.S. Department of Energy Office of Science. SDSS-III is managed by the Astrophysical Research Consortium for the Participating Institutions of the SDSS-III Collaboration including the University of Arizona, the Brazilian Participation Group, Brookhaven National Laboratory, Carnegie Mellon University, University of Florida, the French Participation Group, the German Participation Group, Harvard University, the Instituto de Astrofísica de Canarias, the Michigan State/Notre Dame/JINA Participation Group, Johns Hopkins University, Lawrence Berkeley National Laboratory, Max Planck Institute for Astrophysics, Max Planck Institute for Extraterrestrial Physics, New Mexico State University, New York University, Ohio State
University, Pennsylvania State University, University of Ports mouth, Princeton University, the Spanish Participation Group, University of Tokyo, University of Utah, Vanderbilt University, University of Virginia, University of Washington, and Yale University. The SDSS-III Web site is http://www.sdss3.org.

\section{REFERENCES}

Abazajian, K. N., Adelman-McCarthy, J. K., Agüeros, M. A., et al. 2009, ApJS, 182, 543

Alam, S., Albareti, F. D., Allende Prieto, C., et al. 2015, ApJS, 219, 12

Allen, J. T., Croom, S. M., Konstantopoulos, I. S., et al. 2015, MNRAS, 446, 1567

Bacon, R., Copin, Y., Monnet, G., et al. 2001, MNRAS, 326, 23

Baldwin, J. A., Phillips, M. M., \& Terlevich, R. 1981, PASP, 93, 5

Best, P. N., \& Heckman, T. M. 2012, MNRAS, 421, 1569

Blanc, G. A., Gebhardt, K., Heiderman, A., et al. 2010, in ASP Conf. Ser. 432, New Horizons in Astronomy: Frank N. Bash Symp. 2009 ed. L. M. Stanford et al. (San Francisco, CA: ASP), 180

Brinchmann, J., Charlot, S., White, S. D. M., et al. 2004, MNRAS, 351, 1151

Bryant, J. J., Owers, M. S., Robotham, A. S. G., et al. 2015, MNRAS, 447, 2857

Cappellari, M., Emsellem, E., Krajnović, D., et al. 2011, MNRAS, 413, 813

Cid Fernandes, R., Pérez, E., García Benito, R., et al. 2013, A\&A, 557, A86

Cid Fernandes, R., Stasińska, G., Mateus, A., \& Vale Asari, N. 2011, MNRAS, 413, 1687

Colless, M., Dalton, G., Maddox, S., et al. 2001, MNRAS, 328, 1039

Davis, M., Faber, S. M., Newman, J., et al. 2003, Proc. SPIE, 4834, 161

Ellis, S. C., Driver, S. P., Allen, P. D., et al. 2005, MNRAS, 363, 1257

García-Benito, R., Zibetti, S., Sánchez, S. F., et al. 2015, A\&A, 576, A135

Gerssen, J., Wilman, D. J., \& Christensen, L. 2012, MNRAS, 420, 197

Gomes, J. M., Papaderos, P., Kehrig, C., et al. 2015a, arXiv:1511.02191

Gomes, J. M., Papaderos, P., Vílchez, J. M., et al. 2015b, arXiv:1511.01300

González Delgado, R. M., Pérez, E., Cid Fernandes, R., et al. 2014, A\&A, 562, A47

Heiderman, A. L., Evans, N. J., II, Gebhardt, K., et al. 2011, in New Horizons in Astronomy, Proc. of the Frank N. Bash Symp. 2011, ed. S. Salviander, J. Green, \& A. Pawlik, http://pos.sissa.it/cgi-bin/reader/conf.cgi? confid $=149$

Hopkins, A. M., Miller, C. J., Nichol, R. C., et al. 2003, ApJ, 599, 971

Iglesias-Páramo, J., Vílchez, J. M., Galbany, L., et al. 2013, A\&A, 553, L7

Kauffmann, G., Heckman, T. M., Tremonti, C., et al. 2003, MNRAS, 346, 1055

Kehrig, C., Monreal-Ibero, A., Papaderos, P., et al. 2012, A\&A, 540, A11

Kehrig, C., Pérez-Montero, E., Vílchez, J. M., et al. 2013, MNRAS, 432, 2731

Kewley, L. J., Dopita, M. A., Sutherland, R. S., Heisler, C. A., \& Trevena, J. 2001, ApJ, 556, 121

Kewley, L. J., \& Ellison, S. L. 2008, ApJ, 681, 1183

Kewley, L. J., Groves, B., Kauffmann, G., \& Heckman, T. 2006, MNRAS, 372, 961

Kewley, L. J., Jansen, R. A., \& Geller, M. J. 2005, PASP, 117, 227

Kroupa, P. 2001, MNRAS, 322, 231

Le Fèvre, O., Vettolani, G., Garilli, B., et al. 2005, A\&A, 439, 845

Lilly, S. J., Le Fèvre, O., Renzini, A., et al. 2007, ApJS, 172, 70

Makarov, D., Prugniel, P., Terekhova, N., Courtois, H., \& Vauglin, I. 2014, A\&A, 570, A13

Marino, R. A., Rosales-Ortega, F. F., Sánchez, S. F., et al. 2013, A\&A, 559, A114

Mast, D., Rosales-Ortega, F. F., Sánchez, S. F., et al. 2014, A\&A, 561, A129

Moustakas, J., \& Kennicutt, R. C., Jr. 2006, ApJ, 651, 155

Papaderos, P., Gomes, J. M., Vílchez, J. M., et al. 2013, A\&A, 555, L1

Pérez-Montero, E., \& Contini, T. 2009, MNRAS, 398, 949

Pettini, M., \& Pagel, B. E. J. 2004, MNRAS, 348, L59

Pilyugin, L. S., Contini, T., \& Vílchez, J. M. 2004, A\&A, 423, 427

Richards, S. N., Bryant, J., Croom, S., et al. 2015, arXiv:1510.06038

Rosales-Ortega, F. F., Kennicutt, R. C., Sánchez, S. F., et al. 2010, MNRAS, 405, 735

Roth, M. M., Kelz, A., Fechner, T., et al. 2005, PASP, 117, 620

Salim, S., Rich, R. M., Charlot, S., et al. 2007, ApJS, 173, 267

Sánchez, S. F., Kennicutt, R. C., Gil de Paz, A., et al. 2012, A\&A, 538, A8

Sánchez, S. F., Rosales-Ortega, F. F., Iglesias-Páramo, J., et al. 2014, A\&A, 563, A49

Sánchez, S. F., Rosales-Ortega, F. F., Jungwiert, B., et al. 2013, A\&A, 554, A58

Sánchez-Blázquez, P., Peletier, R. F., Jiménez-Vicente, J., et al. 2006, MNRAS, 371, 703

Sharp, R., Allen, J. T., Fogarty, L. M. R., et al. 2015, MNRAS, 446, 1551 
Singh, R., van de Ven, G., Jahnke, K., et al. 2013, A\&A, 558, A43

Tremonti, C. A., Heckman, T. M., Kauffmann, G., et al. 2004, ApJ, 613, 898

Vazdekis, A., Sánchez-Blázquez, P., Falcón-Barroso, J., et al. 2010, MNRAS, 404, 1639
Walcher, C. J., Wisotzki, L., Bekeraité, S., et al. 2014, A\&A, 569, A1

York, D. G., Adelman, J., Anderson, J. E., Jr., et al. 2000, AJ, 120, 1579

Zahid, H. J., Yates, R. M., Kewley, L. J., \& Kudritzki, R. P. 2013, ApJ, 763,92 